\title{
Accurate assessment of amino acid mass isotopomer distributions for metabolic flux analysis
}

\section{Supporting Information}

Maciek R. Antoniewicz, Joanne K. Kelleher and Gregory Stephanopoulos

Department of Chemical Engineering, Bioinformatics and Metabolic Engineering

Laboratory, Massachusetts Institute of Technology, Cambridge MA 02139, USA

\begin{abstract}
This document provides the acquired mass isotopomer distributions of natural and [U-

$\left.{ }^{13} \mathrm{C}\right]$ enriched amino acids. Amino acid standards were derivatized to their respective TBDMS derivatives and analyzed by GC-EI-MS. Samples were analyzed 35 times at varying concentrations. Mass isotopomer distributions were obtained by integration and corrected for the concentration effect as described in the primary article.
\end{abstract}




\section{Materials and Methods}

GC/MS analysis of unlabeled and $\left[\mathrm{U}-{ }^{13} \mathrm{C}\right]-$ labeled (99 At $\%$ ) amino acids was performed on a HP 5890 Series II GC connected to HP 5971 MSD. The mass spectrometer was calibrated using the 'Max Sensitivity Autotune' setting.

\section{Column Specifications}

- $\quad$ Agilent Technologies 122-0732 DB-1701

- Length: $30 \mathrm{~m}$

- ID: $0.25 \mathrm{~mm}$

- Film: $0.25 \mu \mathrm{m}$

- Temperature limits: $-20^{\circ} \mathrm{C}$ to $280^{\circ} \mathrm{C}$

\section{MSD Settings}

- Scan mode

- 7 min solvent delay

- 23 min recording time

- $\quad$ Mass range: $\mathrm{m} / \mathrm{z} 195$ to 445 (approx. 2.7 scans/sec)

\section{GC Settings}

- Constant flow $0.737 \mathrm{ml} / \mathrm{min}\left(=6\right.$ psi at $\left.80^{\circ} \mathrm{C} ; 31.6 \mathrm{~cm} / \mathrm{sec}\right)$

- Injector temp: $270^{\circ} \mathrm{C}$

- Transfer line temp: $300^{\circ} \mathrm{C}$

- Temperature program:
o $100^{\circ} \mathrm{C}$ (hold $1.5 \mathrm{~min}$ )
o $20^{\circ} \mathrm{C} / \mathrm{min}$ to $130^{\circ} \mathrm{C}$
o $10^{\circ} \mathrm{C} / \mathrm{min}$ to $220^{\circ} \mathrm{C}$ (hold $3 \mathrm{~min}$ )
o $5^{\circ} \mathrm{C} / \mathrm{min}$ to $280^{\circ} \mathrm{C}$ (hold $3 \mathrm{~min}$ ) 


\section{Total Ion Chromatogram}
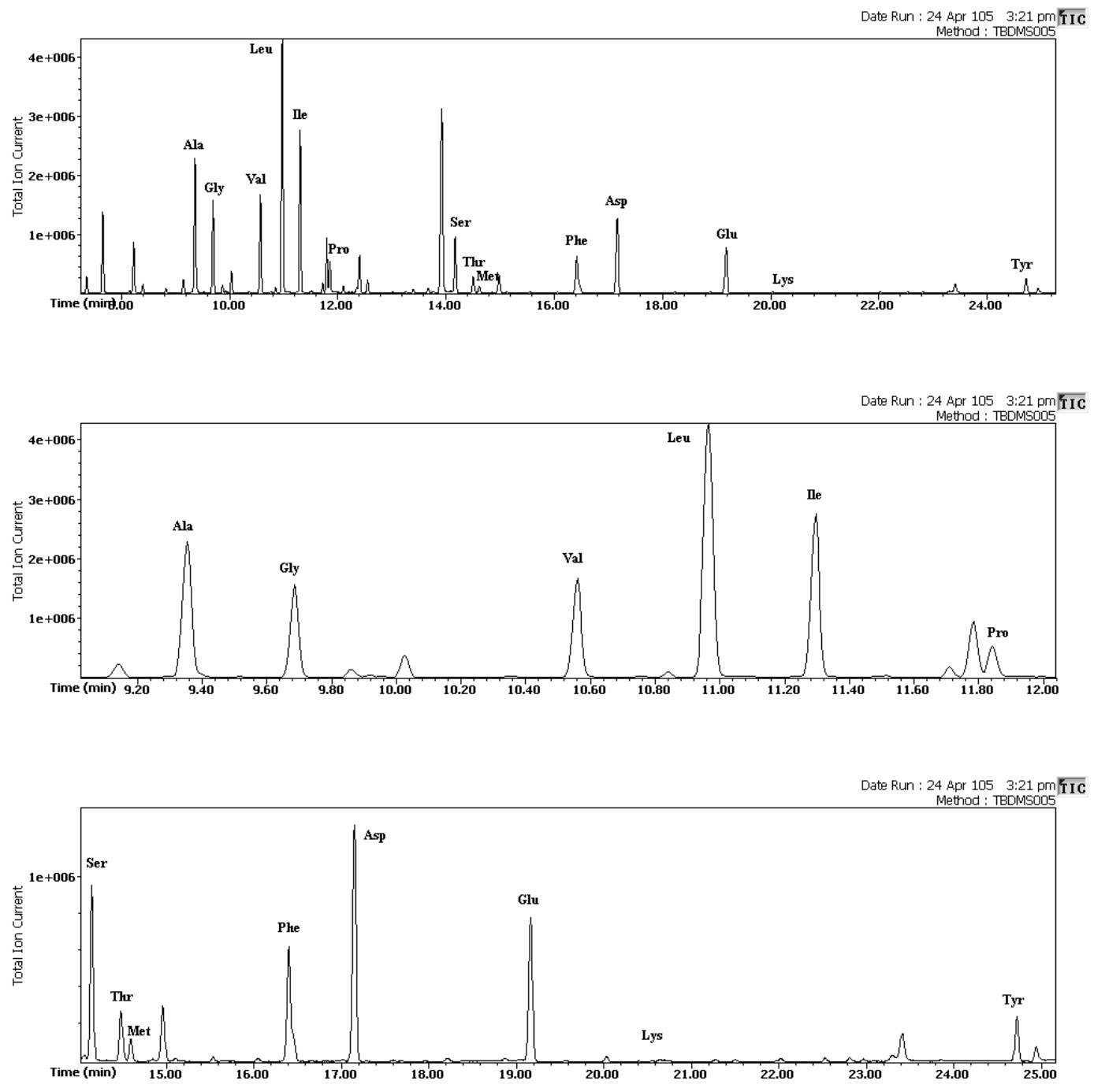


\section{Gas chromatography retention times and main ion fragments of TBDMS-derivatized amino acids}

\begin{tabular}{llll}
\hline Amino acid & Retention time & MW* & Main fragments $(\mathrm{m} / \mathrm{z})$ \\
\hline Alanine & $9.3 \mathrm{~min}$ & 317 & 232,260 \\
Glycine & $9.7 \mathrm{~min}$ & 303 & 218,246 \\
Valine & $10.5 \mathrm{~min}$ & 345 & $260,288,302$ \\
Leucine & $10.9 \mathrm{~min}$ & 359 & $200,274,302$ \\
Isoleucine & $11.3 \mathrm{~min}$ & 359 & $200,274,302$ \\
Proline & $11.8 \mathrm{~min}$ & 343 & 258,286 \\
Serine & $14.1 \mathrm{~min}$ & 447 & $230,288,302,362,390$ \\
Threonine & $14.4 \mathrm{~min}$ & 461 & $376,404,417$ \\
Methionine & $14.6 \mathrm{~min}$ & 377 & $218,244,292,320$ \\
Phenylalanine & $16.4 \mathrm{~min}$ & 393 & $234,302,308,336$ \\
Aspartate & $17.1 \mathrm{~min}$ & 475 & $244,258,302,316,376,390,418$ \\
Glutamate & $19.1 \mathrm{~min}$ & 489 & $272,330,358,404,432$ \\
Lysine & $20.3 \mathrm{~min}$ & 488 & 329,431 \\
Arginine & $22.5 \mathrm{~min}$ & 515 & 340,442 \\
Tyrosine & $24.7 \mathrm{~min}$ & 523 & 302,364 \\
\hline$*$ Molecul & &
\end{tabular}

* Molecular weight of TBDMS-derivatized amino acids. 


\section{Alanine (Ala)}
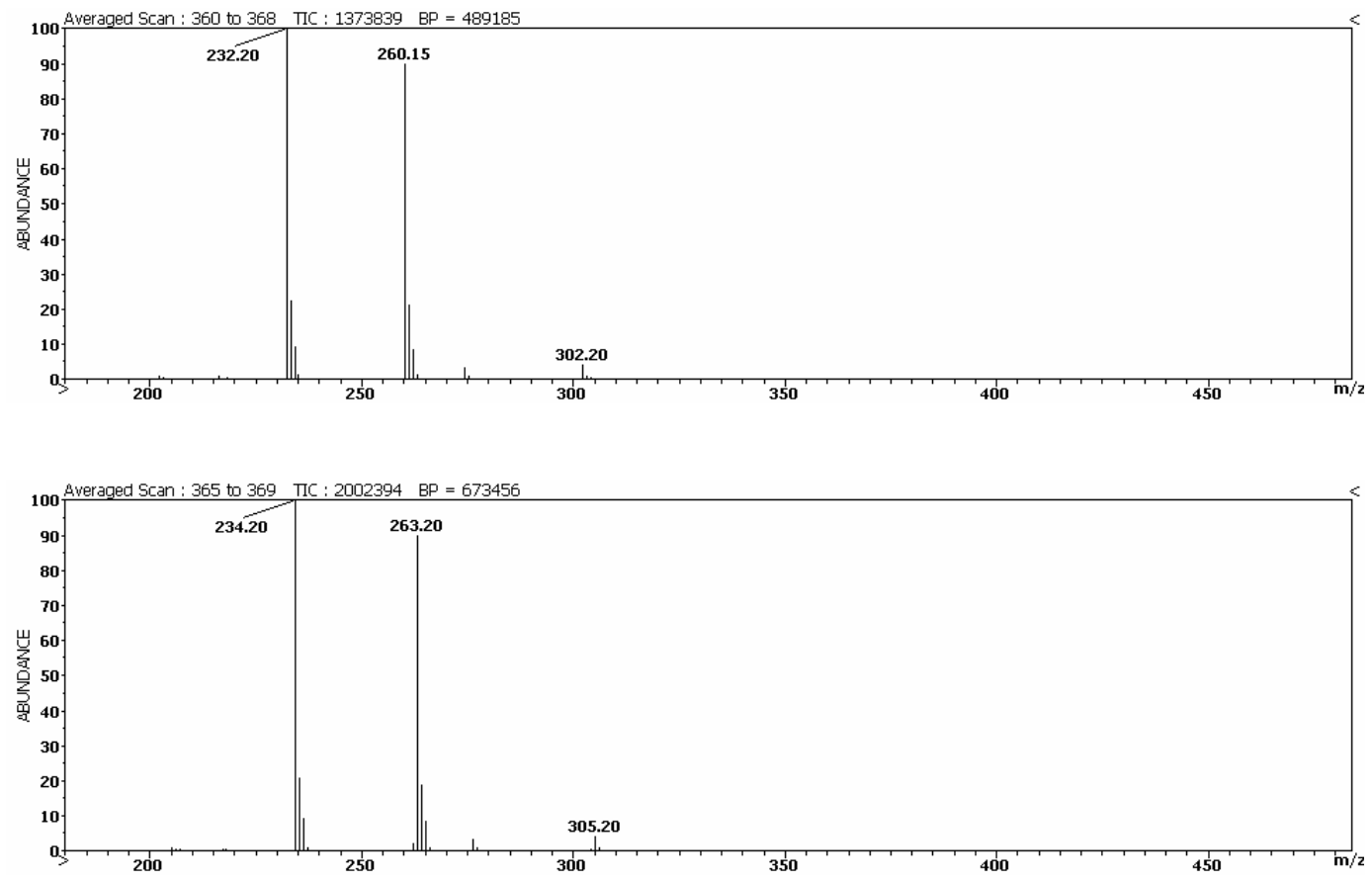

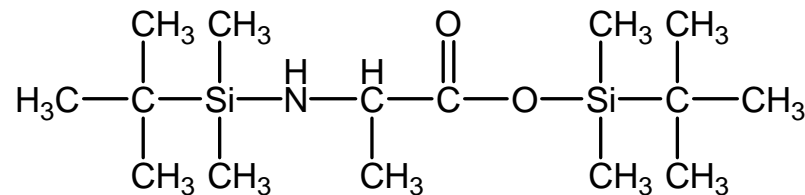

$\mathrm{C}_{15} \mathrm{H}_{35} \mathrm{NO}_{2} \mathrm{Si}_{2}$

Exact Mass: 317.2206 

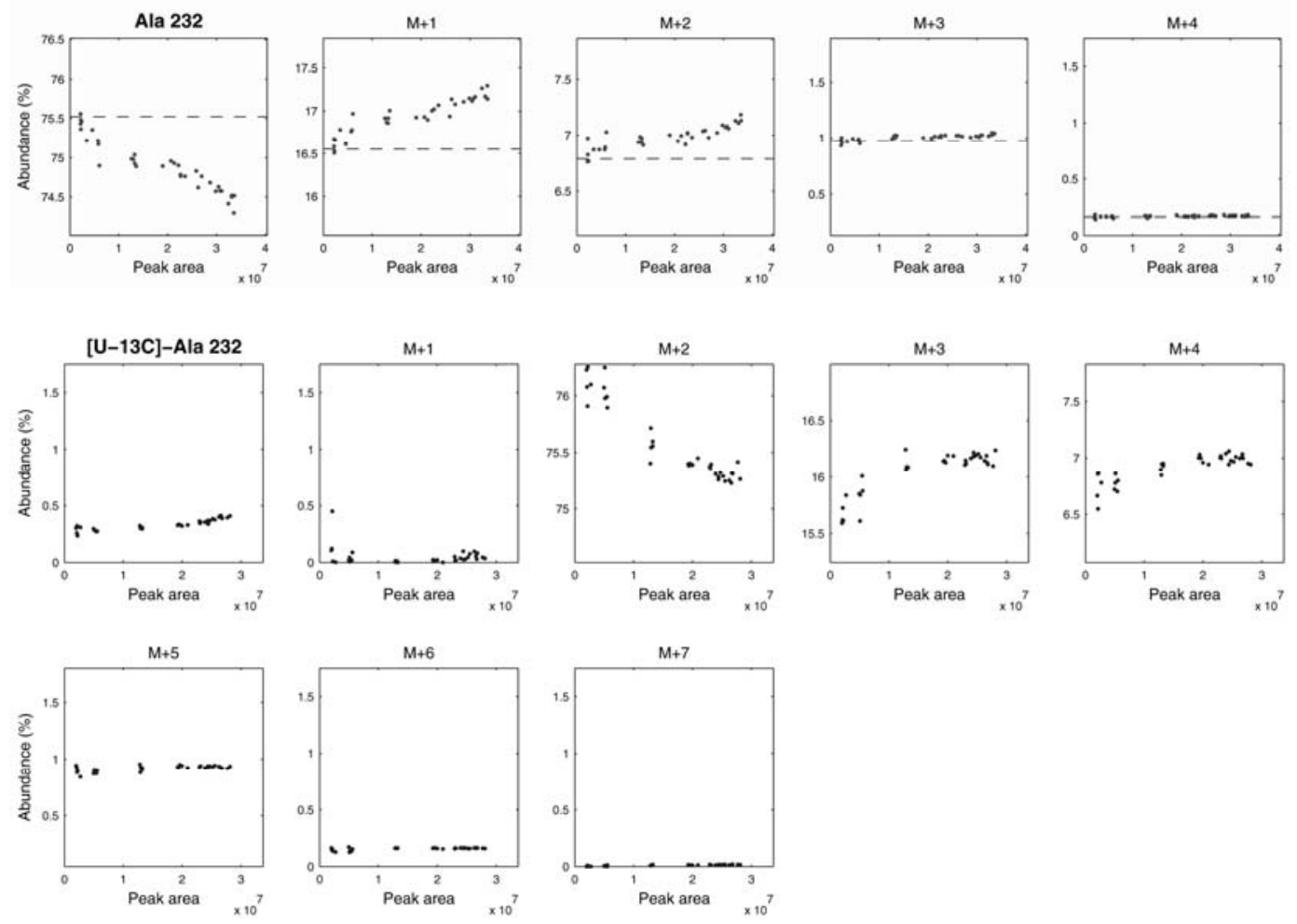

Ala 232

\begin{tabular}{lrrr}
\hline Formula : & \multicolumn{3}{c}{$\mathrm{C}_{10} \mathrm{H}_{26} \mathrm{ONSi}_{2}$} \\
Exact mass : & \multicolumn{3}{c}{232.155} \\
C-atoms : & \multicolumn{3}{c}{$2-3$} \\
\hline $\mathrm{m} / \mathrm{z}$ & theory & data & difference \\
\hline 231 & 0.0 & 0.0 & 0.0 \\
\hline 232 & 100.0 & 100.0 & 0.0 \\
233 & 21.9 & 22.1 & 0.2 \\
234 & 9.0 & 9.1 & 0.1 \\
235 & 1.3 & 1.3 & 0.0 \\
236 & 0.2 & 0.2 & 0.0 \\
237 & 0.0 & 0.0 & 0.0 \\
238 & 0.0 & 0.0 & 0.0 \\
239 & 0.0 & 0.0 & 0.0 \\
\hline
\end{tabular}

$\underline{\left[\mathrm{U}-{ }^{13} \mathrm{C}\right]-\mathrm{Ala}}$

This fragment can be used for flux analysis. 

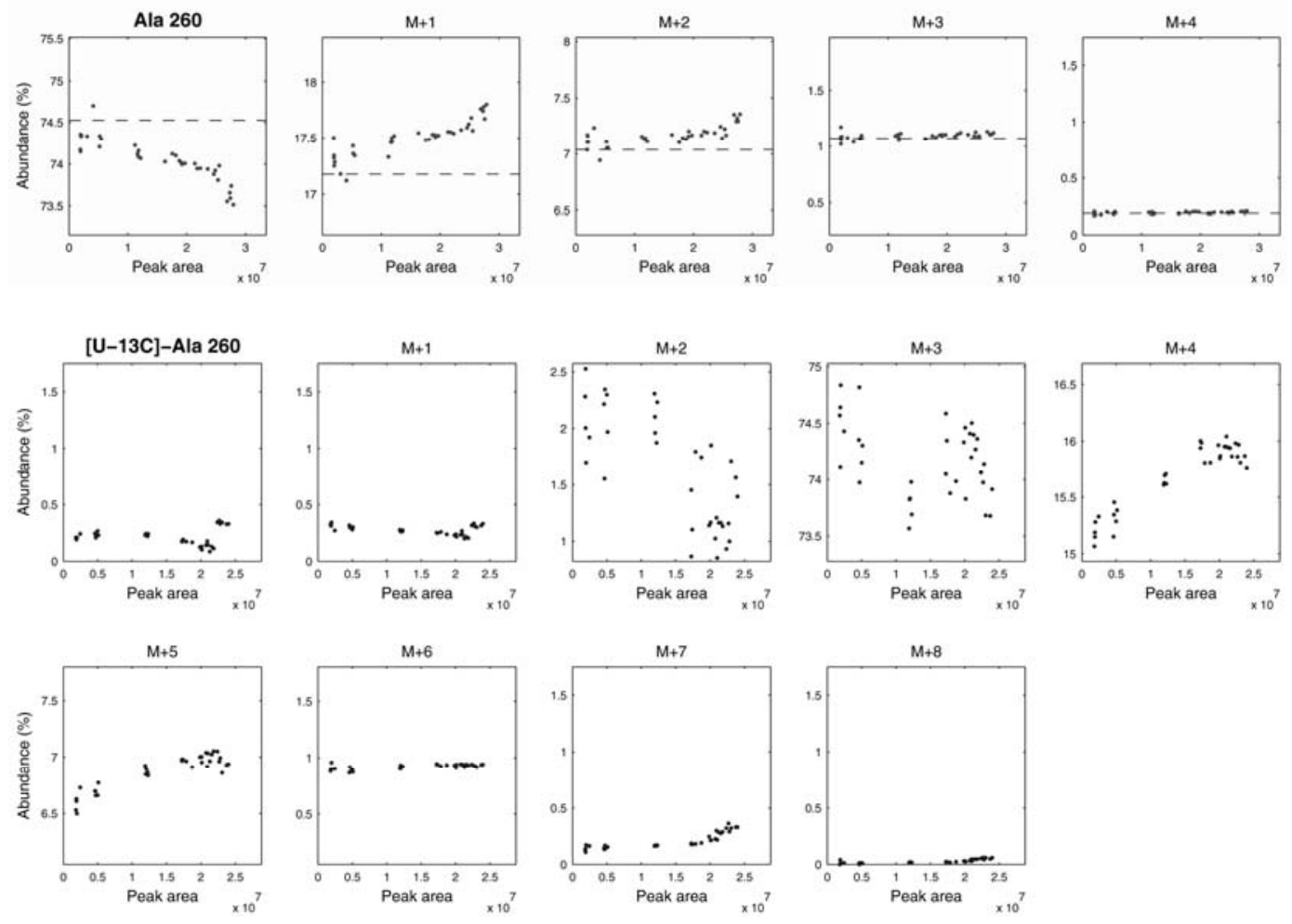

Ala 260

\begin{tabular}{lrrr}
\hline Formula : & \multicolumn{3}{c}{$\mathrm{C}_{11} \mathrm{H}_{26} \mathrm{O}_{2} \mathrm{NSi}_{2}$} \\
Exact mass : & \multicolumn{3}{c}{$1-2$ - 3} \\
C-atoms : & \multicolumn{3}{c}{} \\
\hline $\mathrm{m} / \mathrm{z}$ & theory & data & difference \\
\hline 259 & 0.0 & 0.0 & 0.0 \\
\hline 260 & 100.0 & 100.0 & 0.0 \\
261 & 23.0 & 23.1 & 0.1 \\
262 & 9.4 & 9.4 & 0.0 \\
263 & 1.4 & 1.4 & 0.0 \\
264 & 0.3 & 0.3 & 0.0 \\
265 & 0.0 & 0.0 & 0.0 \\
266 & 0.0 & 0.0 & 0.0 \\
267 & 0.0 & 0.0 & 0.0 \\
268 & 0.0 & 0.0 & 0.0 \\
\hline
\end{tabular}

$\left[\mathrm{U}-{ }^{13} \mathrm{C}\right]-\mathrm{Ala}$

This fragment can be used for flux analysis. 


\section{Glycine (Gly)}
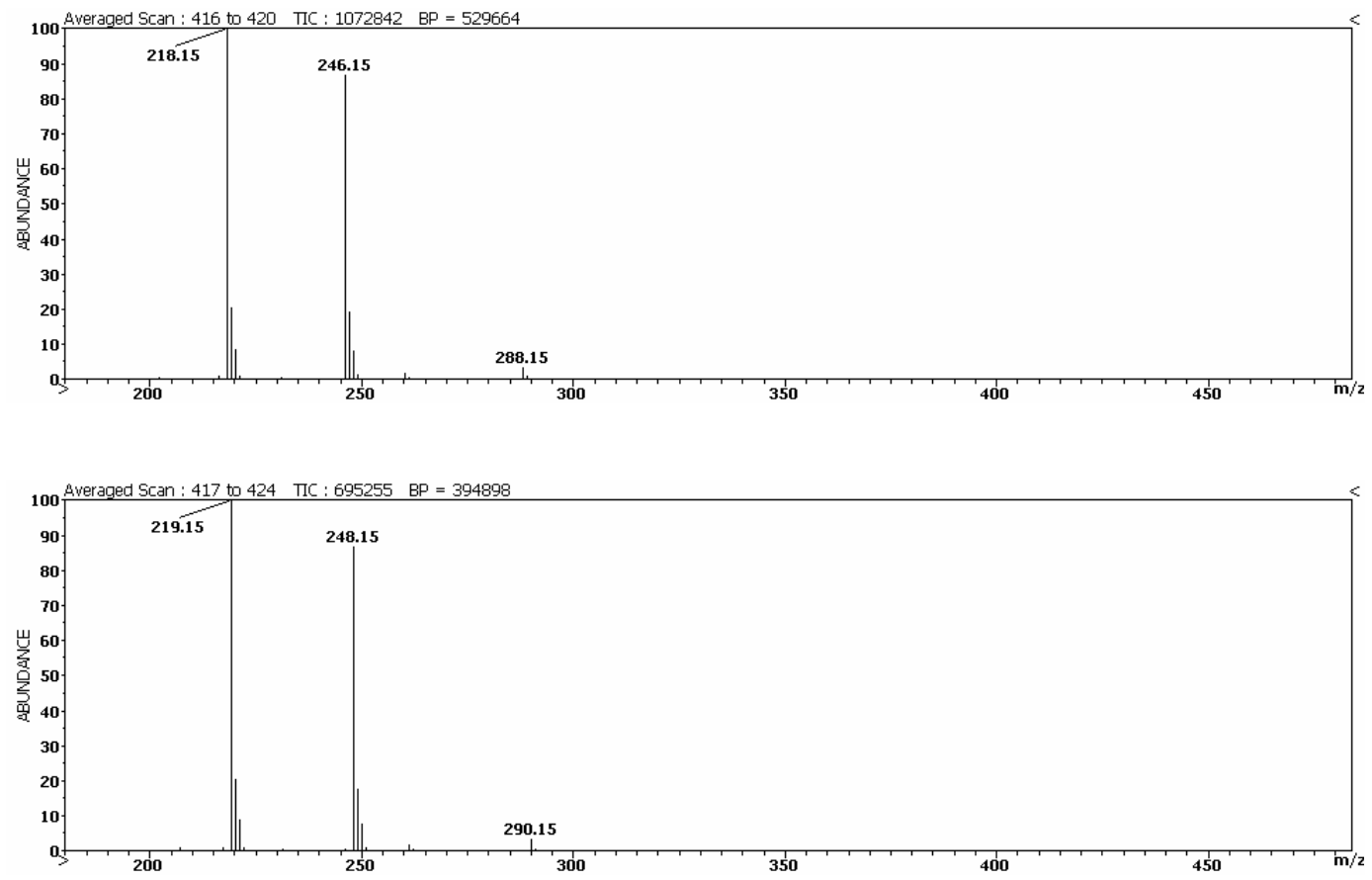

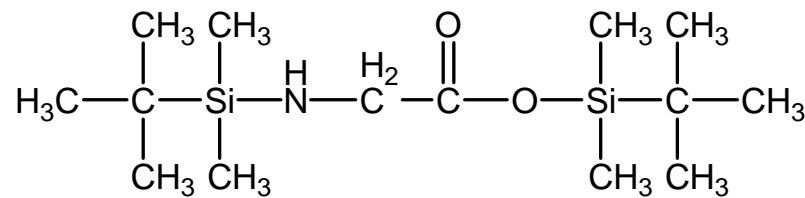

$\mathrm{C}_{14} \mathrm{H}_{33} \mathrm{NO}_{2} \mathrm{Si}_{2}$

Exact Mass: 303.205 

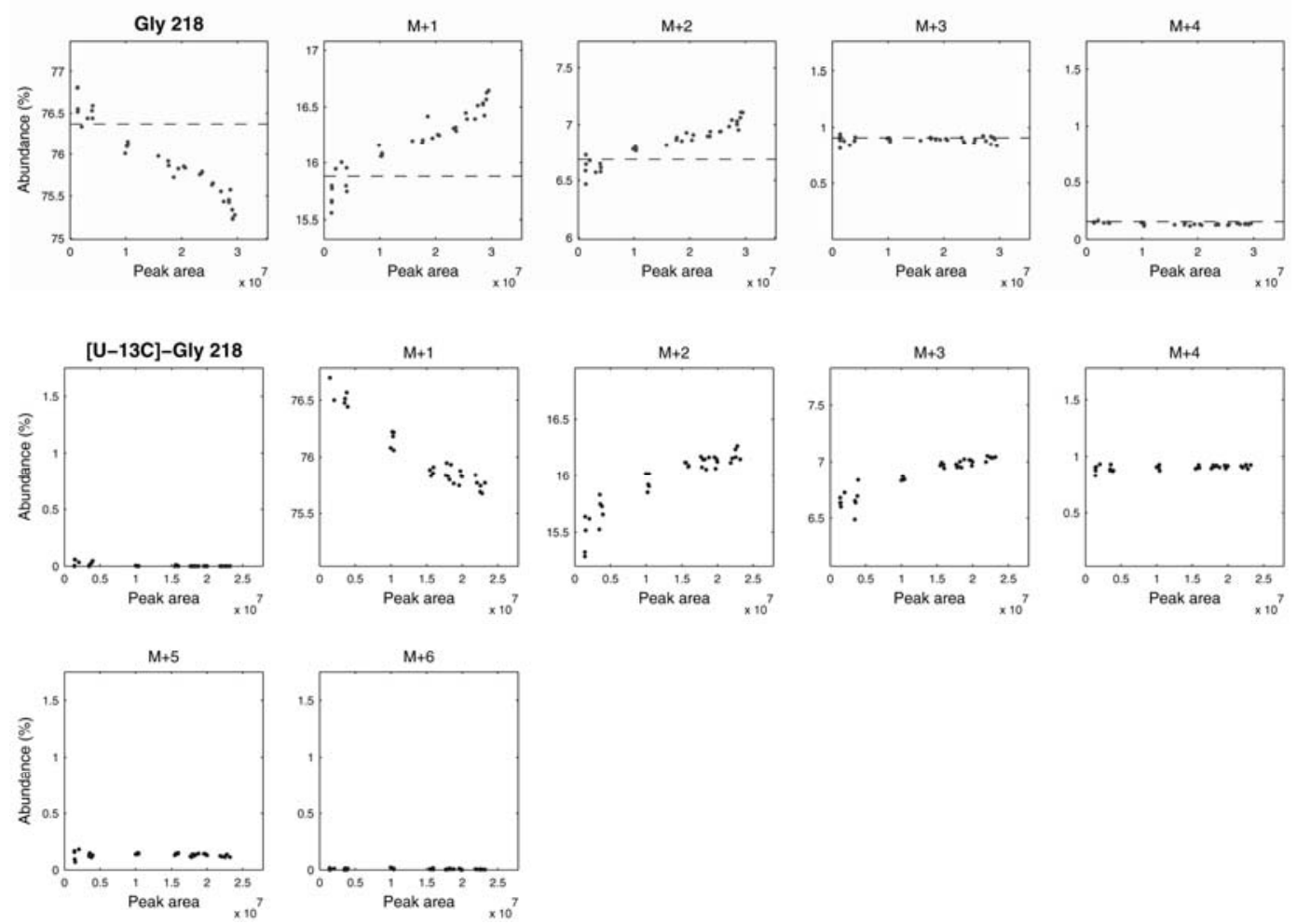

Gly 218

\begin{tabular}{lrrr}
\hline Formula : & \multicolumn{3}{l}{$\mathrm{C}_{9} \mathrm{H}_{24} \mathrm{ONSi}_{2}$} \\
Exact mass : & \multicolumn{3}{l}{218.140} \\
C-atoms : & \multicolumn{2}{c}{2} & \\
\hline $\mathrm{m} / \mathrm{z}$ & theory & data & difference \\
\hline 217 & 0.0 & 0.0 & 0.0 \\
\hline 218 & 100.0 & 100.0 & 0.0 \\
219 & 20.8 & 20.7 & -0.1 \\
220 & 8.7 & 8.6 & -0.1 \\
221 & 1.2 & 1.2 & 0.0 \\
222 & 0.2 & 0.2 & 0.0 \\
223 & 0.0 & 0.0 & 0.0 \\
224 & 0.0 & 0.0 & 0.0 \\
\hline
\end{tabular}

This fragment can be used for flux analysis.

\section{$\left[{ }^{-13} \mathrm{C}\right]-\mathrm{Gly}$}

\begin{tabular}{crrr}
\hline $\mathrm{m} / \mathrm{z}$ & theory & data & difference \\
\hline 217 & 0.0 & 0.9 & 0.9 \\
\hline 218 & 1.0 & 0.0 & -1.0 \\
219 & 100.0 & 100.0 & 0.0 \\
220 & 19.7 & 20.4 & 0.7 \\
221 & 8.5 & 8.7 & 0.2 \\
222 & 1.1 & 1.2 & 0.1 \\
223 & 0.2 & 0.2 & 0.0 \\
224 & 0.0 & 0.0 & 0.0 \\
\hline
\end{tabular}


Gly 246
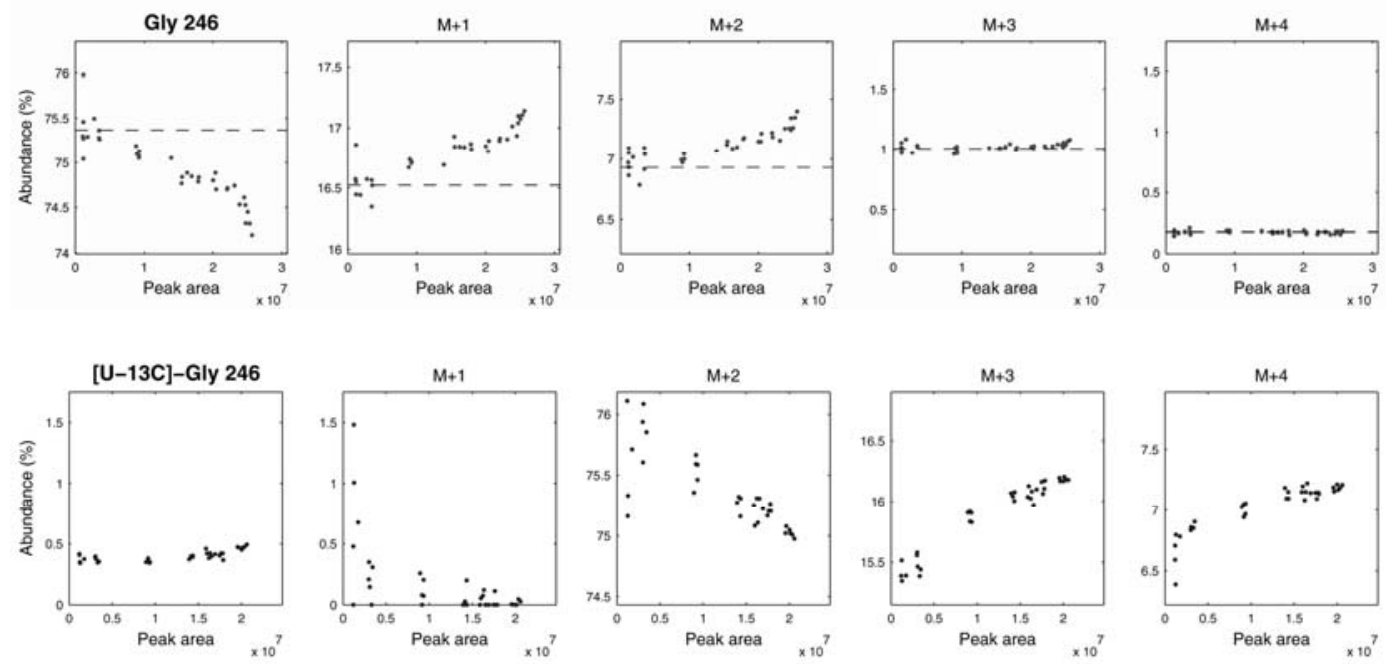
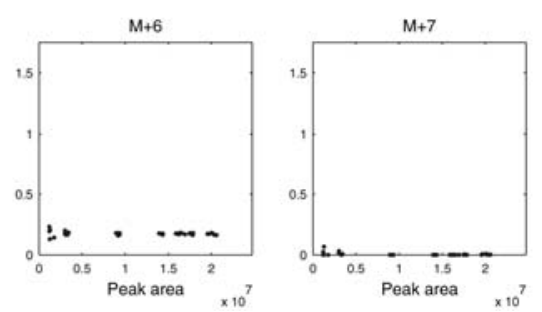

Gly 246

\begin{tabular}{crrr}
\hline Formula : & \multicolumn{3}{c}{$\mathrm{C}_{10} \mathrm{H}_{24} \mathrm{O}_{2} \mathrm{NSi}_{2}$} \\
Exact mass : & \multicolumn{3}{c}{246.135} \\
C-atoms : & \multicolumn{3}{c}{$1-2$} \\
\hline $\mathrm{m} / \mathrm{z}$ & theory & data & difference \\
\hline 245 & 0.0 & 0.0 & 0.0 \\
\hline 246 & 100.0 & 100.0 & 0.0 \\
247 & 21.9 & 21.8 & -0.1 \\
248 & 9.2 & 9.1 & -0.1 \\
249 & 1.3 & 1.3 & 0.0 \\
250 & 0.2 & 0.2 & 0.0 \\
251 & 0.0 & 0.0 & 0.0 \\
252 & 0.0 & 0.0 & 0.0 \\
253 & 0.0 & 0.0 & 0.0 \\
\hline
\end{tabular}

This fragment can be used for flux analysis.
$\left[\mathrm{U}-{ }^{13} \mathrm{C}\right]-\mathrm{Gly}$

\begin{tabular}{crrr}
\hline $\mathrm{m} / \mathrm{z}$ & theory & data & difference \\
\hline 245 & 0.0 & 0.0 & 0.0 \\
\hline 246 & 0.0 & 0.4 & 0.4 \\
247 & 2.0 & 0.6 & -1.4 \\
248 & 100.0 & 100.0 & 0.0 \\
249 & 19.8 & 20.3 & 0.5 \\
250 & 8.7 & 8.9 & 0.2 \\
251 & 1.1 & 1.2 & 0.1 \\
252 & 0.2 & 0.2 & 0.0 \\
253 & 0.0 & 0.0 & 0.0 \\
\hline
\end{tabular}




\section{Valine (Val)}
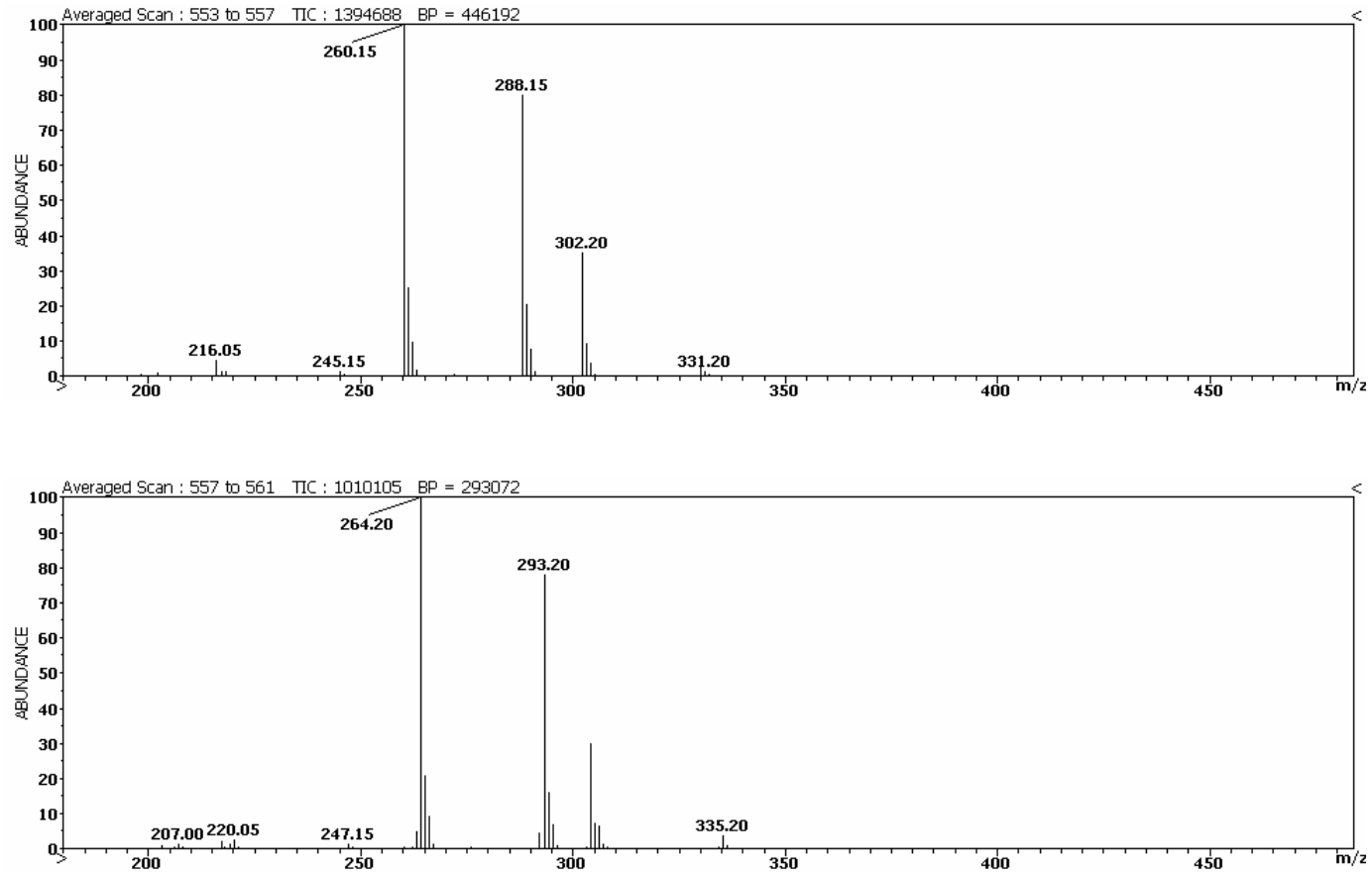<smiles>CC(C)C(N[Si](C)(C)C(C)(C)C)C(=O)O[Si](C)(C)C(C)(C)C</smiles>

$\mathrm{C}_{17} \mathrm{H}_{39} \mathrm{NO}_{2} \mathrm{Si}_{2}$

Exact Mass: 345.2519 

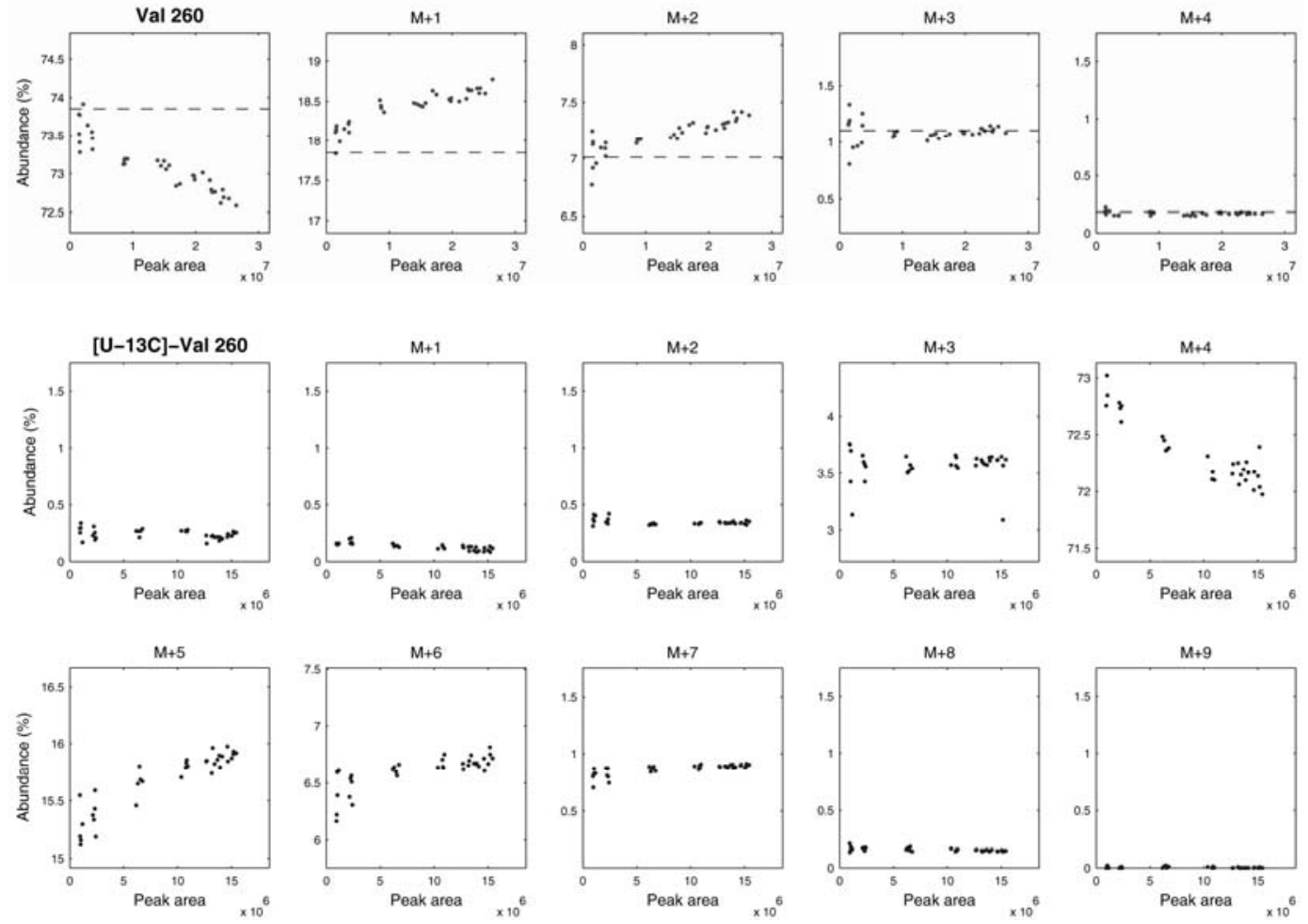

Val 260

\begin{tabular}{lrrr}
\hline Formula: & \multicolumn{3}{c}{$\mathrm{C}_{12} \mathrm{H}_{30} \mathrm{ONSi}_{2}$} \\
Exact mass : & \multicolumn{2}{c}{260.187} \\
C-atoms : & \multicolumn{2}{c}{$2-3-4-5$} & \\
\hline $\mathrm{m} / \mathrm{z}$ & theory & data & difference \\
\hline 259 & 0.0 & 0.0 & 0.0 \\
\hline 260 & 100.0 & 100.0 & 0.0 \\
261 & 24.2 & 24.7 & 0.5 \\
262 & 9.5 & 9.6 & 0.1 \\
263 & 1.5 & 1.6 & 0.1 \\
264 & 0.2 & 0.2 & 0.0 \\
265 & 0.0 & 0.0 & 0.0 \\
266 & 0.0 & 0.0 & 0.0 \\
267 & 0.0 & 0.0 & 0.0 \\
268 & 0.0 & 0.0 & 0.0 \\
269 & 0.0 & 0.0 & 0.0 \\
\hline
\end{tabular}

\section{$\left[\mathrm{U}-{ }^{13} \mathrm{C}\right]-\mathrm{Val}$}

\begin{tabular}{rrrr}
\hline $\mathrm{m} / \mathrm{z}$ & theory & data & difference \\
\hline 259 & 0.0 & 0.0 & 0.0 \\
\hline 260 & 0.0 & 0.4 & 0.4 \\
261 & 0.0 & 0.2 & 0.2 \\
262 & 0.0 & 0.5 & 0.5 \\
263 & 4.1 & 4.9 & 0.8 \\
264 & 100.0 & 100.0 & 0.0 \\
265 & 20.0 & 21.1 & 1.1 \\
266 & 8.5 & 8.9 & 0.4 \\
267 & 1.1 & 1.1 & 0.0 \\
268 & 0.2 & 0.2 & 0.0 \\
269 & 0.0 & 0.0 & 0.0 \\
\hline
\end{tabular}

This fragment can be used for flux analysis. 

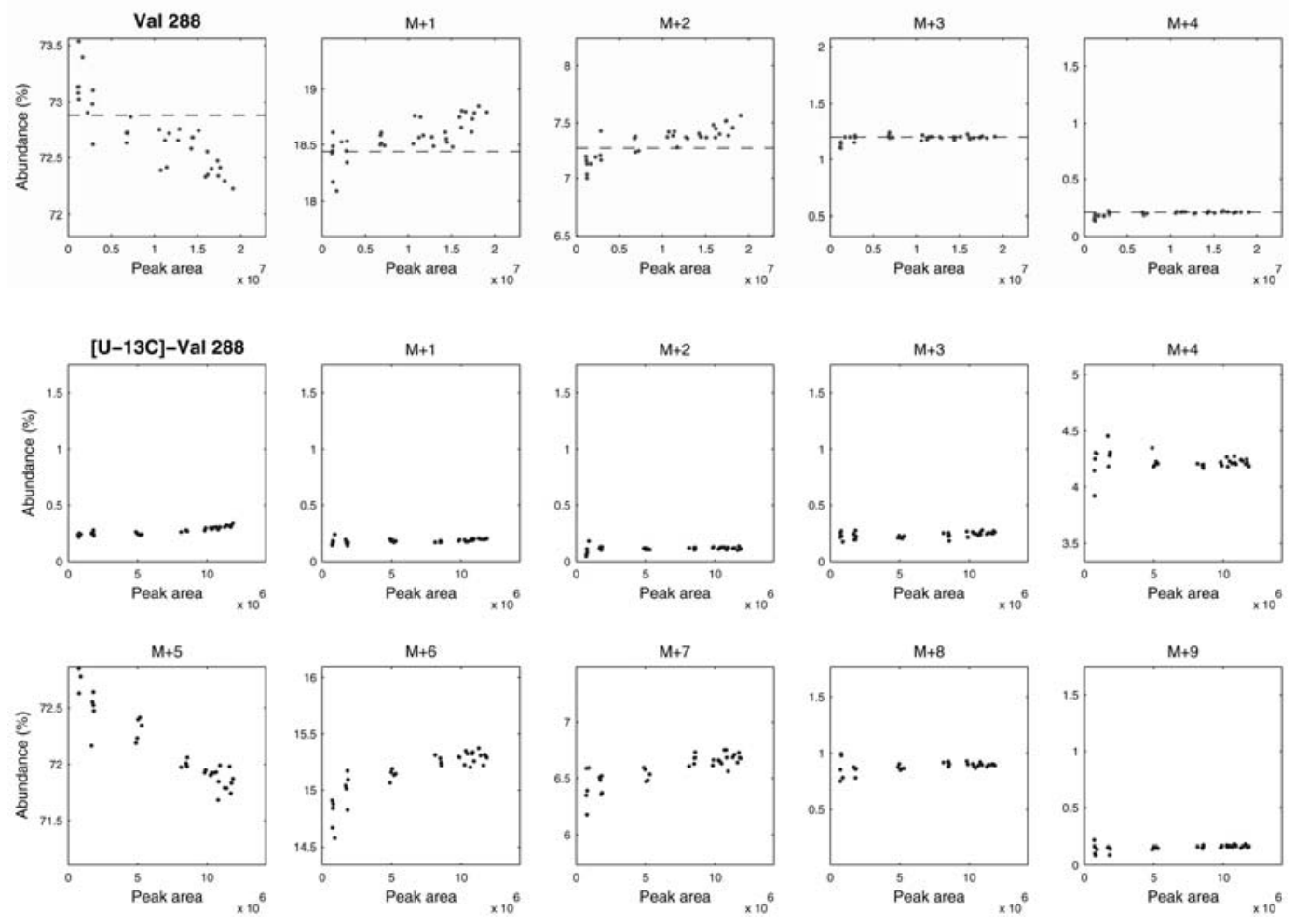

Val 288

\begin{tabular}{lrrr}
\hline Formula : & \multicolumn{3}{c}{$\mathrm{C}_{13} \mathrm{H}_{30} \mathrm{O}_{2} \mathrm{NSi}_{2}$} \\
Exact mass : & \multicolumn{3}{c}{288.182} \\
C-atoms : & \multicolumn{3}{c}{$1-2-3-4-5$} \\
\hline $\mathrm{m} / \mathrm{z}$ & theory & data & difference \\
\hline 287 & 0.0 & 0.0 & 0.0 \\
\hline 288 & 100.0 & 100.0 & 0.0 \\
289 & 25.3 & 25.2 & -0.1 \\
290 & 10.0 & 9.8 & -0.2 \\
291 & 1.6 & 1.6 & 0.0 \\
292 & 0.3 & 0.2 & -0.1 \\
293 & 0.0 & 0.0 & 0.0 \\
294 & 0.0 & 0.0 & 0.0 \\
295 & 0.0 & 0.0 & 0.0 \\
296 & 0.0 & 0.0 & 0.0 \\
297 & 0.0 & 0.0 & 0.0 \\
298 & 0.0 & 0.0 & 0.0 \\
\hline
\end{tabular}

\section{$\left[\mathrm{U}-{ }^{13} \mathrm{C}\right]-\mathrm{Val}$}

\begin{tabular}{crrr}
\hline $\mathrm{m} / \mathrm{z}$ & theory & data & difference \\
\hline 287 & 0.0 & 0.0 & 0.0 \\
\hline 288 & 0.0 & 0.3 & 0.3 \\
289 & 0.0 & 0.2 & 0.2 \\
290 & 0.0 & 0.1 & 0.1 \\
291 & 0.0 & 0.3 & 0.3 \\
292 & 5.2 & 5.8 & 0.6 \\
293 & 100.0 & 100.0 & 0.0 \\
294 & 20.1 & 20.5 & 0.4 \\
295 & 8.7 & 8.8 & 0.1 \\
296 & 1.1 & 1.2 & 0.1 \\
297 & 0.2 & 0.2 & 0.0 \\
298 & 0.0 & 0.0 & 0.0 \\
\hline
\end{tabular}

This fragment can be used for flux analysis. 

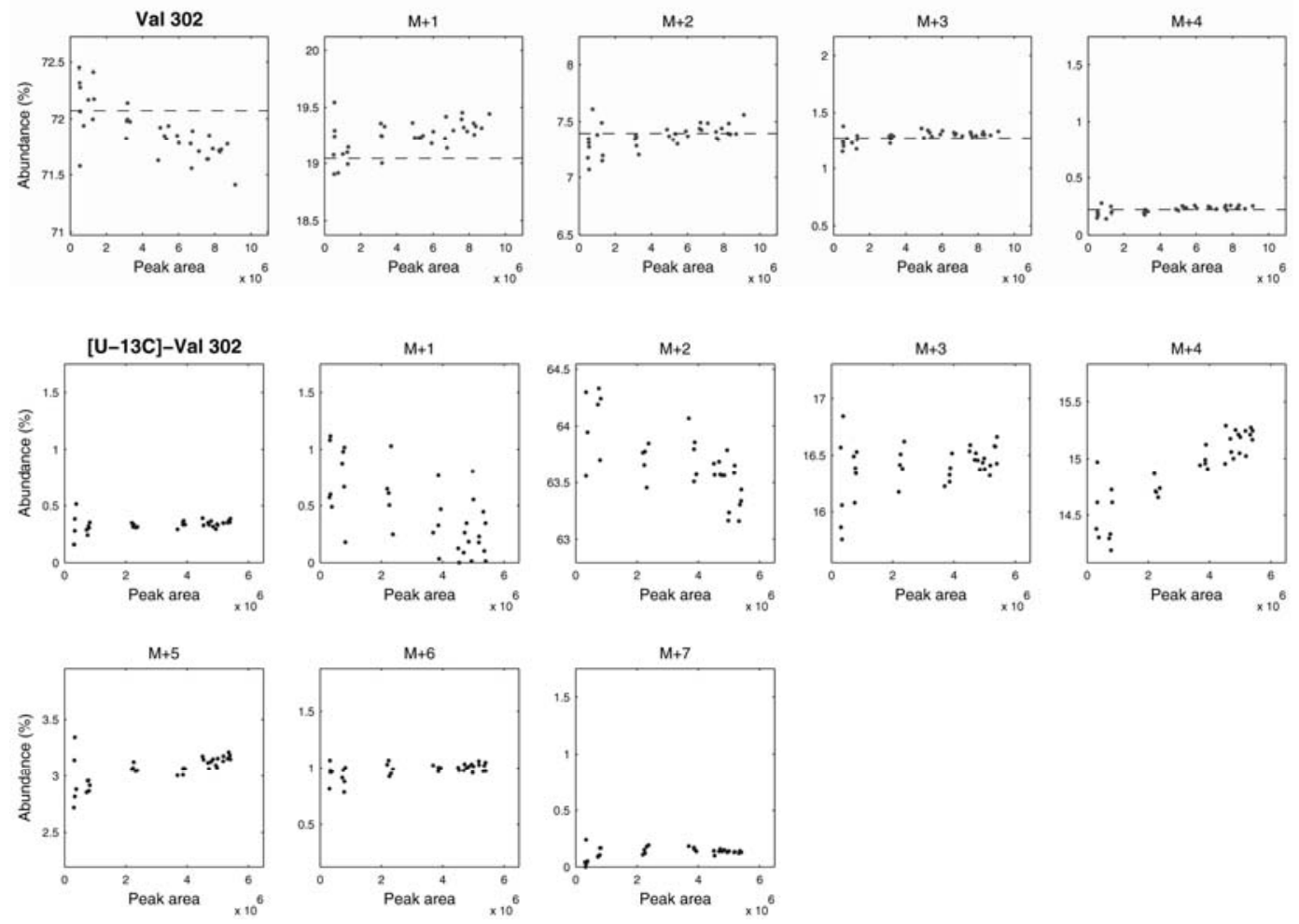

Val 302

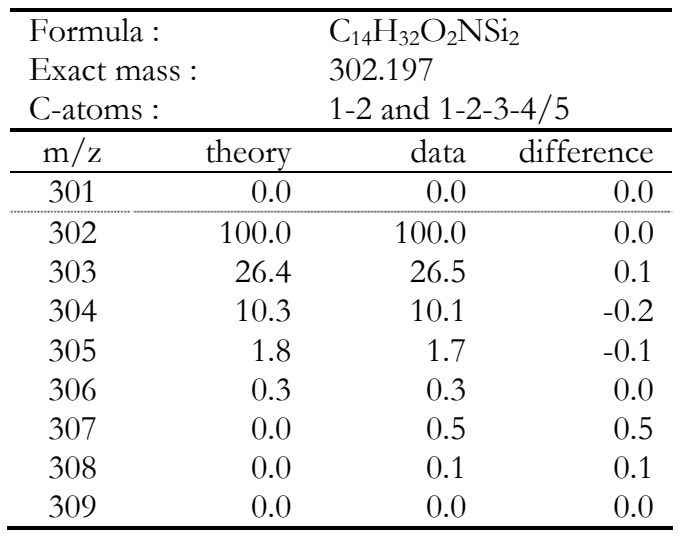

$\left[\mathrm{U}-{ }^{13} \mathrm{C}\right]-\mathrm{Val}$

This fragment should not be used for flux analysis. The presence of $\mathrm{M}+2$ and $\mathrm{M}+4$ abundances in [U-

$\left.{ }^{13} \mathrm{C}\right]$ valine suggests that two different fragments are overlapping. 


\section{Leucine (Leu)}
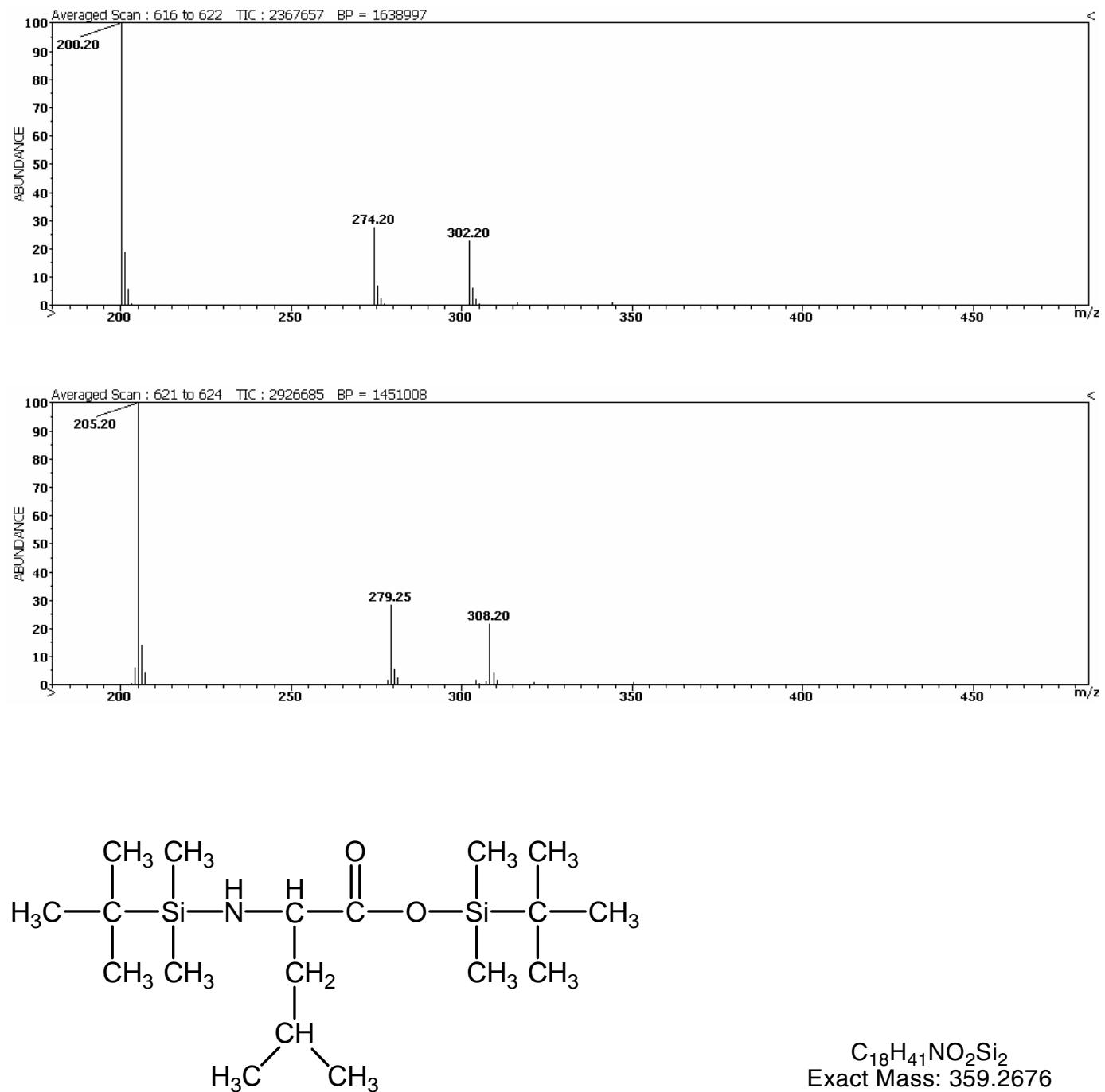

$\mathrm{C}_{18} \mathrm{H}_{41} \mathrm{NO}_{2} \mathrm{Si}_{2}$

Exact Mass: 359.2676 

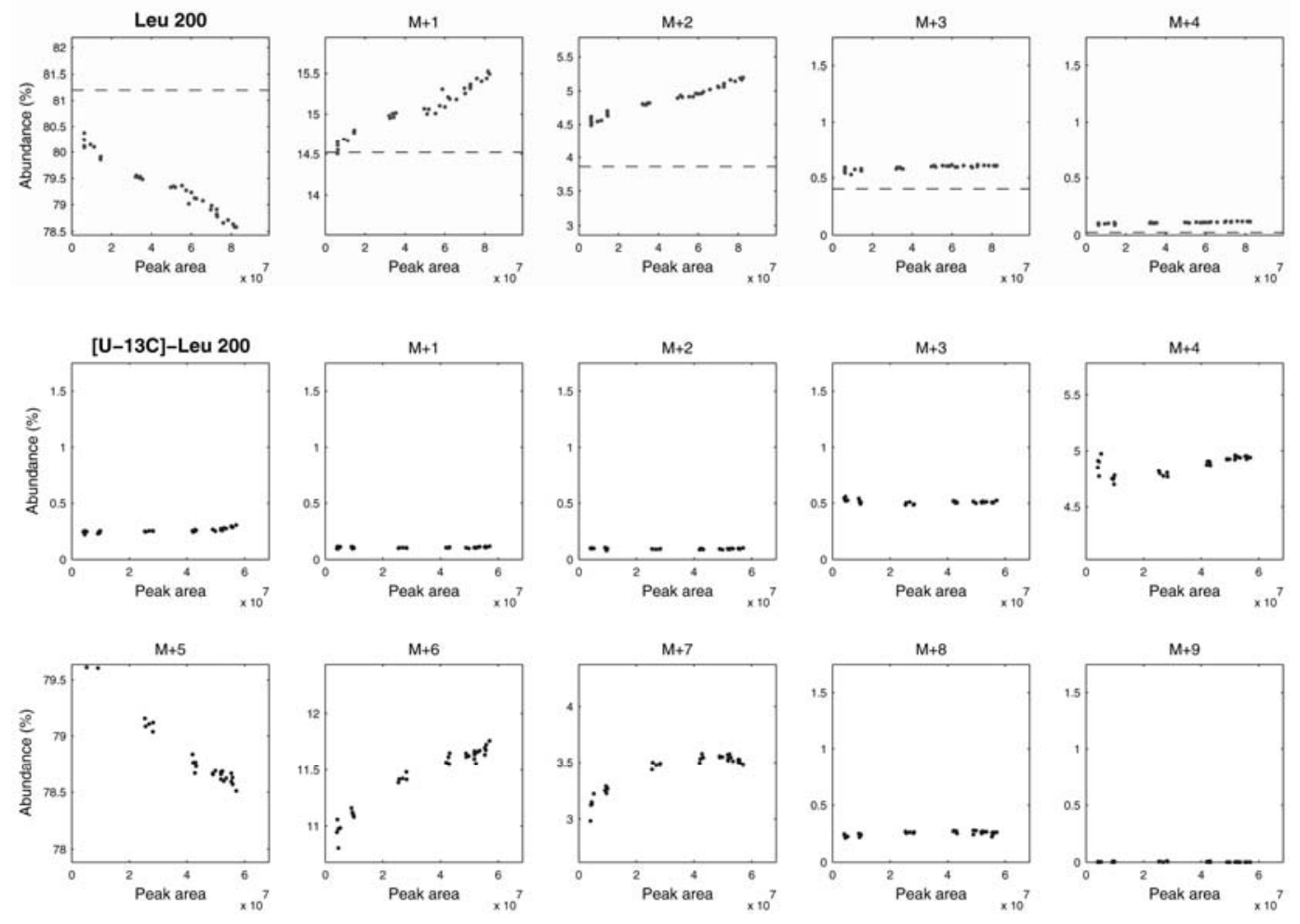

Leu 200

\begin{tabular}{lrrr}
\hline Formula : & \multicolumn{2}{l}{$\mathrm{C}_{11} \mathrm{H}_{26} \mathrm{NSi}$} \\
$\begin{array}{l}\text { Exact mass : } \\
\text { C-atoms : }\end{array}$ & & 200.183 & \\
\hline $\mathrm{m} / \mathrm{z}$ & theory & data & difference \\
\hline 199 & 0.0 & 0.0 & 0.0 \\
\hline 200 & 100.0 & 100.0 & 0.0 \\
201 & 17.9 & 18.2 & 0.3 \\
202 & 4.8 & 5.6 & 0.8 \\
203 & 0.5 & 0.7 & 0.2 \\
204 & 0.0 & 0.1 & 0.1 \\
205 & 0.0 & 0.0 & 0.0 \\
206 & 0.0 & 0.0 & 0.0 \\
207 & 0.0 & 0.0 & 0.0 \\
208 & 0.0 & 0.0 & 0.0 \\
209 & 0.0 & 0.0 & 0.0 \\
\hline
\end{tabular}

\section{$\left[{ }^{U-13} \mathrm{C}\right]-$ Leu}

\begin{tabular}{rrrr}
\hline $\mathrm{m} / \mathrm{z}$ & theory & data & difference \\
\hline 199 & 0.0 & 0.0 & 0.0 \\
\hline 200 & 0.0 & 0.3 & 0.3 \\
201 & 0.0 & 0.1 & 0.1 \\
202 & 0.0 & 0.1 & 0.1 \\
203 & 0.0 & 0.6 & 0.6 \\
204 & 5.2 & 5.9 & 0.7 \\
205 & 100.0 & 100.0 & 0.0 \\
206 & 12.5 & 13.8 & 1.3 \\
207 & 3.9 & 4.1 & 0.2 \\
208 & 0.3 & 0.3 & 0.0 \\
209 & 0.0 & 0.0 & 0.0 \\
\hline
\end{tabular}

This fragment should not be used for flux analysis. This fragment has a significant bias, i.e. $\mathrm{M}+0$ is too low $(-1.0 \mathrm{~mol} \%)$ and $\mathrm{M}+2$ is too high $(+0.7 \mathrm{~mol} \%)$. Fragment Leu-274 is preferred. 

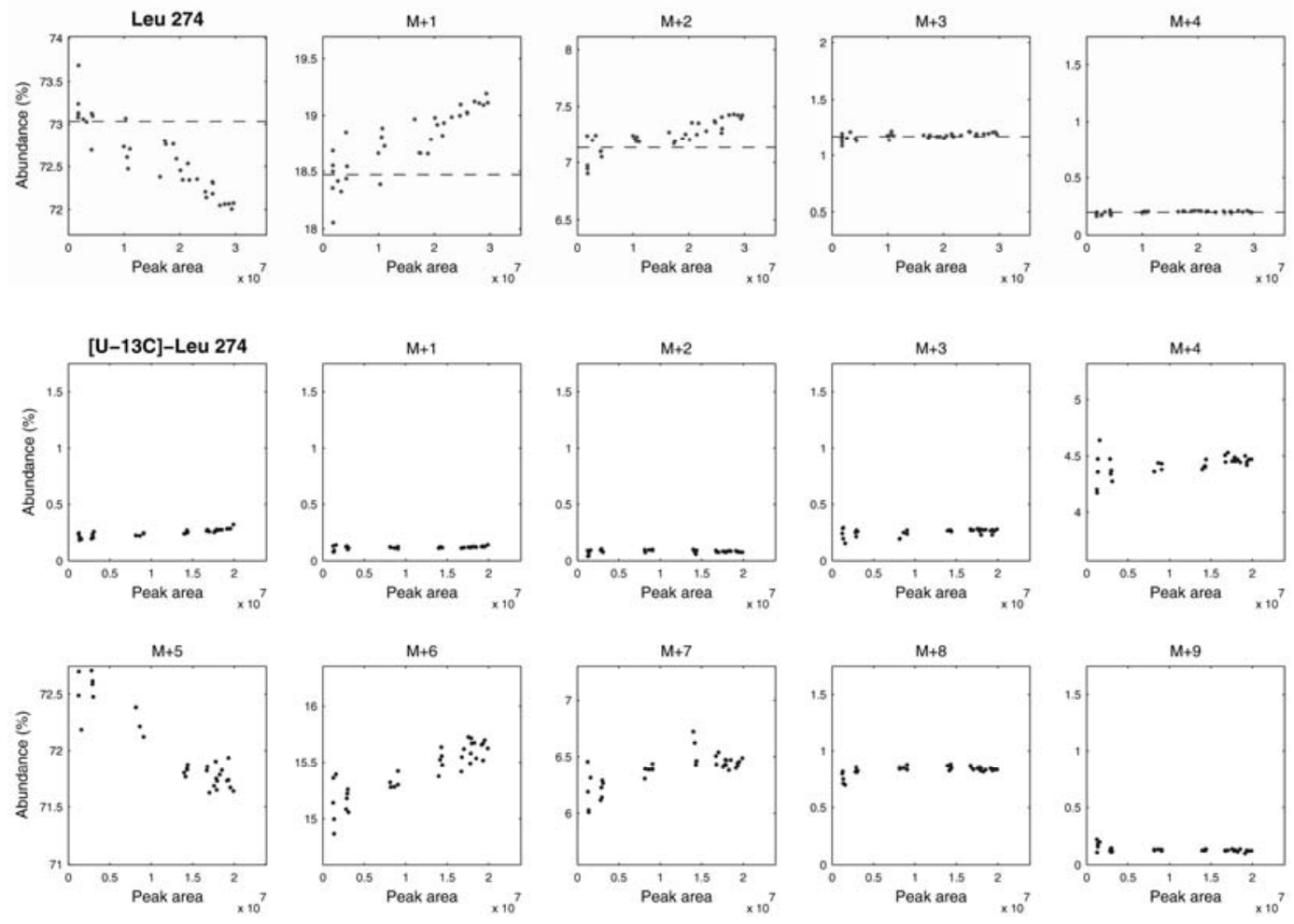

Leu 274

\begin{tabular}{lrrr}
\hline Formula : & \multicolumn{3}{c}{$\mathrm{C}_{13} \mathrm{H}_{32} \mathrm{ONSi}_{2}$} \\
Exact mass : & \multicolumn{3}{c}{274.202} \\
C-atoms : & \multicolumn{2}{c}{$2-3-4-5-6$} \\
m/z & theory & data & difference \\
\hline 273 & 0.0 & 0.0 & 0.0 \\
\hline 274 & 100.0 & 100.0 & 0.0 \\
275 & 25.3 & 25.1 & -0.2 \\
276 & 9.8 & 9.6 & -0.2 \\
277 & 1.6 & 1.6 & 0.0 \\
278 & 0.3 & 0.3 & 0.0 \\
279 & 0.0 & 0.0 & 0.0 \\
280 & 0.0 & 0.0 & 0.0 \\
281 & 0.0 & 0.0 & 0.0 \\
282 & 0.0 & 0.0 & 0.0 \\
283 & 0.0 & 0.0 & 0.0 \\
284 & 0.0 & 0.1 & 0.1 \\
\hline
\end{tabular}

$\left[\mathrm{U}-{ }^{13} \mathrm{C}\right]-$ Leu

This fragment can be used for flux analysis. 

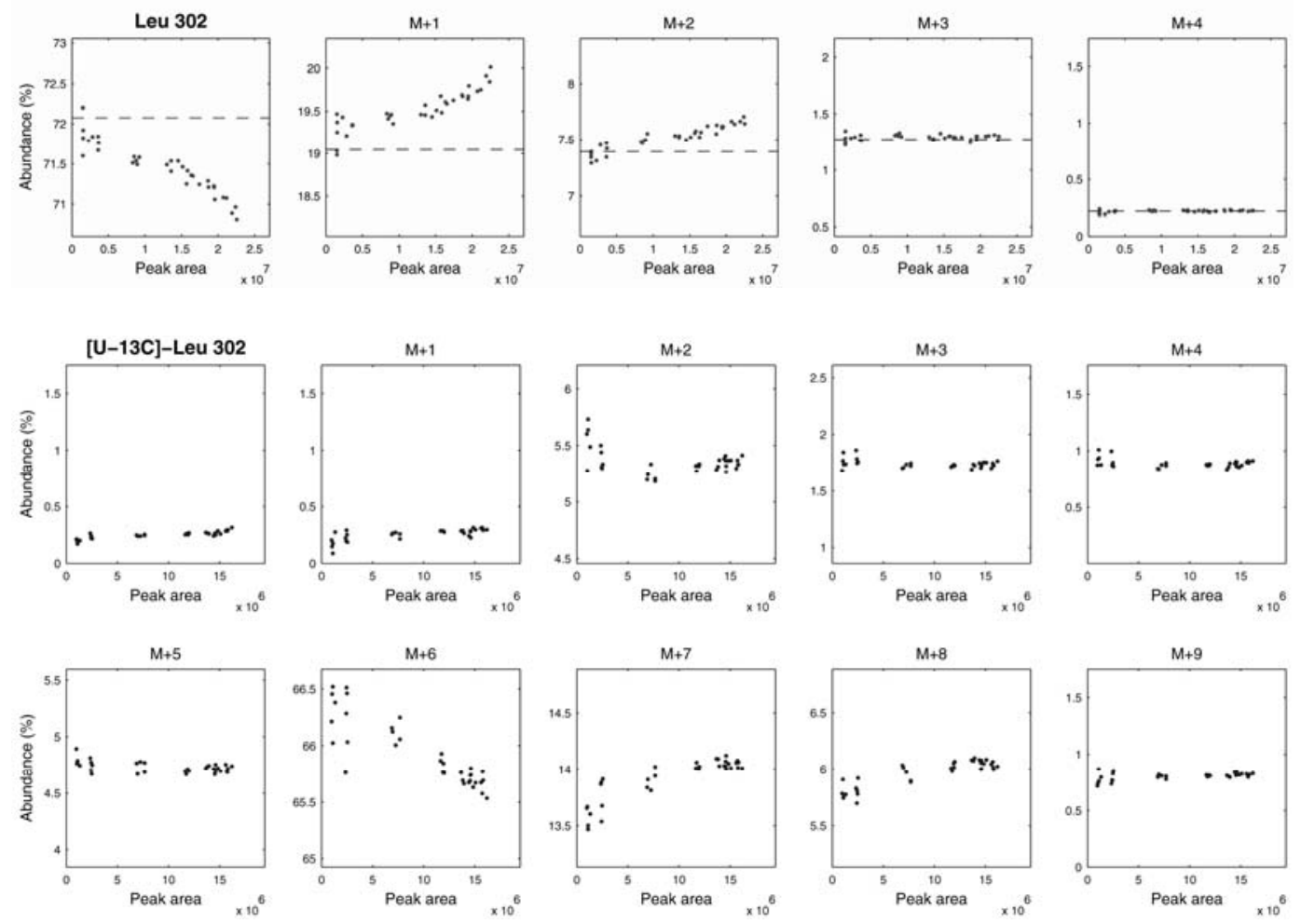

Leu 302

\begin{tabular}{|c|c|c|c|}
\hline \multicolumn{2}{|c|}{$\begin{array}{l}\text { Formula : } \\
\text { Exact mass : } \\
\text { C-atoms : }\end{array}$} & \multicolumn{2}{|c|}{$\begin{array}{l}\mathrm{C}_{14} \mathrm{H}_{32} \mathrm{O}_{2} \mathrm{NSi}_{2} \\
302.197 \\
1-2 \text { and 1-2-3-4-5-6 }\end{array}$} \\
\hline $\mathrm{m} / \mathrm{z}$ & theory & data & difference \\
\hline 301 & 0.0 & 0.0 & 0.0 \\
\hline 302 & 100.0 & 100.0 & 0.0 \\
\hline 303 & 26.4 & 26.6 & 0.2 \\
\hline 304 & 10.3 & 10.2 & -0.1 \\
\hline 305 & 1.8 & 1.8 & 0.0 \\
\hline 306 & 0.3 & 0.3 & 0.0 \\
\hline 307 & 0.0 & 0.0 & 0.0 \\
\hline 308 & 0.0 & 0.0 & 0.0 \\
\hline 309 & 0.0 & 0.0 & 0.0 \\
\hline 310 & 0.0 & 0.0 & 0.0 \\
\hline 311 & 0.0 & 0.0 & 0.0 \\
\hline 312 & 0.0 & 0.0 & 0.0 \\
\hline 313 & 0.0 & 0.0 & 0.0 \\
\hline
\end{tabular}

$\left[\mathrm{U}-{ }^{13} \mathrm{C}\right]-$ Leu

This fragment should not be used for flux analysis. The presence of $\mathrm{M}+2$ and $\mathrm{M}+6$ abundances in [U$\left.{ }^{13} \mathrm{C}\right]$ leucine suggests that two different fragments are overlapping. 


\section{Isoleucine (Ile)}
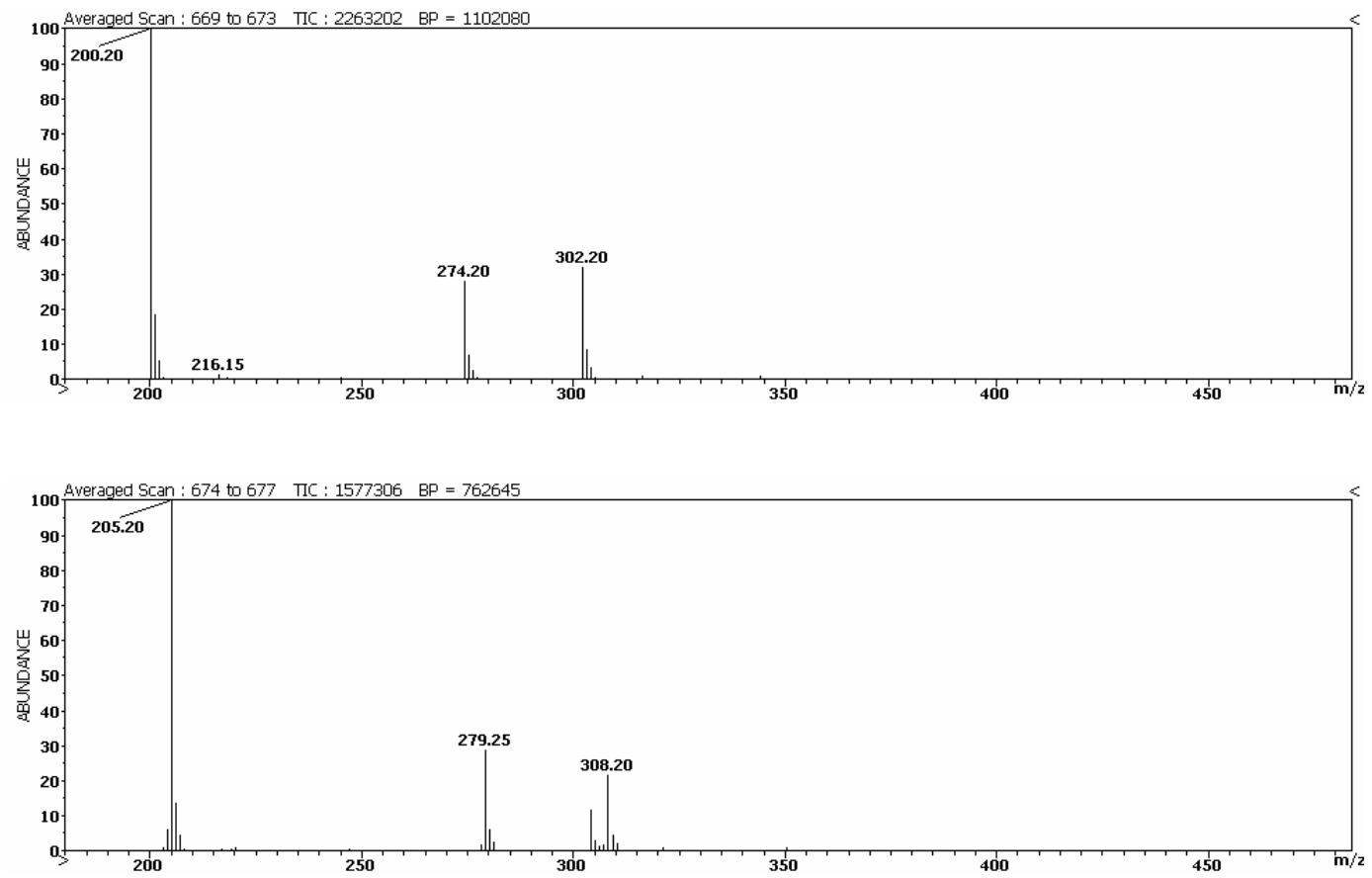<smiles>CCC(C)C(N[Si](C)(C)C(C)(C)C)C(=O)O[Si](C)(C)C(C)(C)C</smiles>

$\mathrm{C}_{18} \mathrm{H}_{41} \mathrm{NO}_{2} \mathrm{Si}_{2}$

Exact Mass: 359.2676 

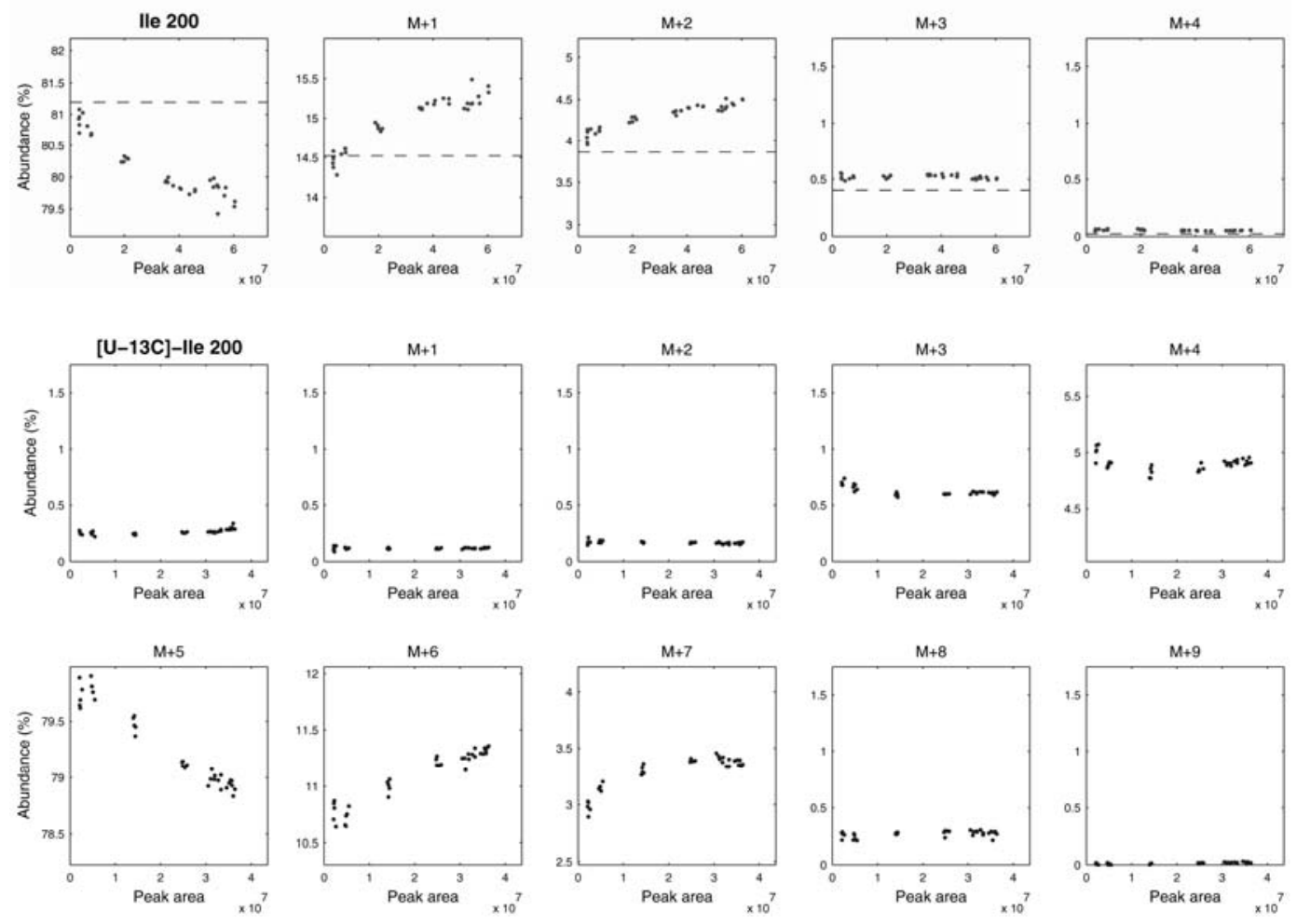

Ile 200

\begin{tabular}{lrrr}
\hline Formula : & \multicolumn{2}{l}{$\mathrm{C}_{11} \mathrm{H}_{26} \mathrm{NSi}$} \\
Exact mass : & & 200.183 \\
C-atoms : & & $2-3-4-5-6$ & \\
\hline $\mathrm{m} / \mathrm{z}$ & theory & data & difference \\
\hline 199 & 0.0 & 0.0 & 0.0 \\
\hline 200 & 100.0 & 100.0 & 0.0 \\
201 & 17.9 & 18.0 & 0.1 \\
202 & 4.8 & 5.1 & 0.3 \\
203 & 0.5 & 0.6 & 0.1 \\
204 & 0.0 & 0.1 & 0.1 \\
205 & 0.0 & 0.0 & 0.0 \\
206 & 0.0 & 0.0 & 0.0 \\
207 & 0.0 & 0.0 & 0.0 \\
208 & 0.0 & 0.0 & 0.0 \\
209 & 0.0 & 0.0 & 0.0 \\
\hline
\end{tabular}

[U-13C]-Ile

\begin{tabular}{crrr}
\hline $\mathrm{m} / \mathrm{z}$ & theory & data & difference \\
\hline 199 & 0.0 & 0.0 & 0.0 \\
\hline 200 & 0.0 & 0.3 & 0.3 \\
201 & 0.0 & 0.1 & 0.1 \\
202 & 0.0 & 0.2 & 0.2 \\
203 & 0.0 & 0.8 & 0.8 \\
204 & 5.2 & 6.1 & 0.9 \\
205 & 100.0 & 100.0 & 0.0 \\
206 & 12.5 & 13.4 & 0.9 \\
207 & 3.9 & 3.9 & 0.0 \\
208 & 0.3 & 0.3 & 0.0 \\
209 & 0.0 & 0.0 & 0.0 \\
\hline
\end{tabular}

This fragment can be used for flux analysis. 

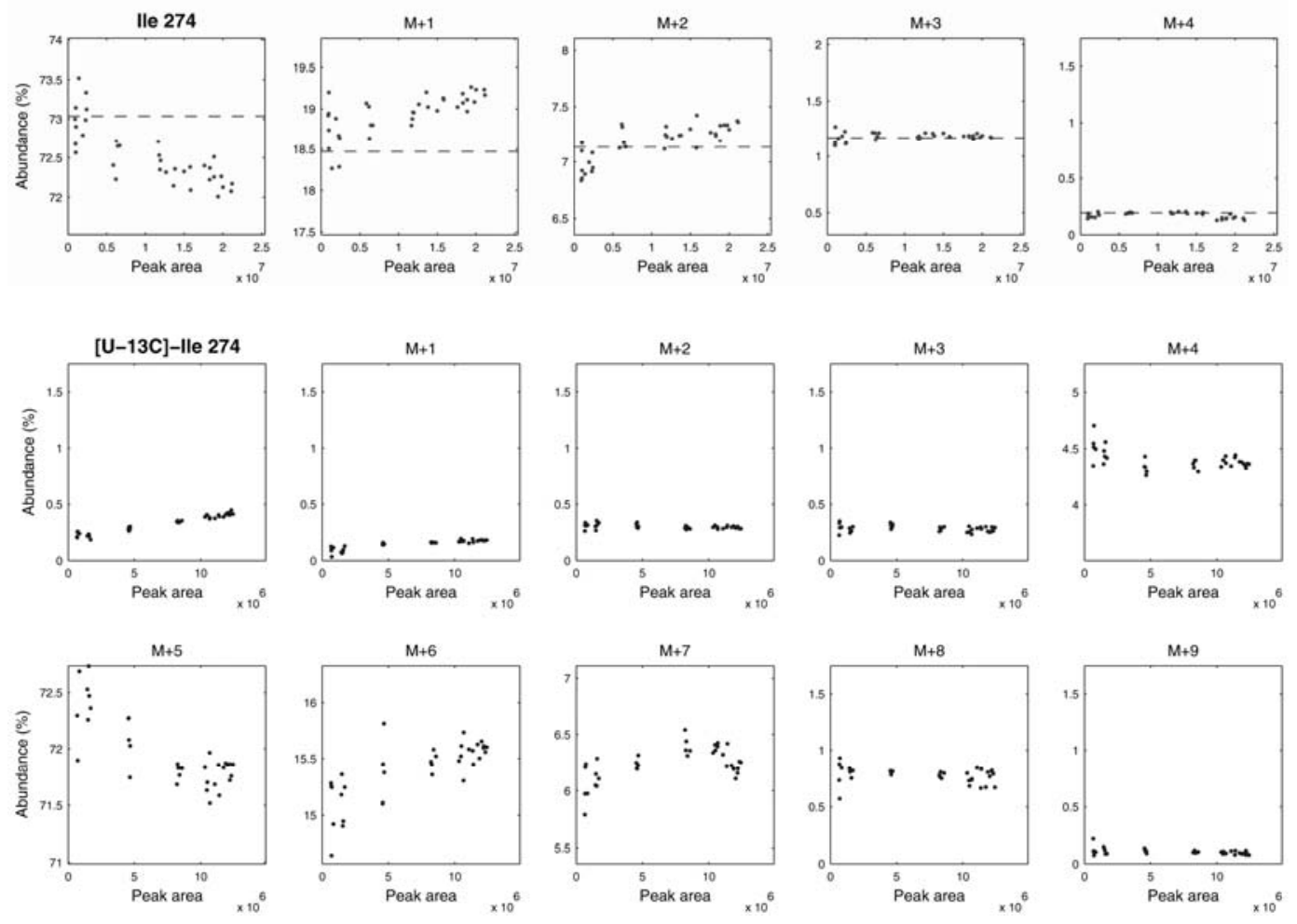

Ile 274

\begin{tabular}{lrrr}
\hline Formula : & \multicolumn{3}{c}{$\mathrm{C}_{13} \mathrm{H}_{32} \mathrm{ONSi}_{2}$} \\
Exact mass : & \multicolumn{2}{c}{274.202} \\
C-atoms : & \multicolumn{3}{c}{$2-3-4-5-6$} \\
\hline $\mathrm{m} / \mathrm{z}$ & theory & data & difference \\
\hline 273 & 0.0 & 0.0 & 0.0 \\
\hline 274 & 100.0 & 100.0 & 0.0 \\
275 & 25.3 & 25.5 & 0.2 \\
276 & 9.8 & 9.7 & -0.1 \\
277 & 1.6 & 1.6 & 0.0 \\
278 & 0.3 & 0.3 & 0.0 \\
279 & 0.0 & 0.0 & 0.0 \\
280 & 0.0 & 0.0 & 0.0 \\
281 & 0.0 & 0.0 & 0.0 \\
282 & 0.0 & 0.0 & 0.0 \\
283 & 0.0 & 0.0 & 0.0 \\
284 & 0.0 & 0.1 & 0.1 \\
\hline
\end{tabular}

$\left[\mathrm{U}-{ }^{13} \mathrm{C}\right]-\mathrm{Ile}$

\begin{tabular}{rrrr}
\hline $\mathrm{m} / \mathrm{z}$ & theory & data & difference \\
\hline 273 & 0.0 & 0.0 & 0.0 \\
\hline 274 & 0.0 & 0.3 & 0.3 \\
275 & 0.0 & 0.1 & 0.1 \\
276 & 0.0 & 0.4 & 0.4 \\
277 & 0.0 & 0.4 & 0.4 \\
278 & 5.2 & 6.1 & 0.9 \\
279 & 100.0 & 100.0 & 0.0 \\
280 & 20.1 & 20.9 & 0.8 \\
281 & 8.5 & 8.5 & 0.0 \\
282 & 1.1 & 1.1 & 0.0 \\
283 & 0.2 & 0.2 & 0.0 \\
284 & 0.0 & 0.0 & 0.0 \\
\hline
\end{tabular}

This fragment can be used for flux analysis. 

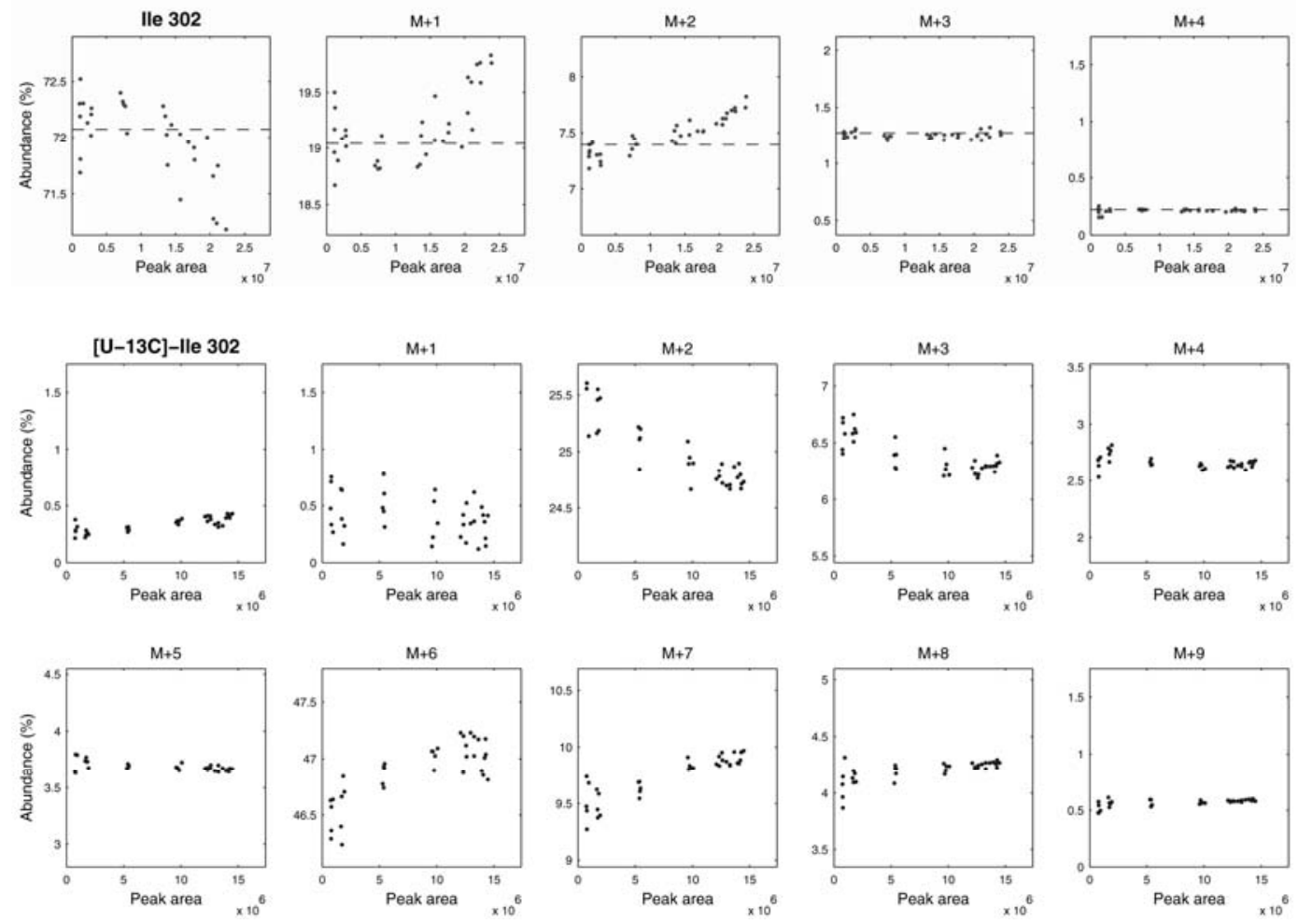

Ile 302

$\left[\mathrm{U}-{ }^{13} \mathrm{C}\right]-\mathrm{Ile}$

\begin{tabular}{|c|c|c|c|}
\hline \multicolumn{2}{|c|}{$\begin{array}{l}\text { Formula : } \\
\text { Exact mass : } \\
\text { C-atoms : }\end{array}$} & $\begin{array}{l}\mathrm{C}_{14} \mathrm{H}_{32} \mathrm{O}_{2} \mathrm{~N} \\
302.197 \\
1-2 \text { and } 1-2\end{array}$ & $-4-5-6$ \\
\hline $\mathrm{m} / \mathrm{z}$ & theory & data & difference \\
\hline 301 & 0.0 & 0.0 & 0.0 \\
\hline 302 & 100.0 & 100.0 & 0.0 \\
\hline 303 & 26.4 & 25.7 & -0.7 \\
\hline 304 & 10.3 & 10.0 & -0.3 \\
\hline 305 & 1.8 & 1.7 & -0.1 \\
\hline 306 & 0.3 & 0.3 & 0.0 \\
\hline 307 & 0.0 & 0.0 & 0.0 \\
\hline 308 & 0.0 & 0.0 & 0.0 \\
\hline 309 & 0.0 & 0.0 & 0.0 \\
\hline 310 & 0.0 & 0.0 & 0.0 \\
\hline 311 & 0.0 & 0.0 & 0.0 \\
\hline 312 & 0.0 & 0.0 & 0.0 \\
\hline 313 & 0.0 & 0.0 & 0.0 \\
\hline
\end{tabular}

\begin{tabular}{rrrr}
\hline $\mathrm{m} / \mathrm{z}$ & theory & data & difference \\
\hline 301 & 0.0 & 0.0 & 0.0 \\
\hline 302 & 0.0 & 0.5 & 0.5 \\
303 & 0.0 & 1.1 & 1.1 \\
304 & 0.0 & 54.6 & 54.6 \\
305 & 0.0 & 14.0 & 14.0 \\
306 & 0.0 & 5.8 & 5.8 \\
307 & 6.3 & 8.0 & 1.7 \\
308 & 100.0 & 100.0 & 0.0 \\
309 & 20.2 & 20.3 & 0.1 \\
310 & 8.7 & 8.8 & 0.1 \\
311 & 1.1 & 1.2 & 0.1 \\
312 & 0.2 & 0.2 & 0.0 \\
313 & 0.0 & 0.0 & 0.0 \\
\hline
\end{tabular}

This fragment should not be used for flux analysis. The presence of $\mathrm{M}+2$ and $\mathrm{M}+6$ abundances in [U${ }^{13} \mathrm{C}$ ]isoleucine suggests that two different fragments are overlapping. 


\section{Proline (Pro)}
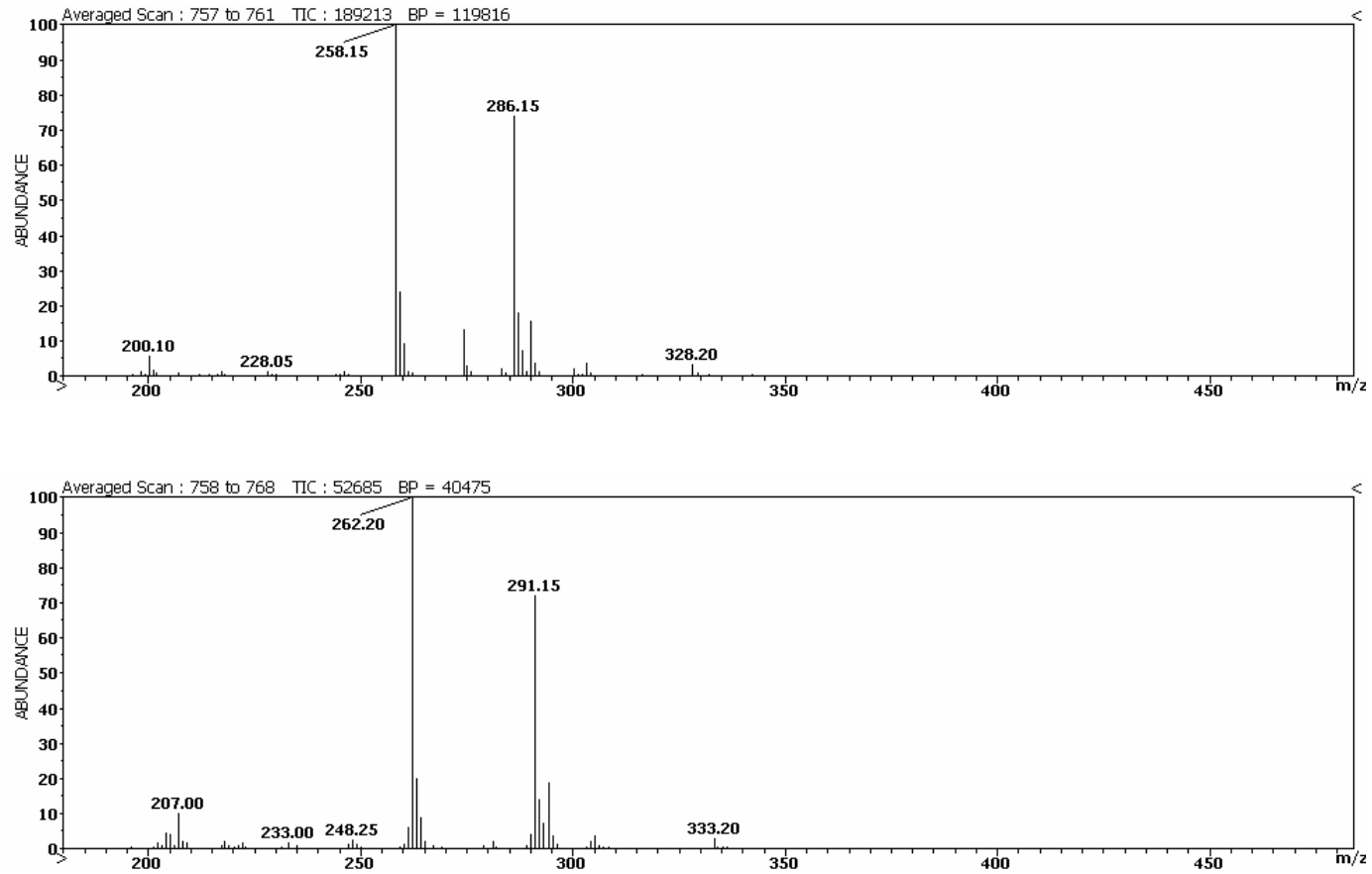<smiles>CC(C)(C)[Si](C)(C)OC(=O)C1CCCN1[Si](C)(C)C(C)(C)C</smiles>

$\mathrm{C}_{17} \mathrm{H}_{37} \mathrm{NO}_{2} \mathrm{Si}_{2}$

Exact Mass: 343.2363 

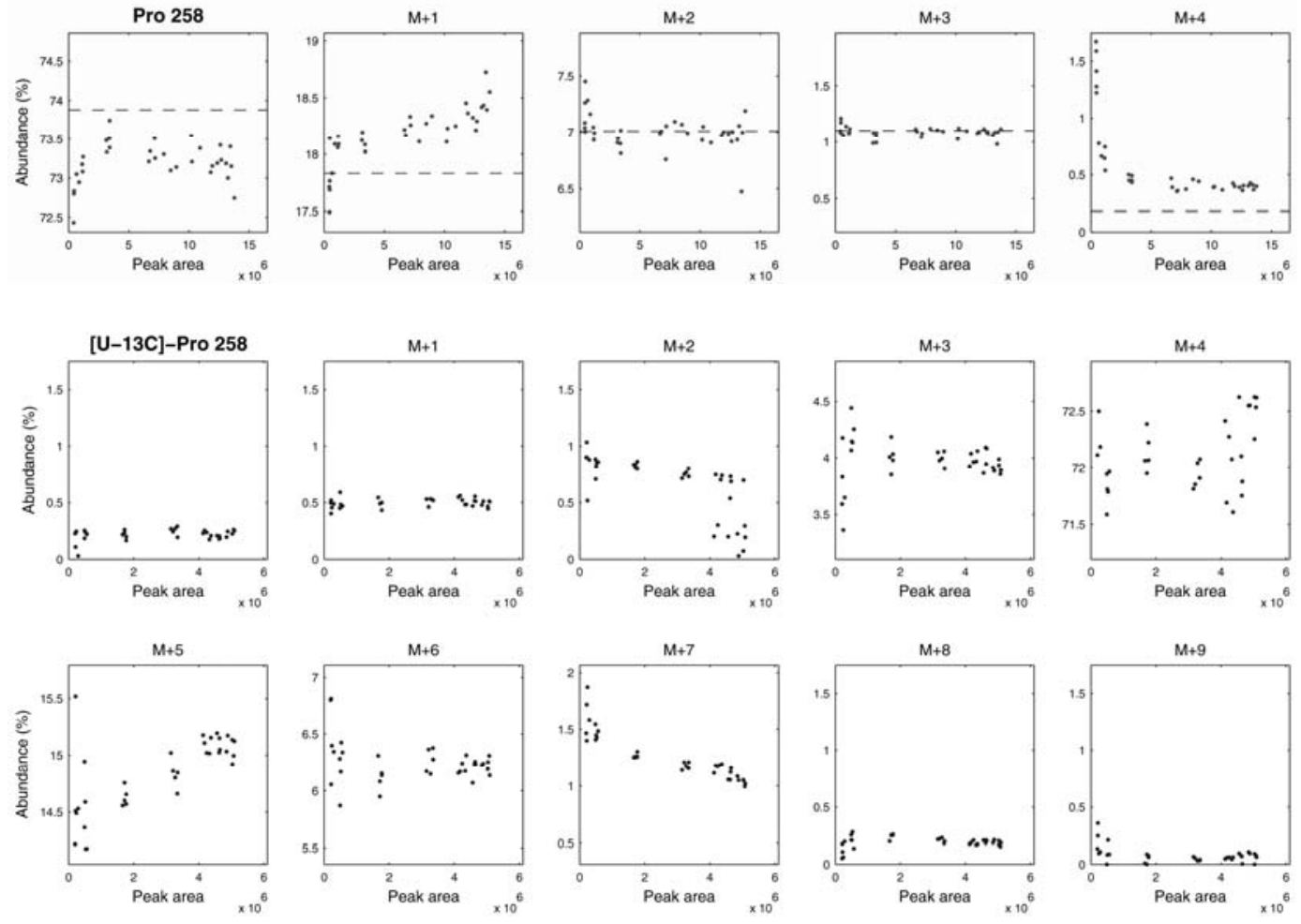

Pro 258

\begin{tabular}{lrrr}
\hline Formula : & \multicolumn{3}{c}{$\mathrm{C}_{12} \mathrm{H}_{28} \mathrm{ONSi}_{2}$} \\
Exact mass : & \multicolumn{2}{c}{258.171} \\
C-atoms : & \multicolumn{2}{c}{$2-3-4-5$} & \\
\hline $\mathrm{m} / \mathrm{z}$ & theory & data & difference \\
\hline 257 & 0.0 & 0.0 & 0.0 \\
\hline 258 & 100.0 & 100.0 & 0.0 \\
259 & 24.1 & 24.5 & 0.4 \\
260 & 9.5 & 9.5 & 0.0 \\
261 & 1.5 & 1.4 & -0.1 \\
262 & 0.2 & 0.7 & 0.5 \\
263 & 0.0 & 0.1 & 0.1 \\
264 & 0.0 & 0.1 & 0.1 \\
265 & 0.0 & 0.0 & 0.0 \\
266 & 0.0 & 0.1 & 0.1 \\
267 & 0.0 & 0.0 & 0.0 \\
\hline
\end{tabular}

\section{$\left[\mathrm{U}-{ }^{13} \mathrm{C}\right]-$ Pro}

\begin{tabular}{rrrr}
\hline $\mathrm{m} / \mathrm{z}$ & theory & data & difference \\
\hline 257 & 0.0 & 0.1 & 0.1 \\
\hline 258 & 0.0 & 0.3 & 0.3 \\
259 & 0.0 & 0.7 & 0.7 \\
260 & 0.0 & 1.5 & 1.5 \\
261 & 4.1 & 5.7 & 1.6 \\
262 & 100.0 & 100.0 & 0.0 \\
263 & 20.0 & 20.0 & 0.0 \\
264 & 8.5 & 8.6 & 0.1 \\
265 & 1.1 & 2.0 & 0.9 \\
266 & 0.2 & 0.3 & 0.1 \\
267 & 0.0 & 0.1 & 0.1 \\
\hline
\end{tabular}

This fragment should be used with caution for flux analysis. In this analysis, proline co-eluted with another compound resulting in inaccurate values, i.e. $\mathrm{M}+0$ is slightly too low $(-0.5 \mathrm{~mol} \%)$ and $\mathrm{M}+4$ is slightly too high (+0.4 mol\%). Fragment Glu-330 is preferred. 

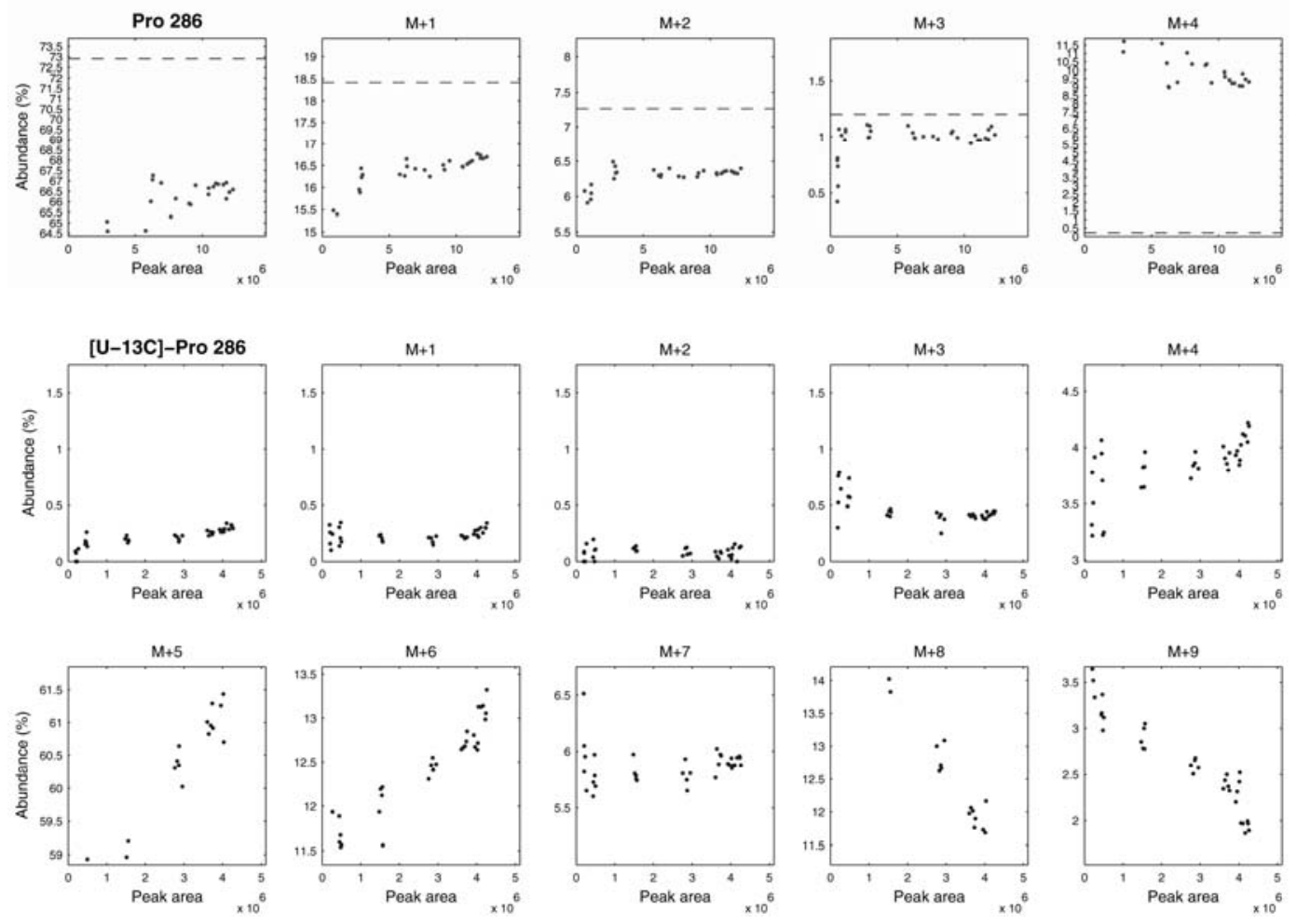

Pro 286

\begin{tabular}{lrrr}
\hline Formula : & \multicolumn{3}{c}{$\mathrm{C}_{13} \mathrm{H}_{28} \mathrm{NO}_{2} \mathrm{Si}_{2}$} \\
Exact mass : & \multicolumn{3}{c}{286.166} \\
C-atoms : & \multicolumn{2}{c}{ 1-2-3-4-5 } \\
\hline $\mathrm{m} / \mathrm{z}$ & theory & data & difference \\
\hline 285 & 0.0 & 0.0 & 0.0 \\
\hline 286 & 100.0 & 100.0 & 0.0 \\
287 & 25.3 & 25.2 & -0.1 \\
288 & 10.0 & 9.9 & -0.1 \\
289 & 1.6 & 1.7 & 0.1 \\
290 & 0.3 & 25.4 & 25.1 \\
291 & 0.0 & 6.3 & 6.3 \\
292 & 0.0 & 2.4 & 2.4 \\
293 & 0.0 & 0.5 & 0.5 \\
294 & 0.0 & 0.0 & 0.0 \\
295 & 0.0 & 0.0 & 0.0 \\
296 & 0.0 & 0.0 & 0.0 \\
297 & 0.0 & 0.0 & 0.0 \\
\hline
\end{tabular}

\section{$\left[\mathrm{U}-{ }^{13} \mathrm{C}\right]-$ Pro}

\begin{tabular}{rrrr}
\hline $\mathrm{m} / \mathrm{z}$ & theory & data & difference \\
\hline 285 & 0.0 & 0.0 & 0.0 \\
\hline 286 & 0.0 & 0.2 & 0.2 \\
287 & 0.0 & 0.3 & 0.3 \\
288 & 0.0 & 0.2 & 0.2 \\
289 & 0.0 & 0.9 & 0.9 \\
290 & 5.2 & 6.4 & 1.2 \\
291 & 100.0 & 100.0 & 0.0 \\
292 & 20.1 & 20.2 & 0.1 \\
293 & 8.7 & 10.1 & 1.4 \\
294 & 1.1 & 29.2 & 28.1 \\
295 & 0.2 & 5.9 & 5.7 \\
296 & 0.0 & 2.7 & 2.7 \\
297 & 0.0 & 0.3 & 0.3 \\
\hline
\end{tabular}

This fragment should not be used for flux analysis. In this analysis, proline co-eluted with another compound resulting in inaccurate values, i.e. $\mathrm{M}+4$ is too high $(+25 \mathrm{~mol} \%)$. Fragment Glu-432 is preferred. 


\section{Serine (Ser)}
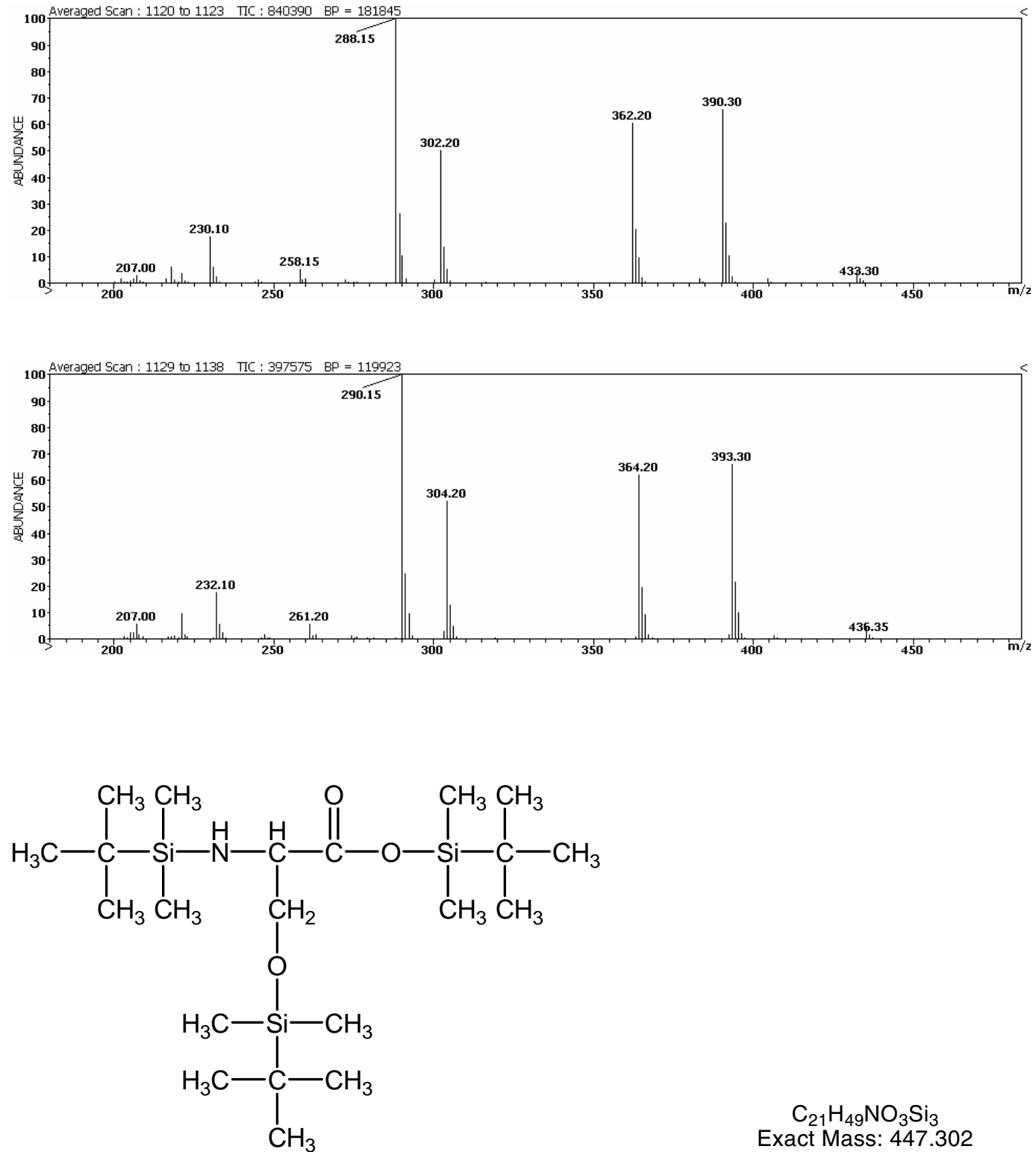

$\mathrm{C}_{21} \mathrm{H}_{49} \mathrm{NO}_{3} \mathrm{Si}_{3}$

Exact Mass: 447.302 

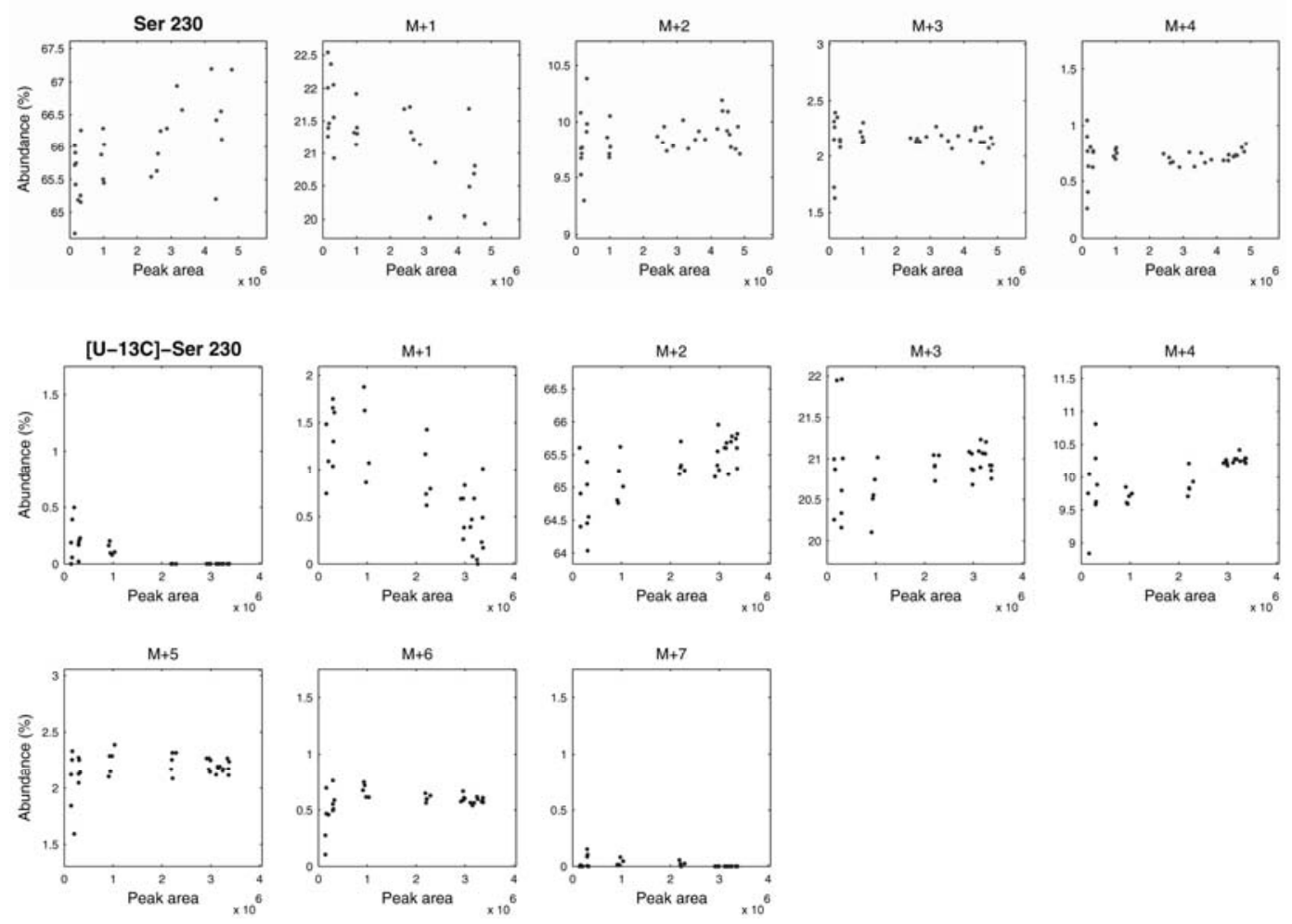

Ser 230

$\left[\mathrm{U}-{ }^{13} \mathrm{C}\right]-\mathrm{Ser}$

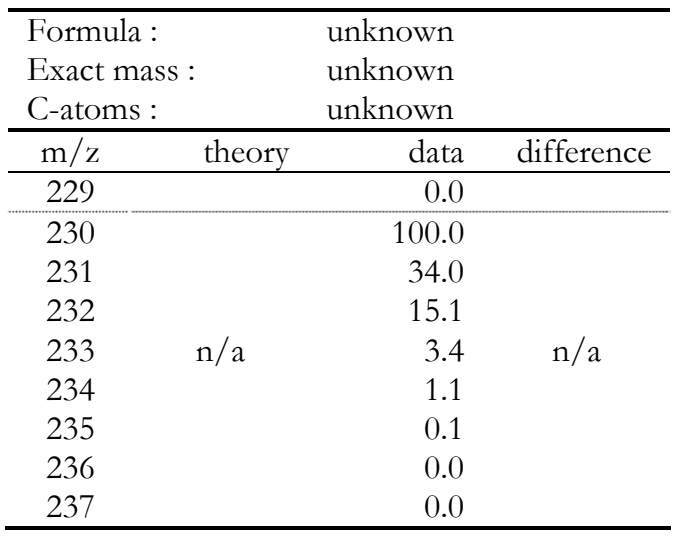

\begin{tabular}{crrr}
\hline $\mathrm{m} / \mathrm{z}$ & theory & data & difference \\
\hline 229 & & 0.0 & \\
\hline 230 & & 0.3 & \\
231 & & 2.9 & \\
232 & & 100.0 & \\
233 & $\mathrm{n} / \mathrm{a}$ & 31.8 & $\mathrm{n} / \mathrm{a}$ \\
234 & & 14.5 & \\
235 & & 3.4 & \\
236 & & 1.0 & \\
237 & & 0.1 & \\
\hline
\end{tabular}

This fragment cannot be used for flux analysis. The fragmentation is unknown. Furthermore, this fragment is not very abundant and noisy. 

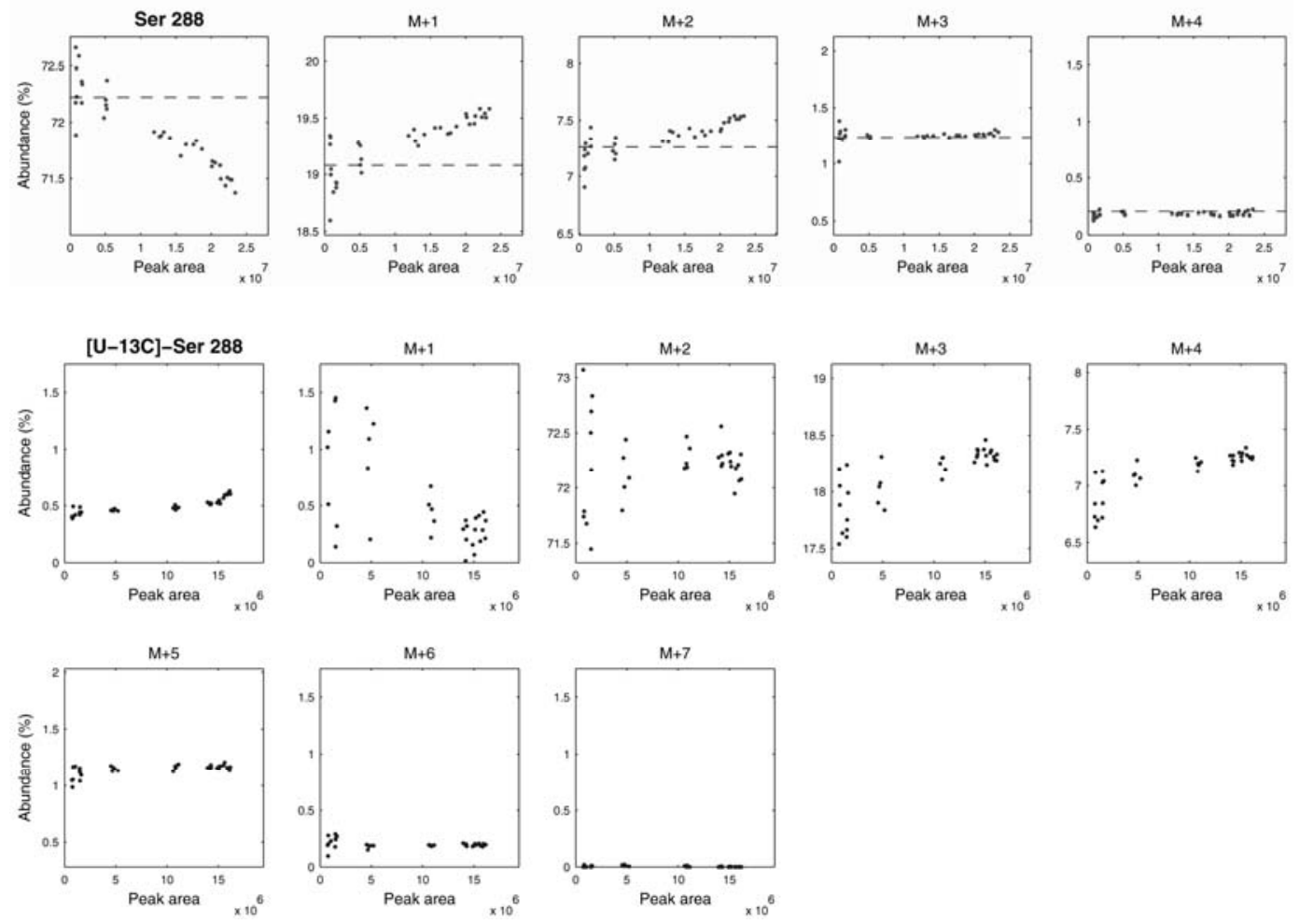

Ser 288

\begin{tabular}{lrrr}
\hline Formula : & \multicolumn{3}{l}{$\mathrm{C}_{14} \mathrm{H}_{34} \mathrm{NOSi}_{2}$} \\
Exact mass : & \multicolumn{3}{c}{288.218} \\
C-atoms : & \multicolumn{3}{c}{$2-3$} \\
\hline $\mathrm{m} / \mathrm{z}$ & theory & data & difference \\
\hline 287 & 0.0 & 0.0 & 0.0 \\
\hline 288 & 100.0 & 100.0 & 0.0 \\
289 & 26.4 & 26.3 & -0.1 \\
290 & 10.1 & 10.0 & -0.1 \\
291 & 1.7 & 1.7 & 0.0 \\
292 & 0.3 & 0.3 & 0.0 \\
293 & 0.0 & 0.0 & 0.0 \\
294 & 0.0 & 0.0 & 0.0 \\
295 & 0.0 & 0.0 & 0.0 \\
\hline
\end{tabular}

$\left[\mathrm{U}-{ }^{13} \mathrm{C}\right]-\mathrm{Ser}$

This fragment can be used for flux analysis. 

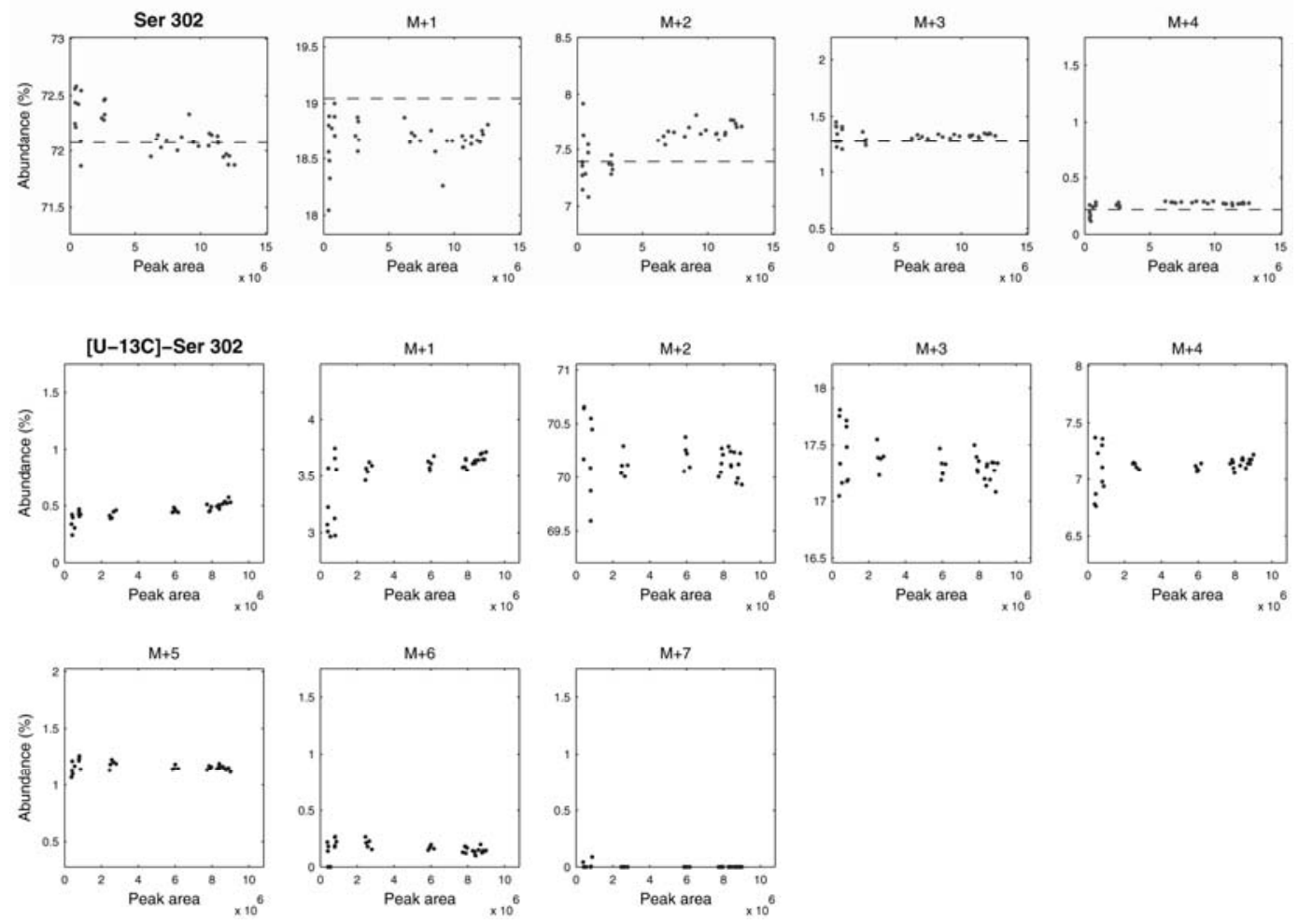

Ser 302

\begin{tabular}{lrrr}
\hline Formula : & \multicolumn{3}{c}{$\mathrm{C}_{14} \mathrm{H}_{32} \mathrm{O}_{2} \mathrm{NSi}_{2}$} \\
Exact mass : & \multicolumn{3}{c}{302.197} \\
C-atoms : & \multicolumn{3}{c}{$1-2$} \\
\hline $\mathrm{m} / \mathrm{z}$ & theory & data & difference \\
\hline 300 & 0.0 & 1.2 & 1.2 \\
301 & 0.0 & 0.0 & 0.0 \\
\hline 302 & 100.0 & 100.0 & 0.0 \\
303 & 26.4 & 26.1 & -0.3 \\
304 & 10.3 & 10.2 & -0.1 \\
305 & 1.8 & 1.8 & 0.0 \\
306 & 0.3 & 0.4 & 0.1 \\
307 & 0.0 & 0.0 & 0.0 \\
308 & 0.0 & 0.0 & 0.0 \\
309 & 0.0 & 0.0 & 0.0 \\
\hline
\end{tabular}

$\left[\mathrm{U}-{ }^{13} \mathrm{C}\right]-$ Ser

\begin{tabular}{rrrr}
\hline $\mathrm{m} / \mathrm{z}$ & theory & data & difference \\
\hline 300 & 0.0 & 0.0 & 0.0 \\
301 & 0.0 & 0.0 & 0.0 \\
\hline 302 & 0.0 & 0.5 & 0.5 \\
303 & 2.0 & 4.5 & 2.5 \\
304 & 100.0 & 100.0 & 0.0 \\
305 & 24.3 & 24.8 & 0.5 \\
306 & 9.7 & 10.1 & 0.4 \\
307 & 1.5 & 1.7 & 0.2 \\
308 & 0.3 & 0.3 & 0.0 \\
309 & 0.0 & 0.0 & 0.0 \\
\hline
\end{tabular}

This fragment should not be used for flux analysis. M-2 is too high (1.2 mol\%) resulting from an alternative fragmentation. 

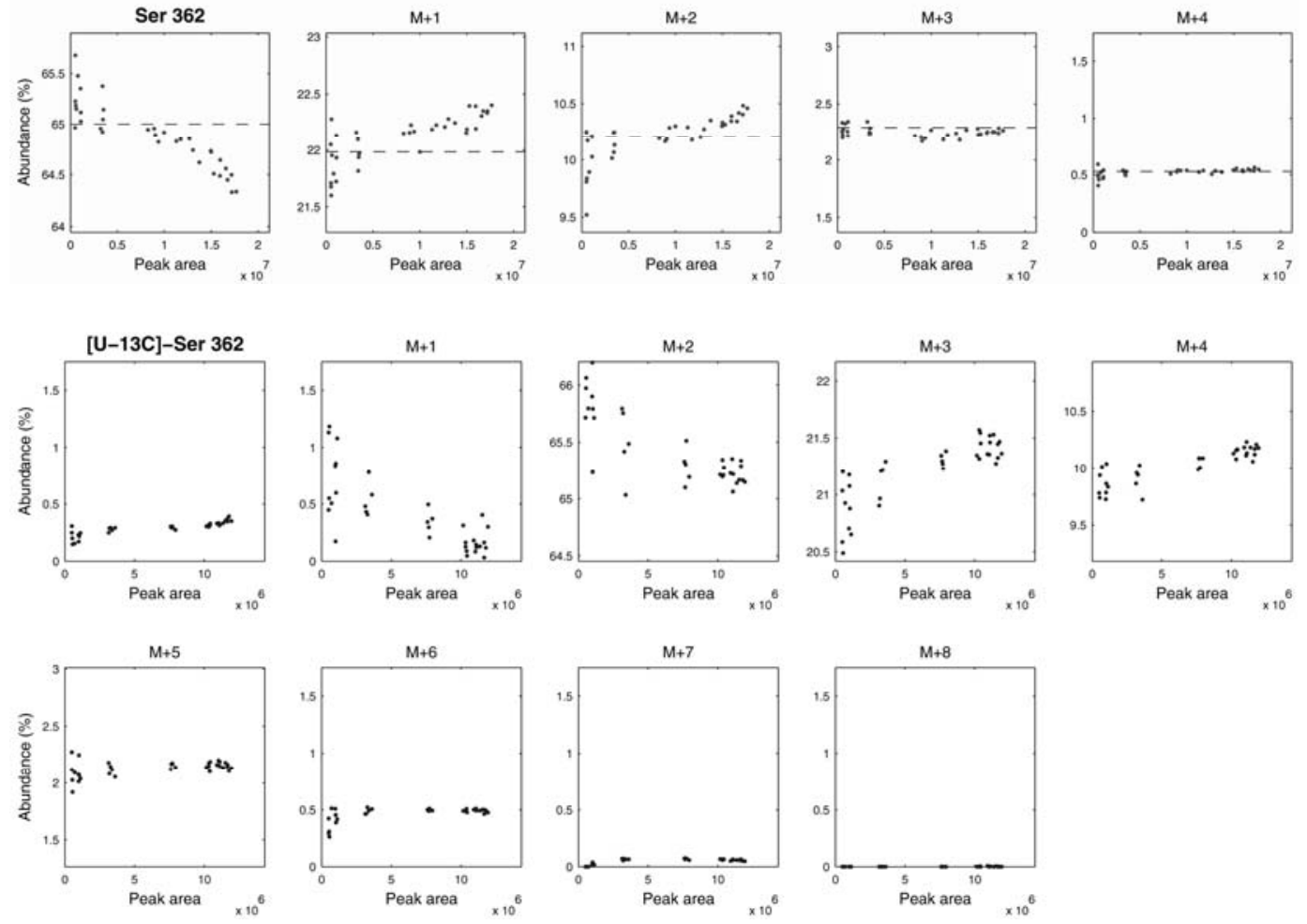

Ser 362

\begin{tabular}{lrrr}
\hline Formula : & \multicolumn{3}{l}{$\mathrm{C}_{16} \mathrm{H}_{40} \mathrm{O}_{2} \mathrm{NSi}_{3}$} \\
Exact mass : & \multicolumn{3}{c}{362.237} \\
C-atoms : & \multicolumn{3}{c}{$2-3$} \\
\hline $\mathrm{m} / \mathrm{z}$ & theory & data & difference \\
\hline 361 & 0.0 & 0.0 & 0.0 \\
\hline 362 & 100.0 & 100.0 & 0.0 \\
363 & 33.8 & 33.5 & -0.3 \\
364 & 15.7 & 15.4 & -0.3 \\
365 & 3.5 & 3.4 & -0.1 \\
366 & 0.8 & 0.8 & 0.0 \\
367 & 0.1 & 0.1 & 0.0 \\
368 & 0.0 & 0.0 & 0.0 \\
369 & 0.0 & 0.0 & 0.0 \\
370 & 0.0 & 0.0 & 0.0 \\
\hline
\end{tabular}

\section{$\left[\mathrm{U}-{ }^{13} \mathrm{C}\right]-\mathrm{Ser}$}

This fragment can be used for flux analysis.

\begin{tabular}{rrrr}
\hline $\mathrm{m} / \mathrm{z}$ & theory & data & difference \\
\hline 361 & 0.0 & 0.0 & 0.0 \\
\hline 362 & 0.0 & 0.3 & 0.3 \\
363 & 2.2 & 1.5 & -0.7 \\
364 & 100.0 & 100.0 & 0.0 \\
365 & 31.7 & 31.9 & 0.2 \\
366 & 15.0 & 14.9 & -0.1 \\
367 & 3.2 & 3.2 & 0.0 \\
368 & 0.7 & 0.7 & 0.0 \\
369 & 0.1 & 0.1 & 0.0 \\
370 & 0.0 & 0.0 & 0.0 \\
\hline
\end{tabular}



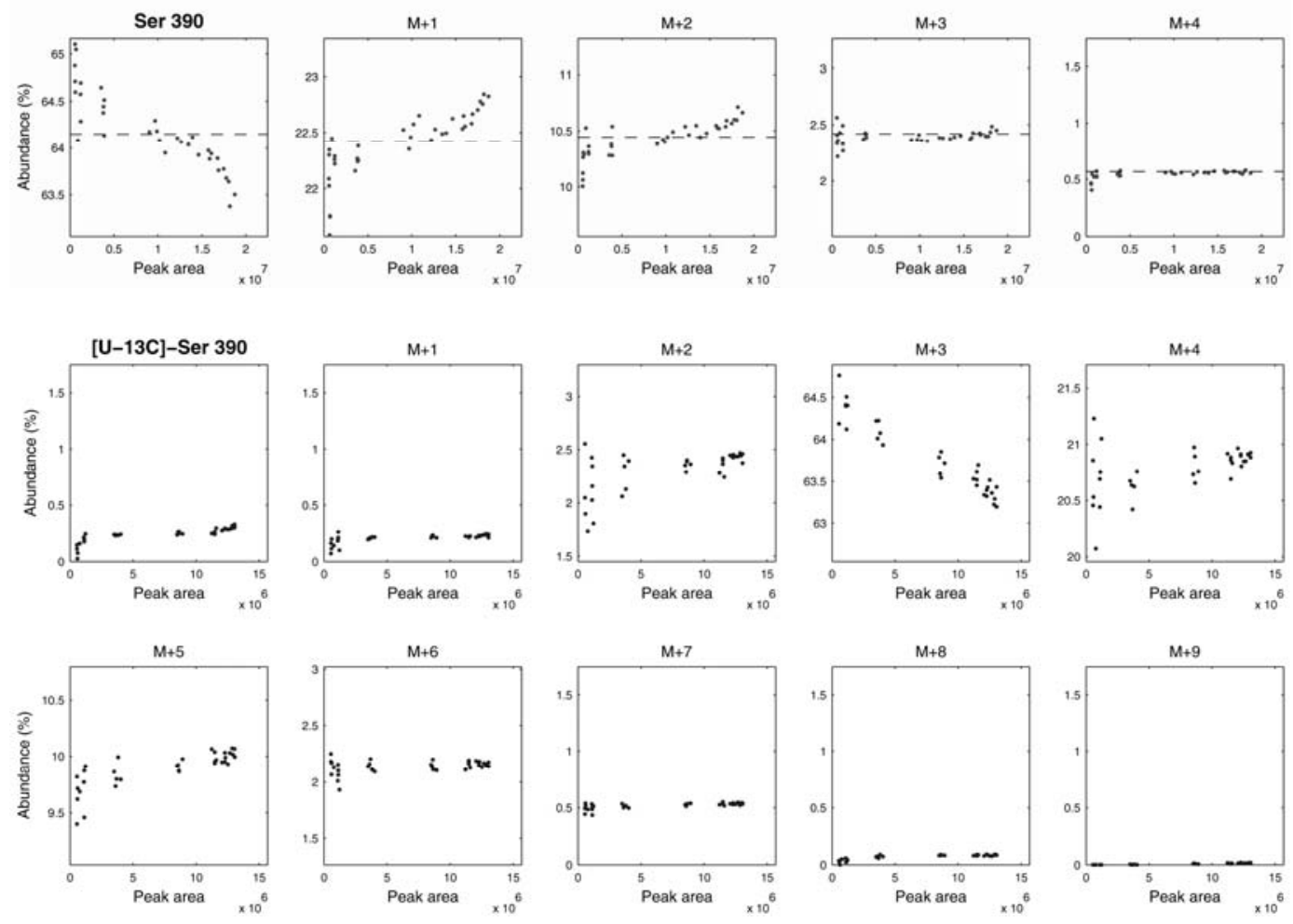

Ser 390

\section{$\left[\mathrm{U}-{ }^{13} \mathrm{C}\right]-\mathrm{Ser}$}

\begin{tabular}{|c|c|c|c|}
\hline \multicolumn{2}{|c|}{$\begin{array}{l}\text { Formula : } \\
\text { Exact mass : } \\
\text { C-atoms : }\end{array}$} & \multicolumn{2}{|c|}{$\begin{array}{l}\mathrm{C}_{17} \mathrm{H}_{40} \mathrm{O}_{3} \mathrm{NSi}_{3} \\
390.232 \\
1-2-3\end{array}$} \\
\hline $\mathrm{m} / \mathrm{z}$ & theory & data & difference \\
\hline 389 & 0.0 & 0.0 & 0.0 \\
\hline 390 & 100.0 & 100.0 & 0.0 \\
\hline 391 & 35.0 & 34.4 & -0.6 \\
\hline 392 & 16.3 & 15.9 & -0.4 \\
\hline 393 & 3.8 & 3.7 & -0.1 \\
\hline 394 & 0.9 & 0.8 & -0.1 \\
\hline 395 & 0.1 & 0.1 & 0.0 \\
\hline 396 & 0.0 & 0.0 & 0.0 \\
\hline 397 & 0.0 & 0.1 & 0.1 \\
\hline 398 & 0.0 & 0.0 & 0.0 \\
\hline 399 & 0.0 & 0.0 & 0.0 \\
\hline
\end{tabular}

\begin{tabular}{rrrr}
\hline $\mathrm{m} / \mathrm{z}$ & theory & data & difference \\
\hline 389 & 0.0 & 0.0 & 0.0 \\
\hline 390 & 0.0 & 0.3 & 0.3 \\
391 & 0.0 & 0.3 & 0.3 \\
392 & 3.1 & 3.3 & 0.2 \\
393 & 100.0 & 100.0 & 0.0 \\
394 & 31.8 & 31.9 & 0.1 \\
395 & 15.2 & 15.1 & -0.1 \\
396 & 3.3 & 3.3 & 0.0 \\
397 & 0.8 & 0.8 & 0.0 \\
398 & 0.1 & 0.1 & 0.0 \\
399 & 0.0 & 0.0 & 0.0 \\
\hline
\end{tabular}

This fragment can be used for flux analysis (with caution). Note that $\mathrm{M}+0$ is slightly too high $(+0.5$ $\mathrm{mol} \%)$ and $\mathrm{M}+1$ is slightly too low $(-0.4 \mathrm{~mol} \%)$, and that the data is very noisy at low concentrations. 


\section{Threonine (Thr)}
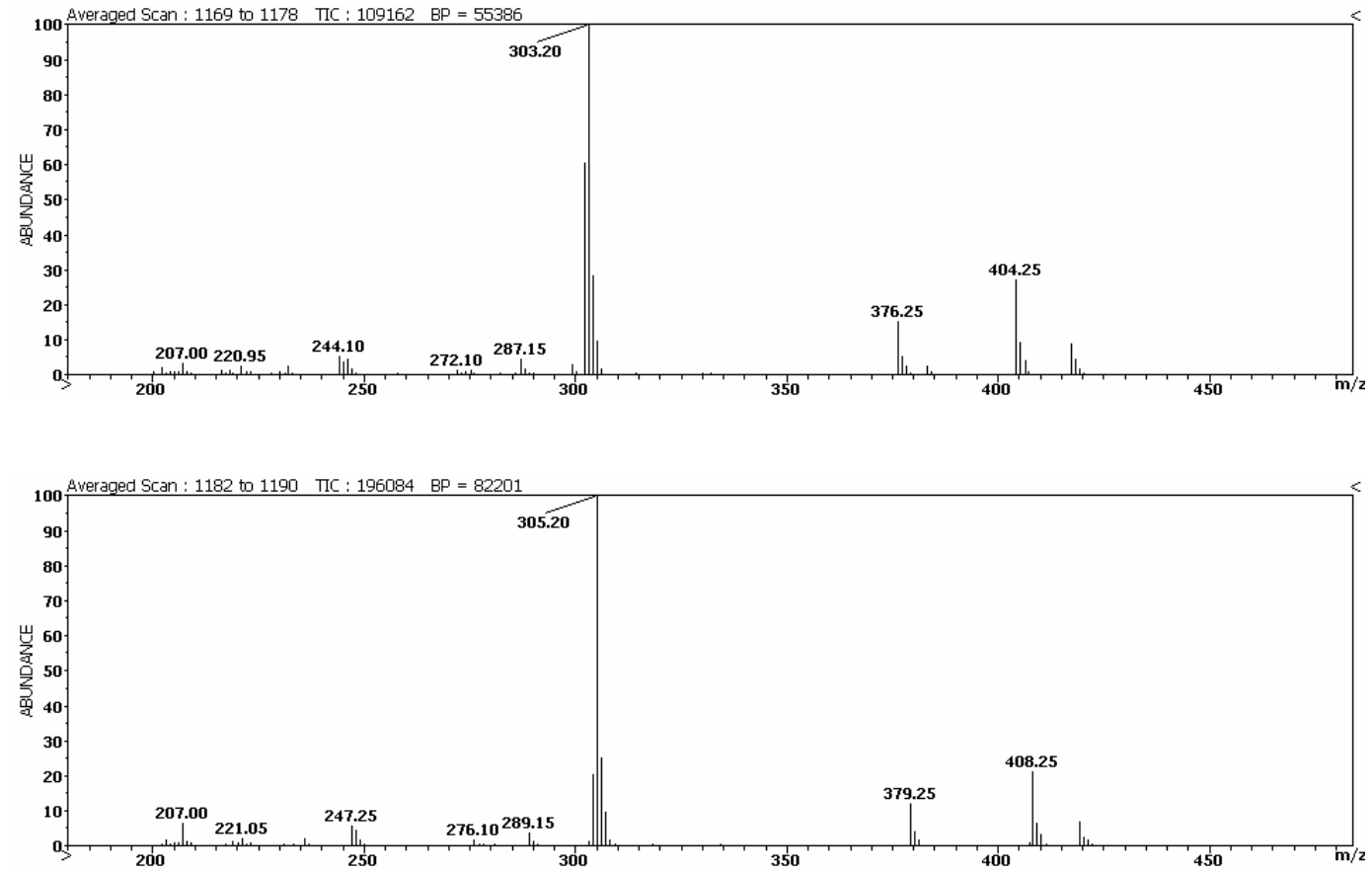<smiles>CC(O[Si](C)(C)C(C)(C)C)C(N[Si](C)(C)C(C)(C)C)C(=O)O[Si](C)(C)C(C)(C)C</smiles>

$\mathrm{C}_{22} \mathrm{H}_{51} \mathrm{NO}_{3} \mathrm{Si}_{3}$ Exact Mass: 461.3177 

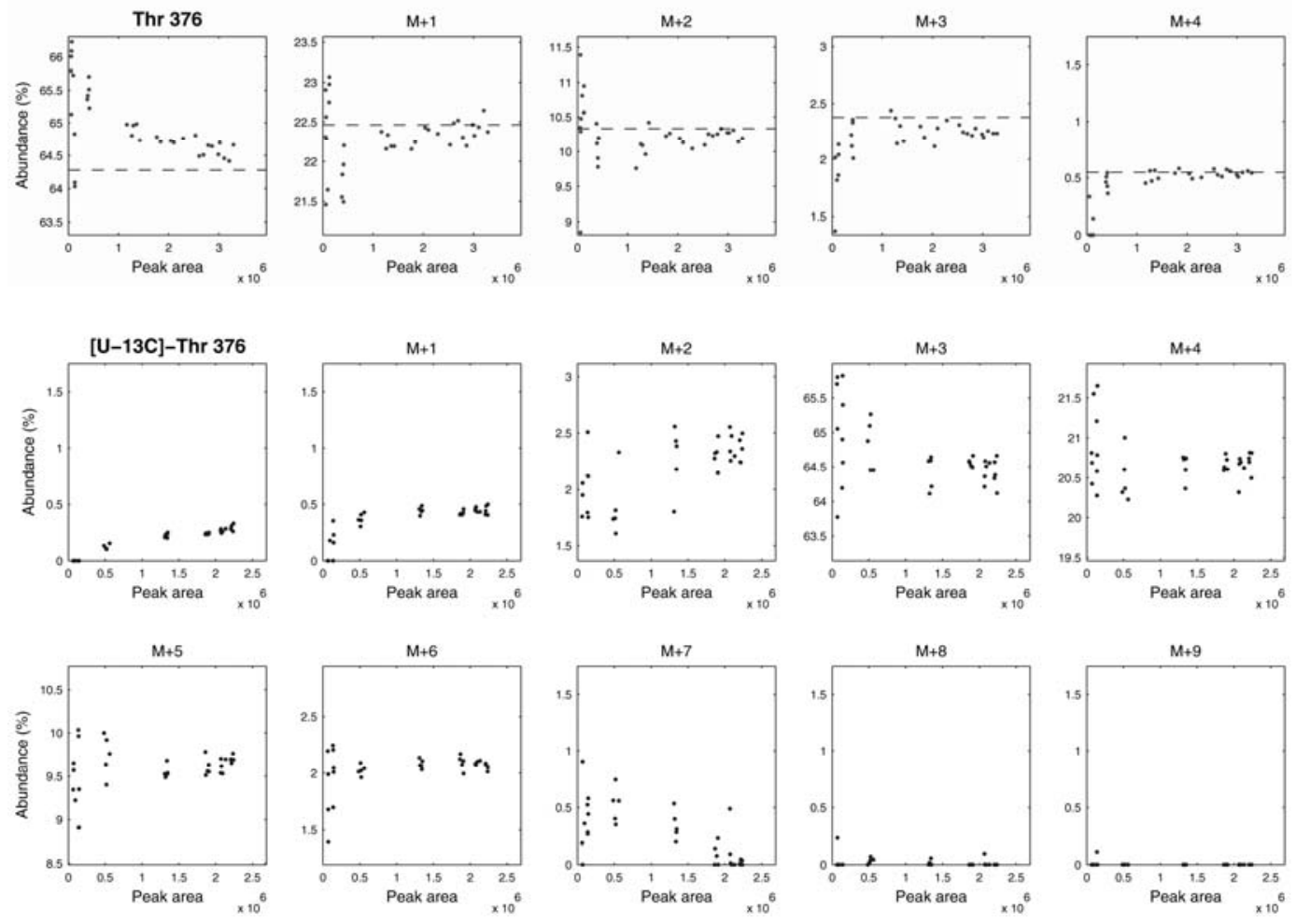

Thr 376

\begin{tabular}{lrrr}
\hline Formula : & \multicolumn{3}{c}{$\mathrm{C}_{17} \mathrm{H}_{42} \mathrm{O}_{2} \mathrm{NSi}_{3}$} \\
Exact mass : & \multicolumn{3}{c}{ 2-3-4 } \\
C-atoms : & \multicolumn{3}{c}{ - } \\
\hline $\mathrm{m} / \mathrm{z}$ & theory & data & difference \\
\hline 375 & 0.0 & 0.0 & 0.0 \\
\hline 376 & 100.0 & 100.0 & 0.0 \\
377 & 34.9 & 34.5 & -0.4 \\
378 & 16.1 & 15.9 & -0.2 \\
379 & 3.7 & 3.7 & 0.0 \\
380 & 0.9 & 0.7 & -0.2 \\
381 & 0.1 & 0.0 & -0.1 \\
382 & 0.0 & 0.0 & 0.0 \\
383 & 0.0 & 0.2 & 0.2 \\
384 & 0.0 & 0.6 & 0.6 \\
385 & 0.0 & 0.0 & 0.0 \\
\hline
\end{tabular}

$\left[\mathrm{U}-{ }^{13} \mathrm{C}\right]-\mathrm{Thr}$

This fragment can be used for flux analysis (with caution). Note that this fragment is very noisy at low concentrations, and thus should be measured at higher concentrations only. 

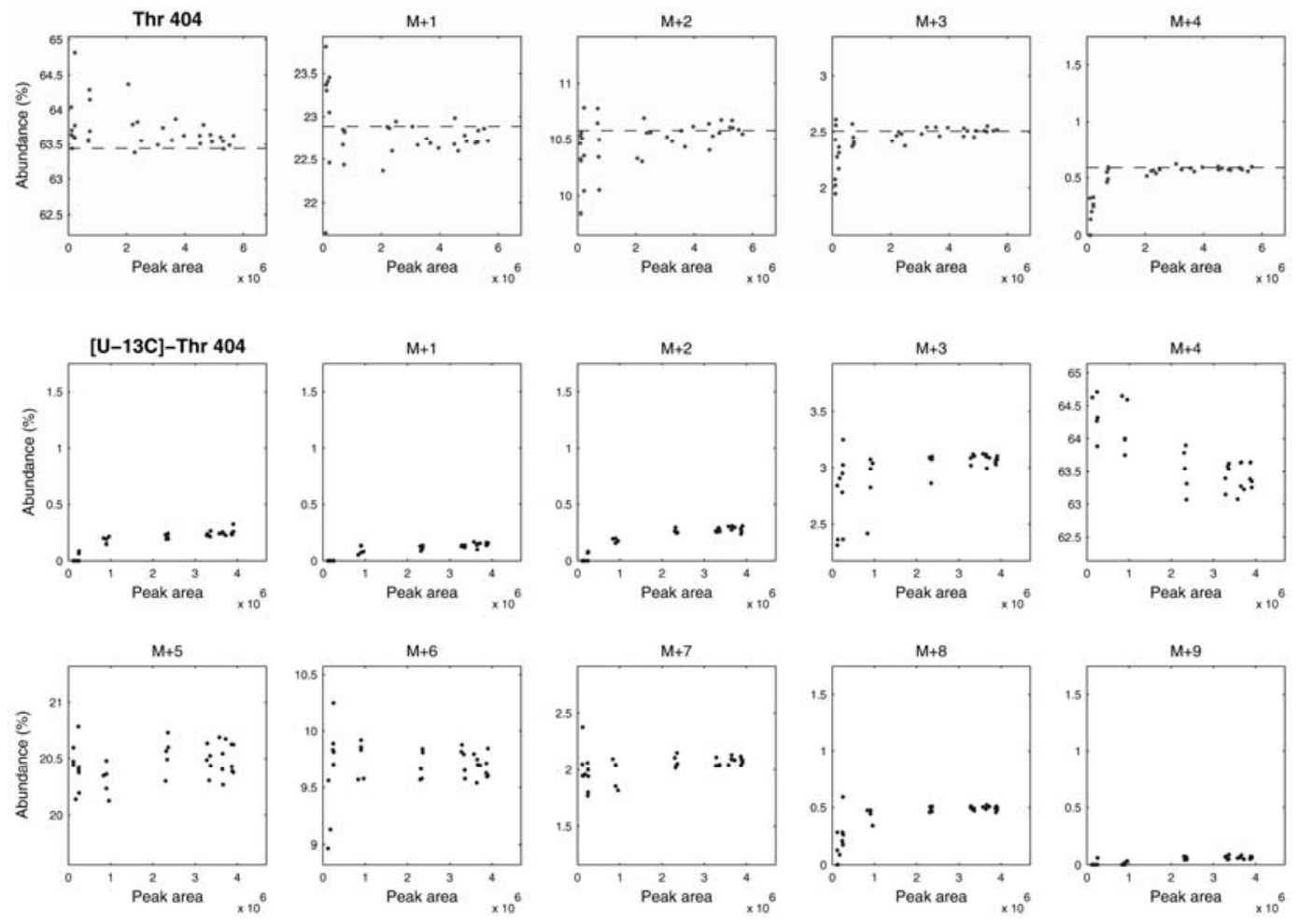

Thr 404

\begin{tabular}{lrrr}
\hline Formula : & \multicolumn{3}{c}{$\mathrm{C}_{18} \mathrm{H}_{42} \mathrm{O}_{3} \mathrm{NSi}_{3}$} \\
Exact mass : & \multicolumn{3}{c}{404.247} \\
C-atoms : & \multicolumn{3}{c}{$1-2-3-4$} \\
\hline $\mathrm{m} / \mathrm{z}$ & theory & data & difference \\
\hline 403 & 0.0 & 0.0 & 0.0 \\
\hline 404 & 100.0 & 100.0 & 0.0 \\
405 & 36.1 & 35.8 & -0.3 \\
406 & 16.7 & 16.4 & -0.3 \\
407 & 4.0 & 3.8 & -0.2 \\
408 & 0.9 & 0.8 & -0.1 \\
409 & 0.2 & 0.1 & -0.1 \\
410 & 0.0 & 0.0 & 0.0 \\
411 & 0.0 & 0.0 & 0.0 \\
412 & 0.0 & 0.0 & 0.0 \\
413 & 0.0 & 0.0 & 0.0 \\
414 & 0.0 & 0.0 & 0.0 \\
\hline
\end{tabular}

\section{$\left[\mathrm{U}-{ }^{13} \mathrm{C}\right]-\mathrm{Thr}$}

\begin{tabular}{rrrr}
\hline $\mathrm{m} / \mathrm{z}$ & theory & data & difference \\
\hline 403 & 0.0 & 0.0 & 0.0 \\
\hline 404 & 0.0 & 0.2 & 0.2 \\
405 & 0.0 & 0.1 & 0.1 \\
406 & 0.0 & 0.2 & 0.2 \\
407 & 4.1 & 4.4 & 0.3 \\
408 & 100.0 & 100.0 & 0.0 \\
409 & 31.9 & 31.6 & -0.3 \\
410 & 15.2 & 15.2 & 0.0 \\
411 & 3.3 & 3.0 & -0.3 \\
412 & 0.8 & 0.6 & -0.2 \\
413 & 0.1 & 0.0 & -0.1 \\
414 & 0.0 & 0.0 & 0.0 \\
\hline
\end{tabular}

This fragment can be used for flux analysis. 

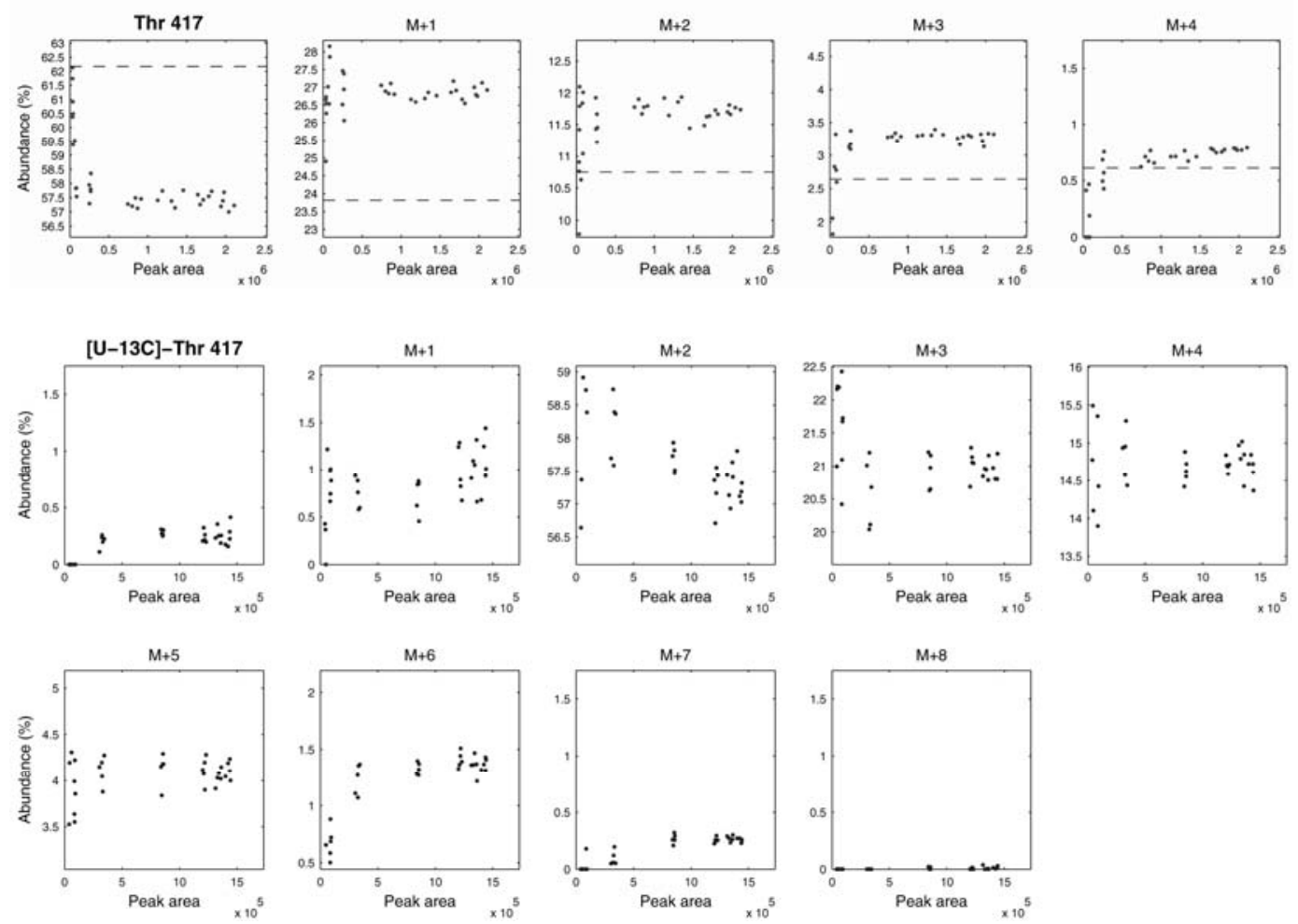

Thr 417

\begin{tabular}{lrrr}
\hline Formula : & \multicolumn{3}{c}{$\mathrm{C}_{20} \mathrm{H}_{47} \mathrm{NO}_{2} \mathrm{Si}_{3}$} \\
Exact mass : & \multicolumn{3}{c}{417.292} \\
C-atoms : & \multicolumn{3}{c}{ unknown } \\
\hline $\mathrm{m} / \mathrm{z}$ & theory & data & difference \\
\hline 416 & 0.0 & 0.0 & 0.0 \\
\hline 417 & 100.0 & 100.0 & 0.0 \\
418 & 38.3 & 46.6 & 8.3 \\
419 & 17.3 & 20.3 & 3.0 \\
420 & 4.3 & 5.6 & 1.3 \\
421 & 1.0 & 1.0 & 0.0 \\
422 & 0.2 & 0.0 & -0.2 \\
423 & 0.0 & 0.0 & 0.0 \\
424 & 0.0 & 0.0 & 0.0 \\
425 & 0.0 & 0.2 & 0.2 \\
\hline
\end{tabular}

$\left[\mathrm{U}-{ }^{13} \mathrm{C}\right]-\mathrm{Thr}$

\begin{tabular}{rrrr}
\hline $\mathrm{m} / \mathrm{z}$ & theory & data & difference \\
\hline 416 & 0.0 & 0.0 & 0.0 \\
\hline 417 & 0.0 & 0.3 & 0.3 \\
418 & 2.0 & 1.1 & -0.9 \\
419 & 100.0 & 100.0 & 0.0 \\
420 & 36.2 & 35.5 & -0.7 \\
421 & 16.4 & 25.0 & 8.6 \\
422 & 3.9 & 6.9 & 3.0 \\
423 & 0.9 & 1.8 & 0.9 \\
424 & 0.1 & 0.1 & 0.0 \\
425 & 0.0 & 0.0 & 0.0 \\
\hline
\end{tabular}

This fragment should not be used for flux analysis. This fragment has a significant bias, i.e. $\mathrm{M}+0$ is too low $(-4 \mathrm{~mol} \%)$ and $\mathrm{M}+1, \mathrm{M}+2$, and $\mathrm{M}+3$ are too high. 


\section{Methionine (Met)}
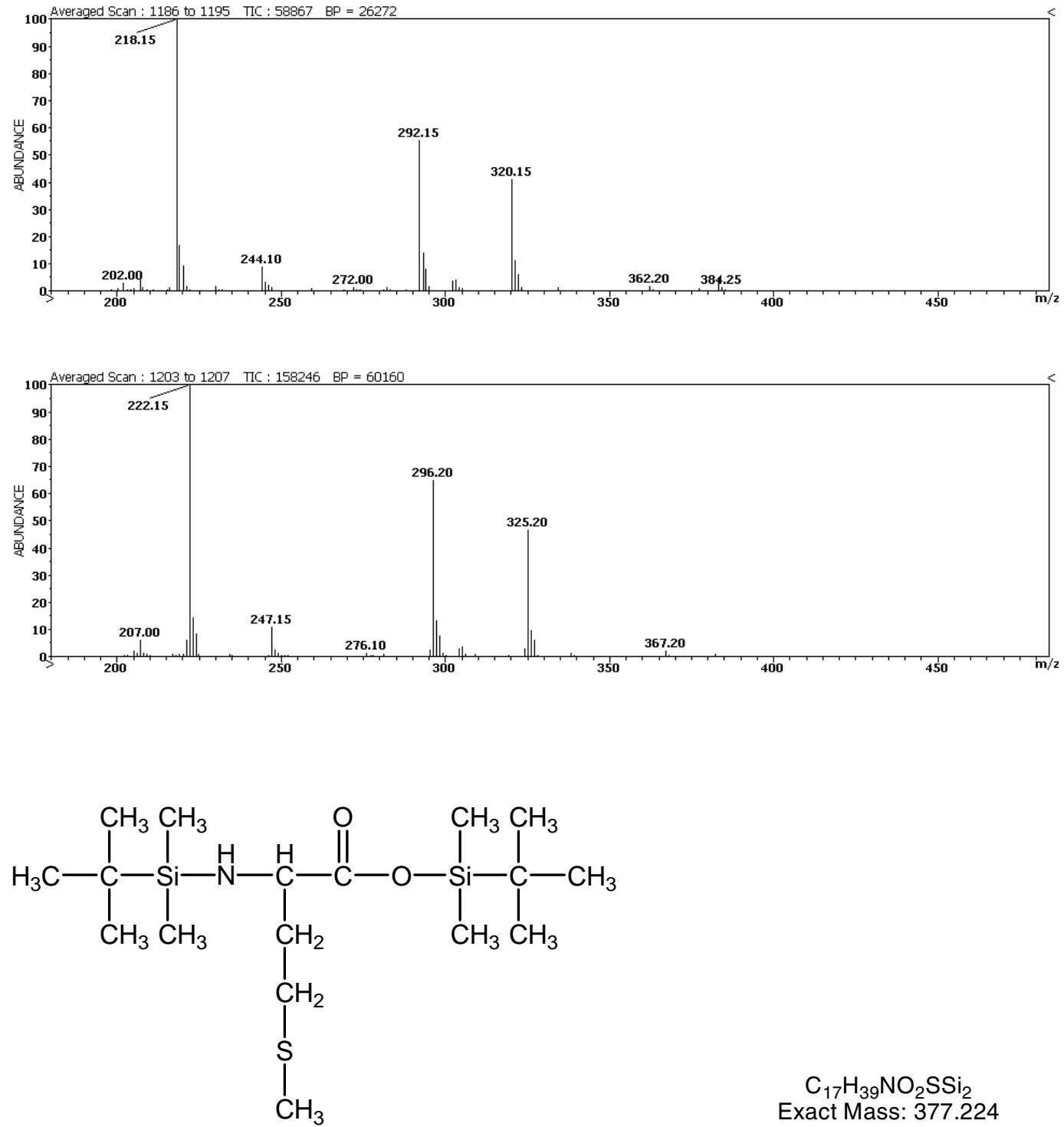

$$
\mathrm{C}_{17} \mathrm{H}_{39} \mathrm{NO}_{2} \mathrm{SSi}_{2}
$$

Exact Mass: 377.224 

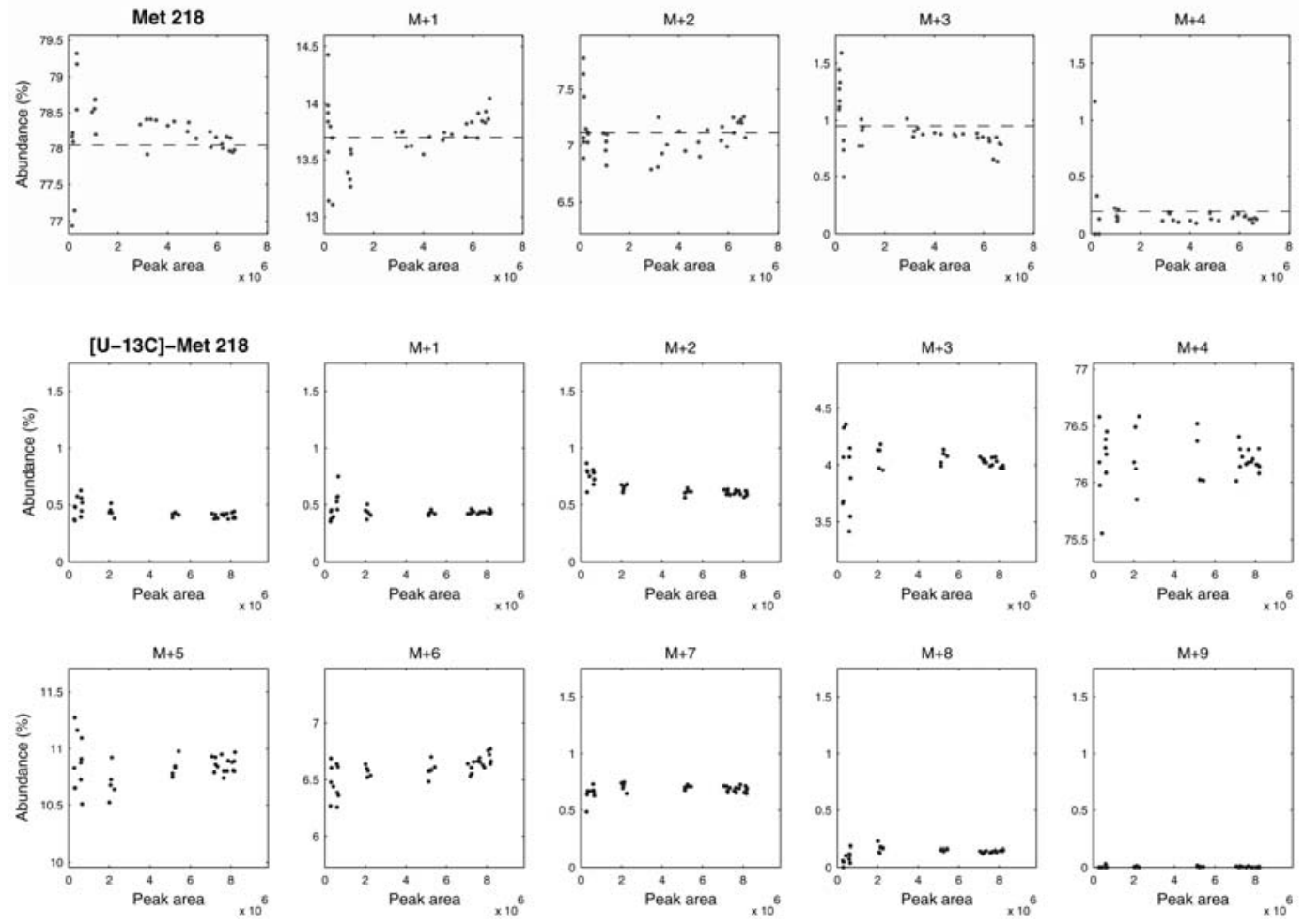

Met 218

\begin{tabular}{lrrr}
\hline Formula : & \multicolumn{3}{c}{$\mathrm{C}_{10} \mathrm{H}_{24} \mathrm{NSiS}$} \\
Exact mass : & \multicolumn{2}{c}{218.114} \\
C-atoms : & \multicolumn{2}{c}{$2-3-4-5$} & \\
\hline $\mathrm{m} / \mathrm{z}$ & theory & data & difference \\
\hline 217 & 0.0 & 0.0 & 0.0 \\
\hline 218 & 100.0 & 100.0 & 0.0 \\
219 & 17.5 & 17.3 & -0.2 \\
220 & 9.1 & 9.0 & -0.1 \\
221 & 1.2 & 1.2 & 0.0 \\
222 & 0.2 & 0.2 & 0.0 \\
223 & 0.0 & 0.0 & 0.0 \\
224 & 0.0 & 0.0 & 0.0 \\
225 & 0.0 & 0.0 & 0.0 \\
226 & 0.0 & 0.0 & 0.0 \\
227 & 0.0 & 0.0 & 0.0 \\
\hline
\end{tabular}

$\underline{\left[\mathrm{U}-{ }^{13} \mathrm{C}\right]-\mathrm{Met}}$

\begin{tabular}{rrrr}
\hline $\mathrm{m} / \mathrm{z}$ & theory & data & difference \\
\hline 217 & 0.0 & 0.7 & 0.7 \\
\hline 218 & 0.0 & 0.6 & 0.6 \\
219 & 0.0 & 0.6 & 0.6 \\
220 & 0.0 & 0.9 & 0.9 \\
221 & 4.1 & 5.3 & 1.2 \\
222 & 100.0 & 100.0 & 0.0 \\
223 & 13.5 & 14.1 & 0.6 \\
224 & 8.5 & 8.5 & 0.0 \\
225 & 0.8 & 0.9 & 0.1 \\
226 & 0.2 & 0.2 & 0.0 \\
227 & 0.0 & 0.0 & 0.0 \\
\hline
\end{tabular}

This fragment can be used for flux analysis. Note that this fragment is noisy at low concentrations, and thus should be measured at higher concentrations only. 

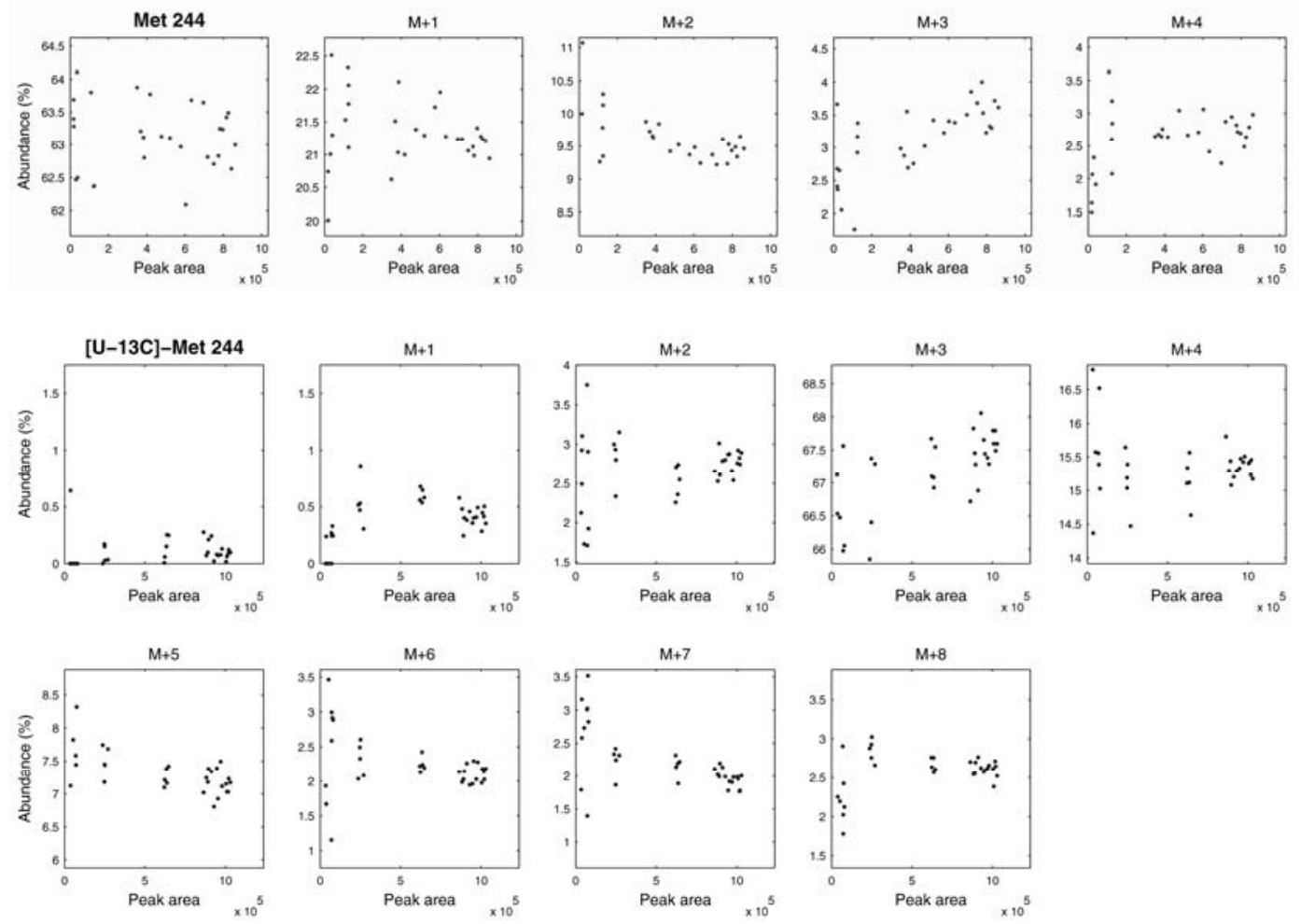

Met 244

$\left[\mathrm{U}-{ }^{13} \mathrm{C}\right]-\mathrm{Met}$

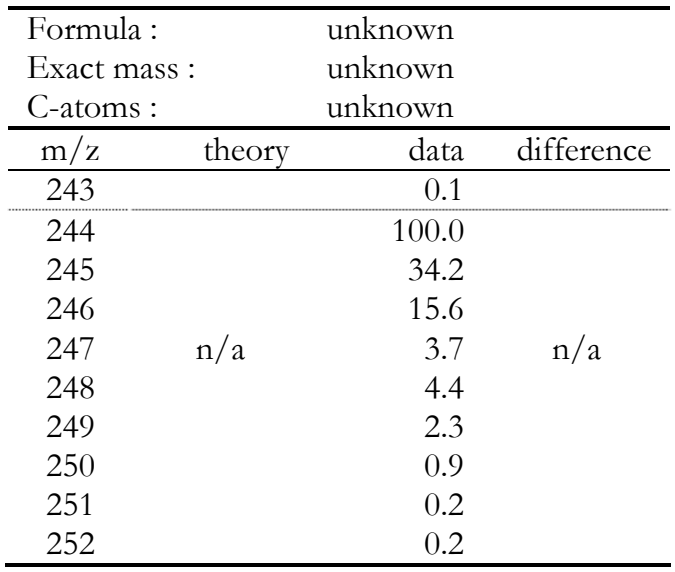

\begin{tabular}{ccrc}
\hline $\mathrm{m} / \mathrm{z}$ & theory & data & difference \\
\hline 243 & & 0.1 & \\
\hline 244 & & 0.2 & \\
245 & & 0.8 & \\
246 & & 3.9 & \\
247 & $\mathrm{n} / \mathrm{a}$ & 100.0 & $\mathrm{n} / \mathrm{a}$ \\
248 & & 23.1 & \\
249 & & 11.8 & \\
250 & & 3.8 & \\
251 & & 3.8 & \\
252 & & 4.1 & \\
\hline
\end{tabular}

This fragment cannot be used for flux analysis. The fragmentation is unknown. Furthermore, the relatively high $\mathrm{M}+1(34 \%$ of $\mathrm{M}+0)$ suggests that multiple fragments may be overlapping. 

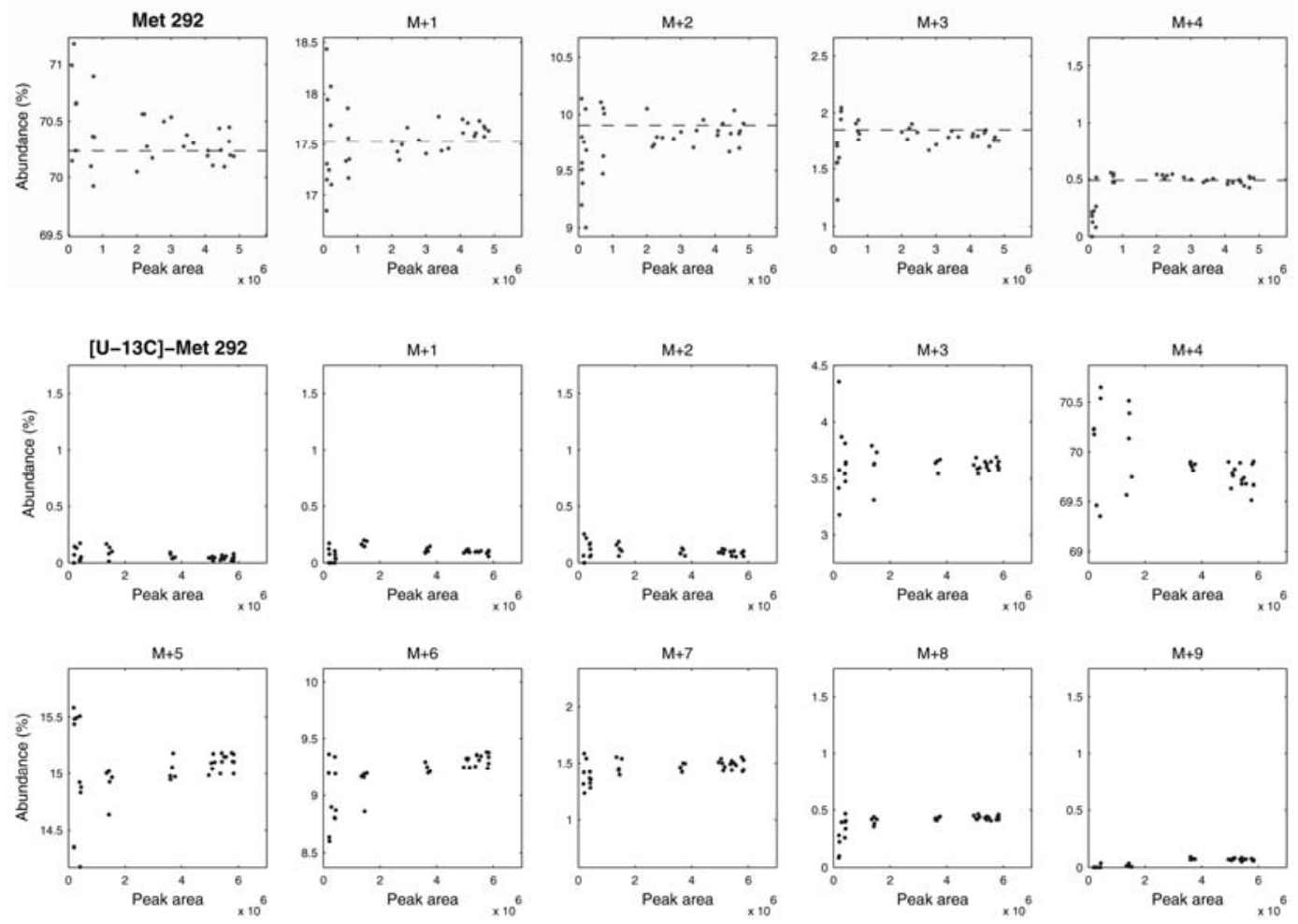

Met 292

\begin{tabular}{lrrr}
\hline Formula : & \multicolumn{3}{c}{$\mathrm{C}_{12} \mathrm{H}_{30} \mathrm{NOSi} 2 \mathrm{~S}$} \\
Exact mass : & \multicolumn{2}{c}{292.159} \\
C-atoms : & \multicolumn{2}{c}{$2-3-4-5$} & \\
\hline $\mathrm{m} / \mathrm{z}$ & theory & data & difference \\
\hline 291 & 0.0 & 0.0 & 0.0 \\
\hline 292 & 100.0 & 100.0 & 0.0 \\
293 & 25.0 & 24.8 & -0.2 \\
294 & 14.1 & 14.0 & -0.1 \\
295 & 2.6 & 2.6 & 0.0 \\
296 & 0.7 & 0.8 & 0.1 \\
297 & 0.1 & 0.1 & 0.0 \\
298 & 0.0 & 0.0 & 0.0 \\
299 & 0.0 & 3.3 & 3.3 \\
300 & 0.0 & 0.2 & 0.2 \\
301 & 0.0 & 0.3 & 0.3 \\
\hline
\end{tabular}

$\underline{\left[\mathrm{U}-{ }^{13} \mathrm{C}\right]-\mathrm{Met}}$

\begin{tabular}{crrr}
\hline $\mathrm{m} / \mathrm{z}$ & theory & data & difference \\
\hline 291 & 0.0 & 0.0 & 0.0 \\
\hline 292 & 0.0 & 0.1 & 0.1 \\
293 & 0.0 & 0.2 & 0.2 \\
294 & 0.0 & 0.2 & 0.2 \\
295 & 4.1 & 5.1 & 1.0 \\
296 & 100.0 & 100.0 & 0.0 \\
297 & 20.9 & 21.1 & 0.2 \\
298 & 13.1 & 12.8 & -0.3 \\
299 & 2.1 & 2.0 & -0.1 \\
300 & 0.6 & 0.5 & -0.1 \\
301 & 0.1 & 0.0 & -0.1 \\
\hline
\end{tabular}

This fragment can be used for flux analysis. Mass isotopomer distributions should be obtained from the mass range $\mathrm{m} / \mathrm{z} 292-298$. 

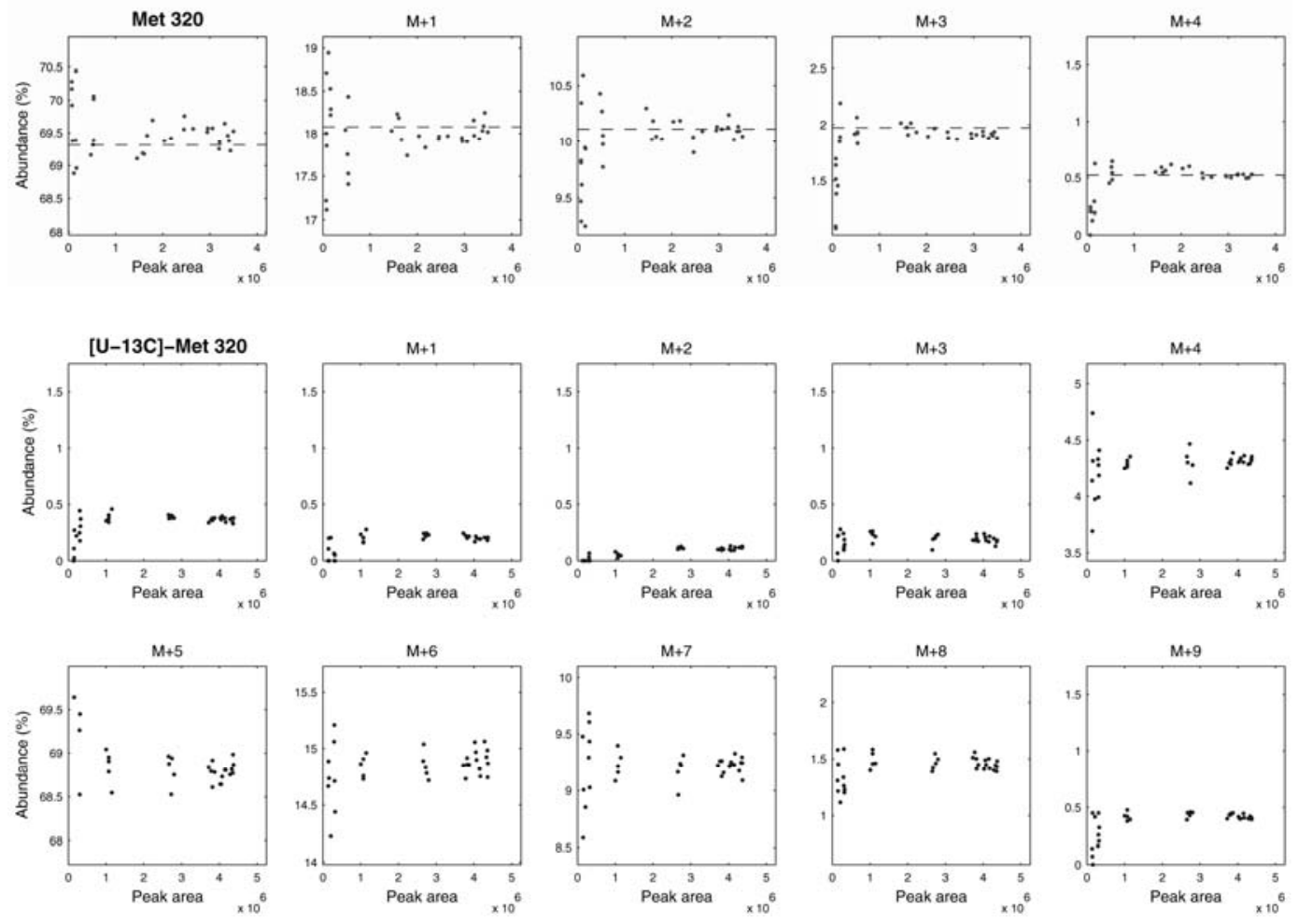

Met 320

\begin{tabular}{lrrr}
\hline Formula : & \multicolumn{3}{c}{$\mathrm{C}_{13} \mathrm{H}_{30} \mathrm{NO}_{2} \mathrm{Si}_{2} \mathrm{~S}$} \\
Exact mass : & \multicolumn{3}{c}{320.154} \\
C-atoms : & \multicolumn{2}{c}{$1-2-3-4-5$} \\
\hline $\mathrm{m} / \mathrm{z}$ & theory & data & difference \\
\hline 319 & 0.0 & 0.0 & 0.0 \\
\hline 320 & 100.0 & 100.0 & 0.0 \\
321 & 26.1 & 25.9 & -0.2 \\
322 & 14.6 & 14.6 & 0.0 \\
323 & 2.8 & 2.8 & 0.0 \\
324 & 0.8 & 0.8 & 0.0 \\
325 & 0.1 & 0.1 & 0.0 \\
326 & 0.0 & 0.0 & 0.0 \\
327 & 0.0 & 0.0 & 0.0 \\
328 & 0.0 & 0.1 & 0.1 \\
329 & 0.0 & 0.1 & 0.1 \\
330 & 0.0 & 0.0 & 0.0 \\
331 & 0.0 & 0.0 & 0.0 \\
\hline
\end{tabular}

\section{$\left[\mathrm{U}-{ }^{13} \mathrm{C}\right]-\mathrm{Met}$}

\begin{tabular}{rrrr}
\hline $\mathrm{m} / \mathrm{z}$ & theory & data & difference \\
\hline 319 & 0.0 & 1.4 & 1.4 \\
\hline 320 & 0.0 & 0.5 & 0.5 \\
321 & 0.0 & 0.3 & 0.3 \\
322 & 0.0 & 0.1 & 0.1 \\
323 & 0.0 & 0.3 & 0.3 \\
324 & 5.2 & 6.2 & 1.0 \\
325 & 100.0 & 100.0 & 0.0 \\
326 & 21.1 & 21.4 & 0.3 \\
327 & 13.3 & 13.4 & 0.1 \\
328 & 2.1 & 2.1 & 0.0 \\
329 & 0.6 & 0.6 & 0.0 \\
330 & 0.1 & 0.0 & -0.1 \\
331 & 0.0 & 0.0 & 0.0 \\
\hline
\end{tabular}

This fragment can be used for flux analysis. Mass isotopomer distributions should be obtained from the mass range $\mathrm{m} / \mathrm{z} 320-327$. 


\section{Phenylalanine (Phe)}
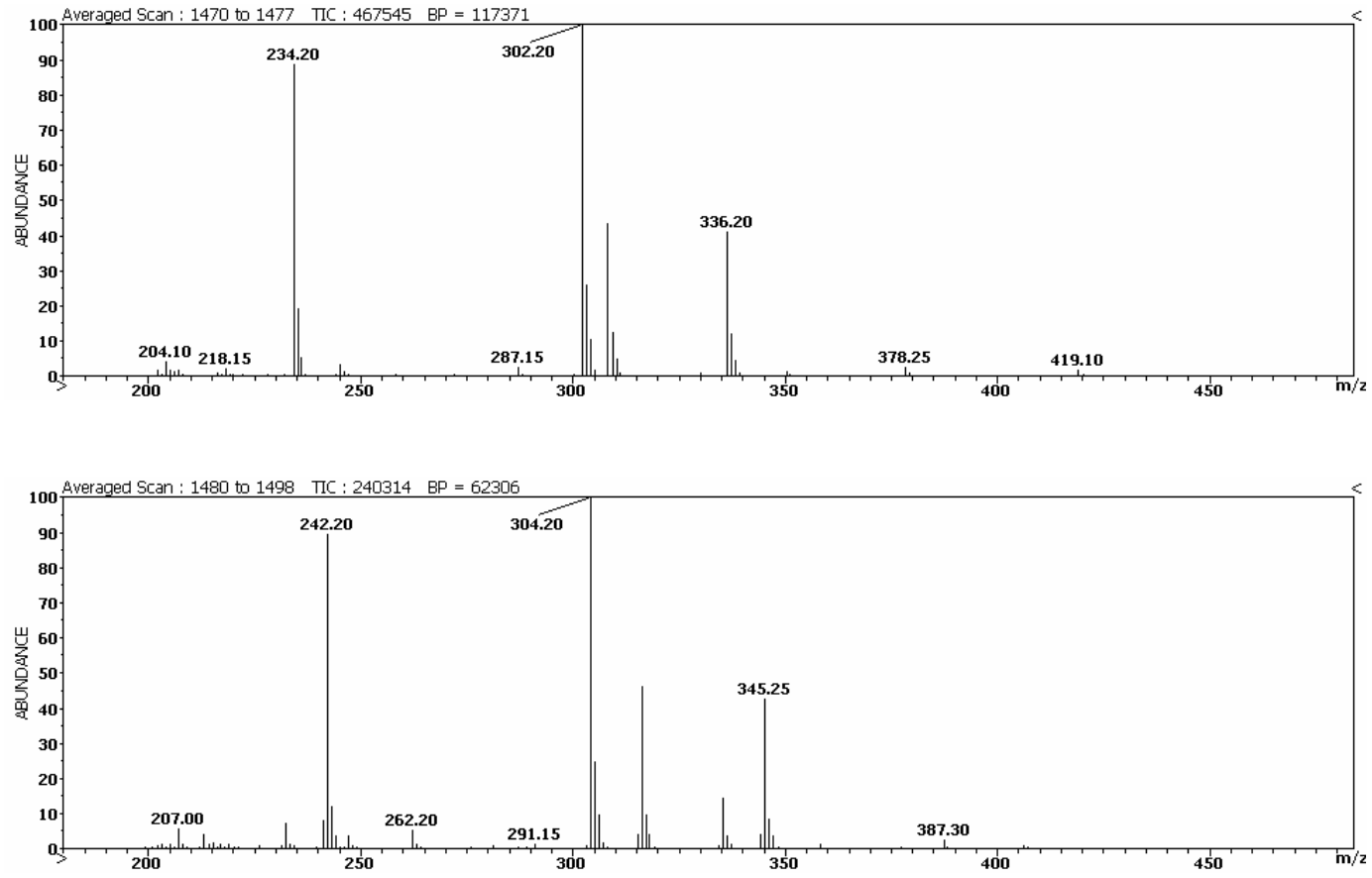<smiles>CC(C)(C)[Si](C)(C)NC(Cc1ccccc1)[Tl]Cl</smiles>

$$
\mathrm{C}_{21} \mathrm{H}_{39} \mathrm{NO}_{2} \mathrm{Si}_{2}
$$

Exact Mass: 393.2519 

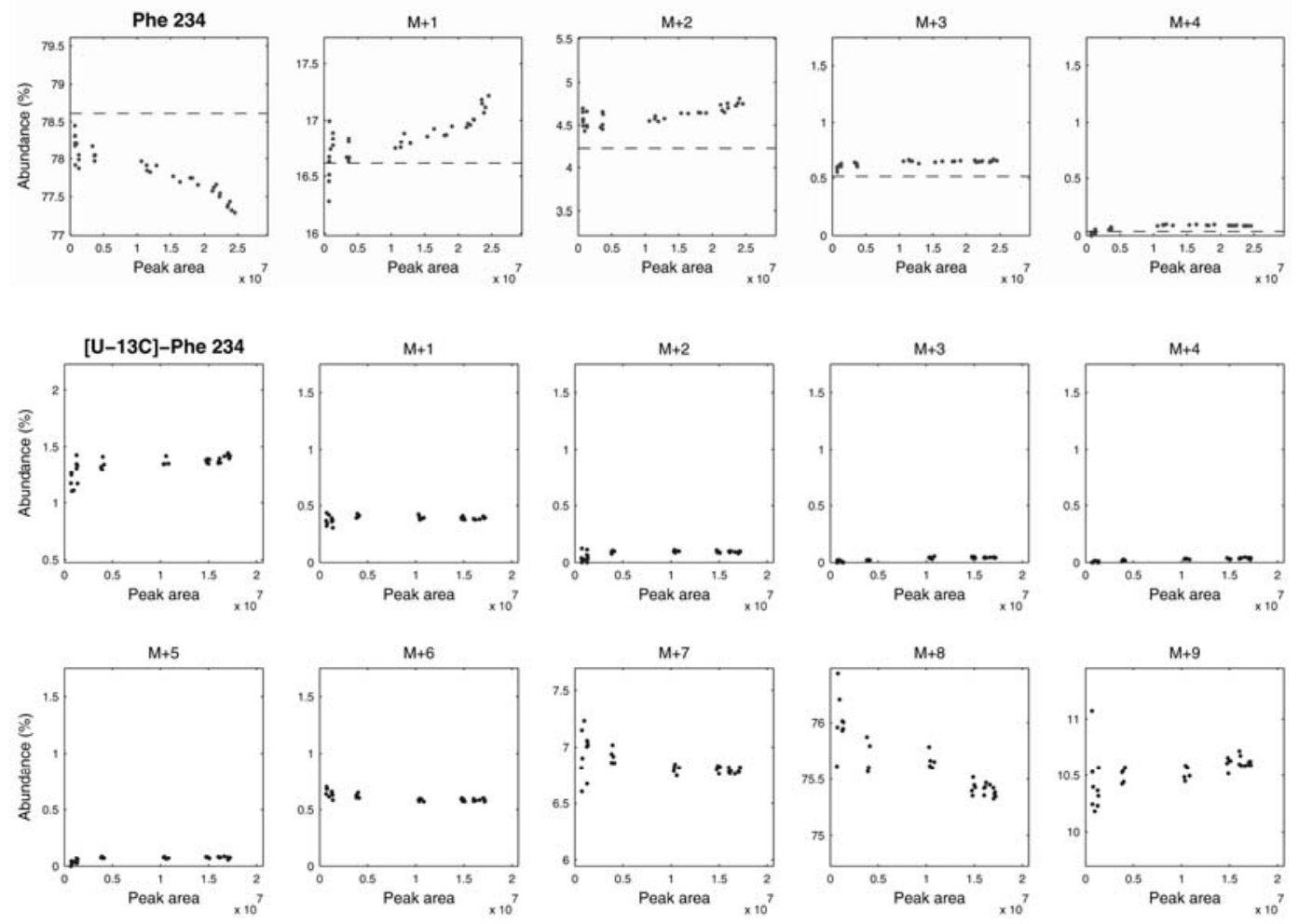

Phe 234

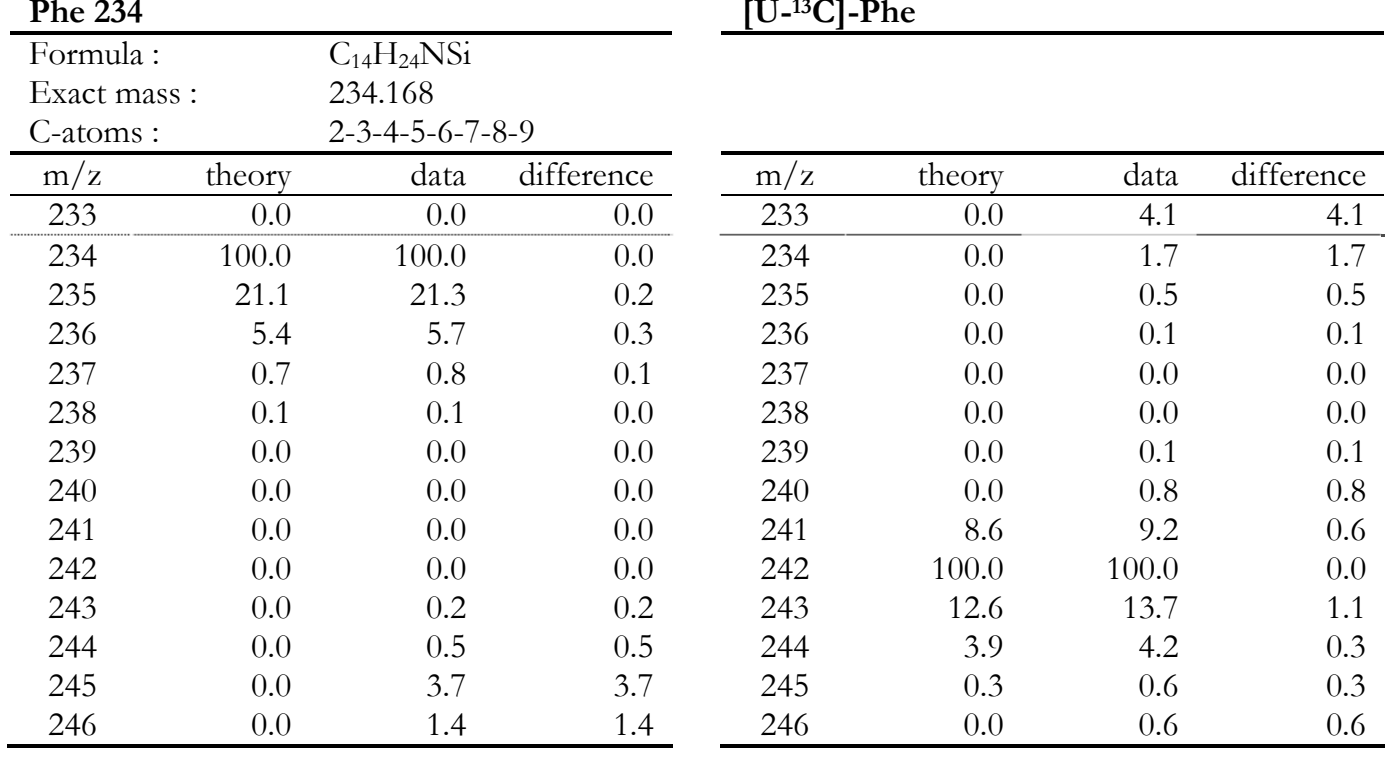

This fragment can be used for flux analysis. Mass isotopomer distributions should be obtained from the mass range $\mathrm{m} / \mathrm{z} 234-243$. 

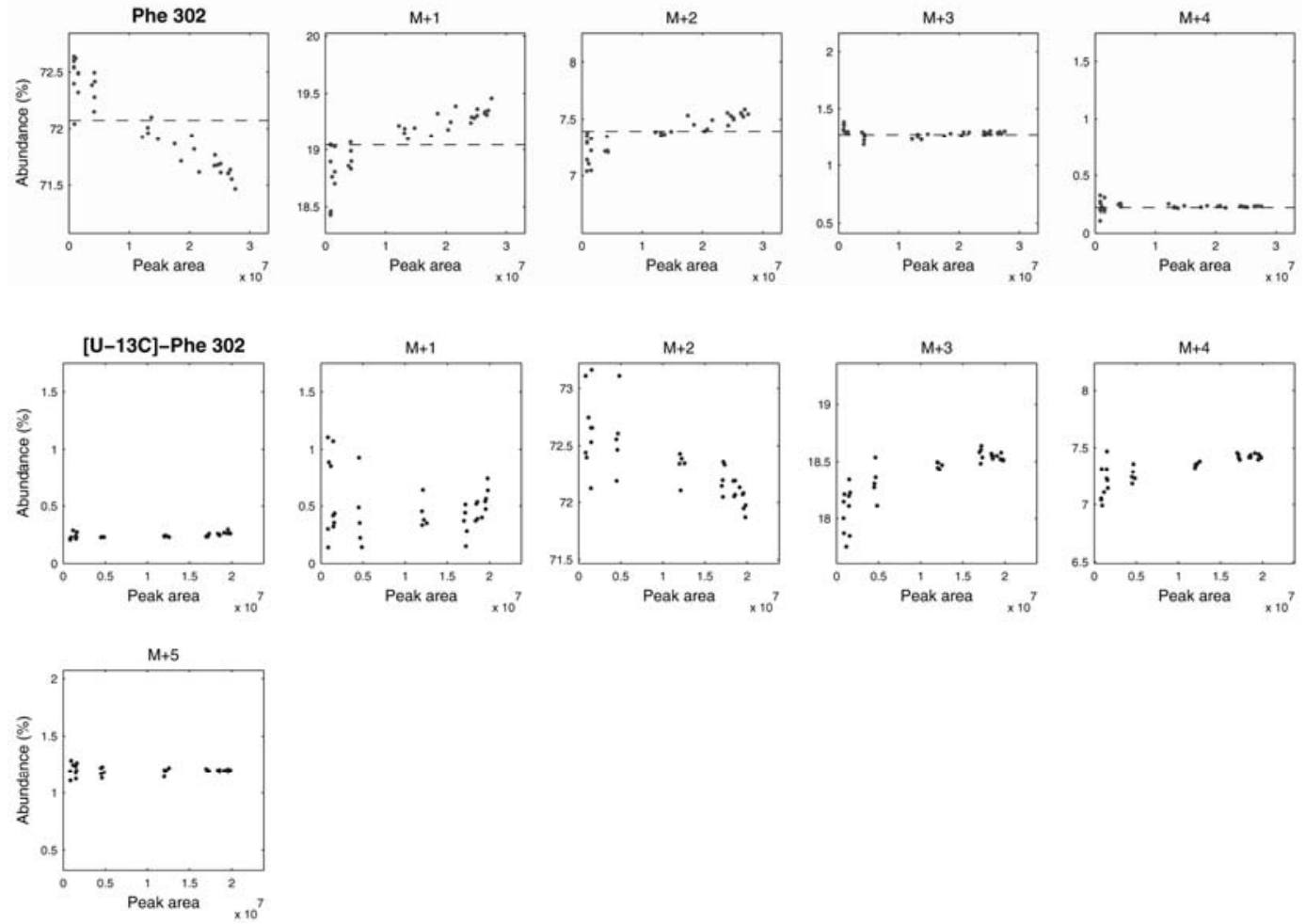

Phe 302

\begin{tabular}{lrrr}
\hline Formula : & \multicolumn{3}{l}{$\mathrm{C}_{14} \mathrm{H}_{32} \mathrm{O}_{2} \mathrm{NSi}_{2}$} \\
Exact mass : & \multicolumn{3}{l}{302.197} \\
C-atoms : & \multicolumn{3}{c}{$1-2$} \\
\hline $\mathrm{m} / \mathrm{z}$ & theory & data & difference \\
\hline 301 & 0.0 & 0.0 & 0.0 \\
\hline 302 & 100.0 & 100.0 & 0.0 \\
303 & 26.4 & 26.1 & -0.3 \\
304 & 10.3 & 9.9 & -0.4 \\
305 & 1.8 & 1.7 & -0.1 \\
306 & 0.3 & 0.3 & 0.0 \\
307 & 0.0 & 0.0 & 0.0 \\
\hline
\end{tabular}

$\left[\mathrm{U}-{ }^{13} \mathrm{C}\right]-\mathrm{Phe}$

This fragment can be used for flux analysis. 

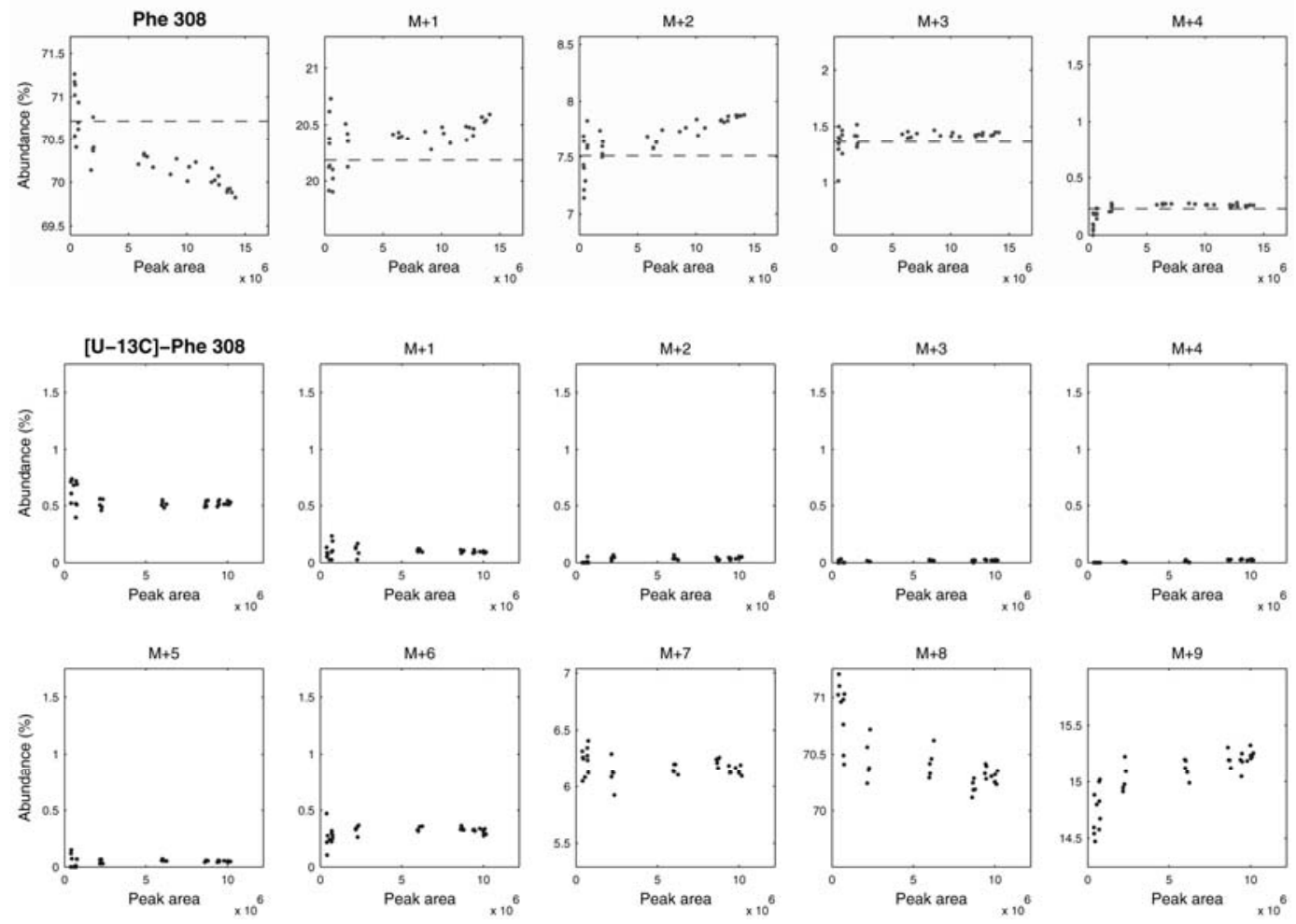

Phe 308

\begin{tabular}{lrrr}
\hline Formula : & \multicolumn{3}{c}{$\mathrm{C}_{16} \mathrm{H}_{30} \mathrm{ONSi}_{2}$} \\
Exact mass : & \multicolumn{3}{c}{308.187} \\
C-atoms : & \multicolumn{3}{c}{ 2-3-4-5-6-7-8-9 } \\
\hline $\mathrm{m} / \mathrm{z}$ & theory & data & difference \\
\hline 307 & 0.0 & 0.0 & 0.0 \\
\hline 308 & 100.0 & 100.0 & 0.0 \\
309 & 28.5 & 28.8 & 0.3 \\
310 & 10.6 & 10.6 & 0.0 \\
311 & 1.9 & 2.0 & 0.1 \\
312 & 0.3 & 0.4 & 0.1 \\
313 & 0.0 & 0.0 & 0.0 \\
314 & 0.0 & 0.0 & 0.0 \\
315 & 0.0 & 0.0 & 0.0 \\
316 & 0.0 & 0.0 & 0.0 \\
317 & 0.0 & 0.4 & 0.4 \\
318 & 0.0 & 0.3 & 0.3 \\
319 & 0.0 & 0.1 & 0.1 \\
320 & 0.0 & 0.2 & 0.2 \\
321 & 0.0 & 0.1 & 0.1 \\
\hline
\end{tabular}

\section{[U-133]-Phe}

\begin{tabular}{rrrr}
\hline $\mathrm{m} / \mathrm{z}$ & theory & data & difference \\
\hline 307 & 0.0 & 3.5 & 3.5 \\
\hline 308 & 0.0 & 0.8 & 0.8 \\
309 & 0.0 & 0.2 & 0.2 \\
310 & 0.0 & 0.1 & 0.1 \\
311 & 0.0 & 0.0 & 0.0 \\
312 & 0.0 & 0.0 & 0.0 \\
313 & 0.0 & 0.1 & 0.1 \\
314 & 0.0 & 0.4 & 0.4 \\
315 & 8.5 & 8.7 & 0.2 \\
316 & 100.0 & 100.0 & 0.0 \\
317 & 20.2 & 21.1 & 0.9 \\
318 & 8.5 & 8.8 & 0.3 \\
319 & 1.1 & 1.3 & 0.2 \\
320 & 0.2 & 0.2 & 0.0 \\
321 & 0.0 & 0.0 & 0.0 \\
\hline
\end{tabular}

This fragment can be used for flux analysis. Mass isotopomer distributions should be obtained from the mass range $\mathrm{m} / \mathrm{z} 308-316$. 

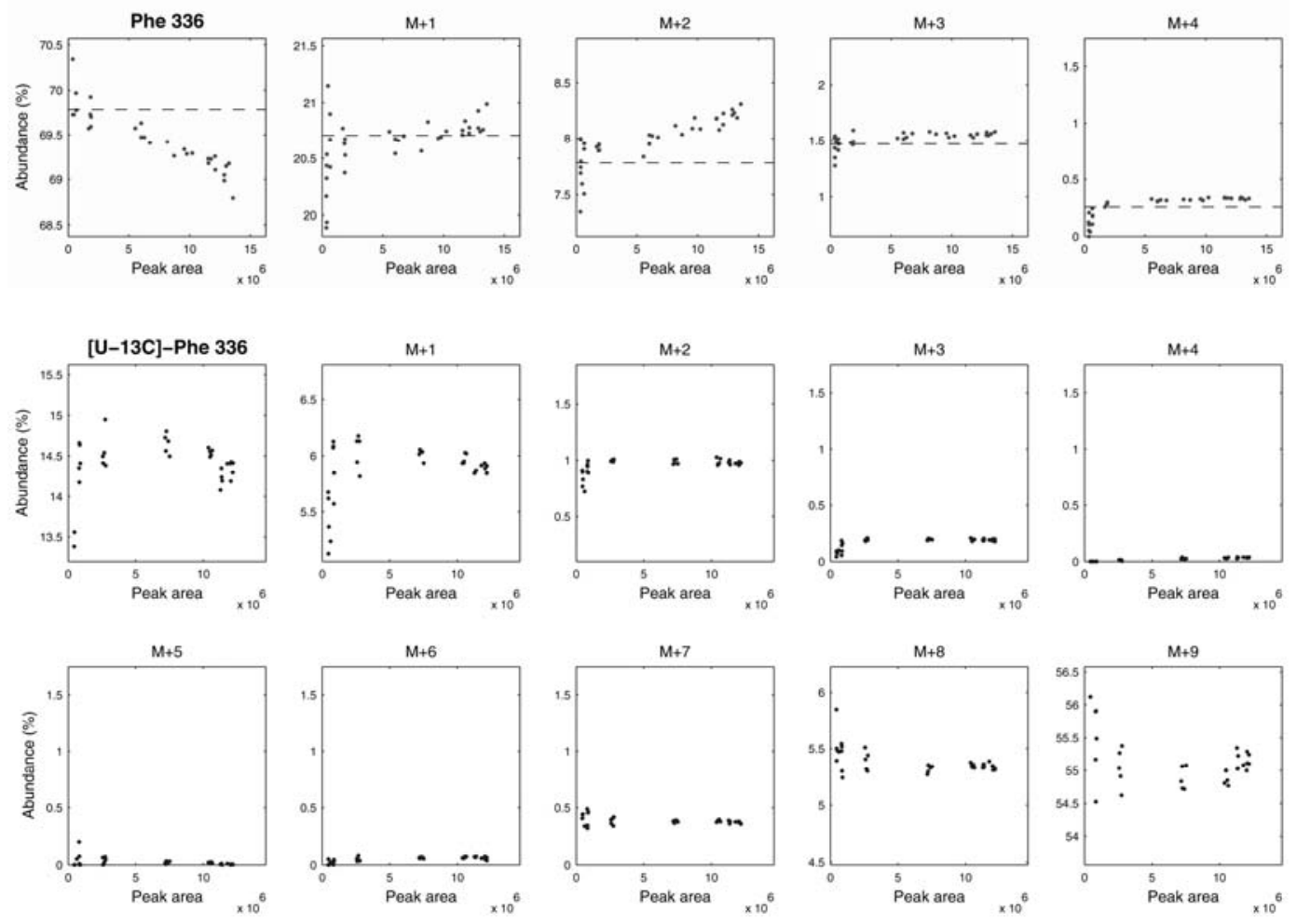

Phe 336

\begin{tabular}{lrrr}
\hline Formula : & \multicolumn{3}{c}{$\mathrm{C}_{17} \mathrm{H}_{30} \mathrm{O}_{2} \mathrm{NSi}_{2}$} \\
Exact mass : & \multicolumn{3}{c}{336.182} \\
C-atoms : & \multicolumn{3}{c}{ 1-2-3-4-5-6-7-8-9 } \\
\hline $\mathrm{m} / \mathrm{z}$ & theory & data & difference \\
\hline 335 & 0.0 & 0.0 & 0.0 \\
\hline 336 & 100.0 & 100.0 & 0.0 \\
337 & 29.7 & 29.4 & -0.3 \\
338 & 11.2 & 11.2 & 0.0 \\
339 & 2.1 & 2.2 & 0.1 \\
340 & 0.4 & 0.4 & 0.0 \\
341 & 0.0 & 0.1 & 0.1 \\
342 & 0.0 & 0.1 & 0.1 \\
343 & 0.0 & 0.0 & 0.0 \\
344 & 0.0 & 0.0 & 0.0 \\
345 & 0.0 & 0.1 & 0.1 \\
346 & 0.0 & 0.1 & 0.1 \\
347 & 0.0 & 0.1 & 0.1 \\
348 & 0.0 & 0.0 & 0.0 \\
349 & 0.0 & 0.4 & 0.4 \\
\hline
\end{tabular}

$\underline{\left[\mathrm{U}-{ }^{13} \mathrm{C}\right]-\mathrm{Phe}}$

\begin{tabular}{rrrr}
\hline $\mathrm{m} / \mathrm{z}$ & theory & data & difference \\
\hline 335 & 0.0 & 108.4 & 108.4 \\
\hline 336 & 0.0 & 26.4 & 26.4 \\
337 & 0.0 & 10.9 & 10.9 \\
338 & 0.0 & 1.8 & 1.8 \\
339 & 0.0 & 0.3 & 0.3 \\
340 & 0.0 & 0.0 & 0.0 \\
341 & 0.0 & 0.1 & 0.1 \\
342 & 0.0 & 0.1 & 0.1 \\
343 & 0.0 & 0.7 & 0.7 \\
344 & 9.7 & 9.8 & 0.1 \\
345 & 100.0 & 100.0 & 0.0 \\
346 & 20.3 & 20.8 & 0.5 \\
347 & 8.7 & 8.9 & 0.2 \\
348 & 1.1 & 1.3 & 0.2 \\
349 & 0.2 & 0.2 & 0.0 \\
\hline
\end{tabular}

This fragment can be used for flux analysis. Phenylalanine should not be $\mathrm{M}+9$ or $\mathrm{M}+8$ labeled for this fragment to be accurate (see the abundances at $\mathrm{m} / \mathrm{z} 336-338$ in $\left[\mathrm{U}-{ }^{13} \mathrm{C}\right]$ phenylalanine). 


\section{Aspartate (Asp)}
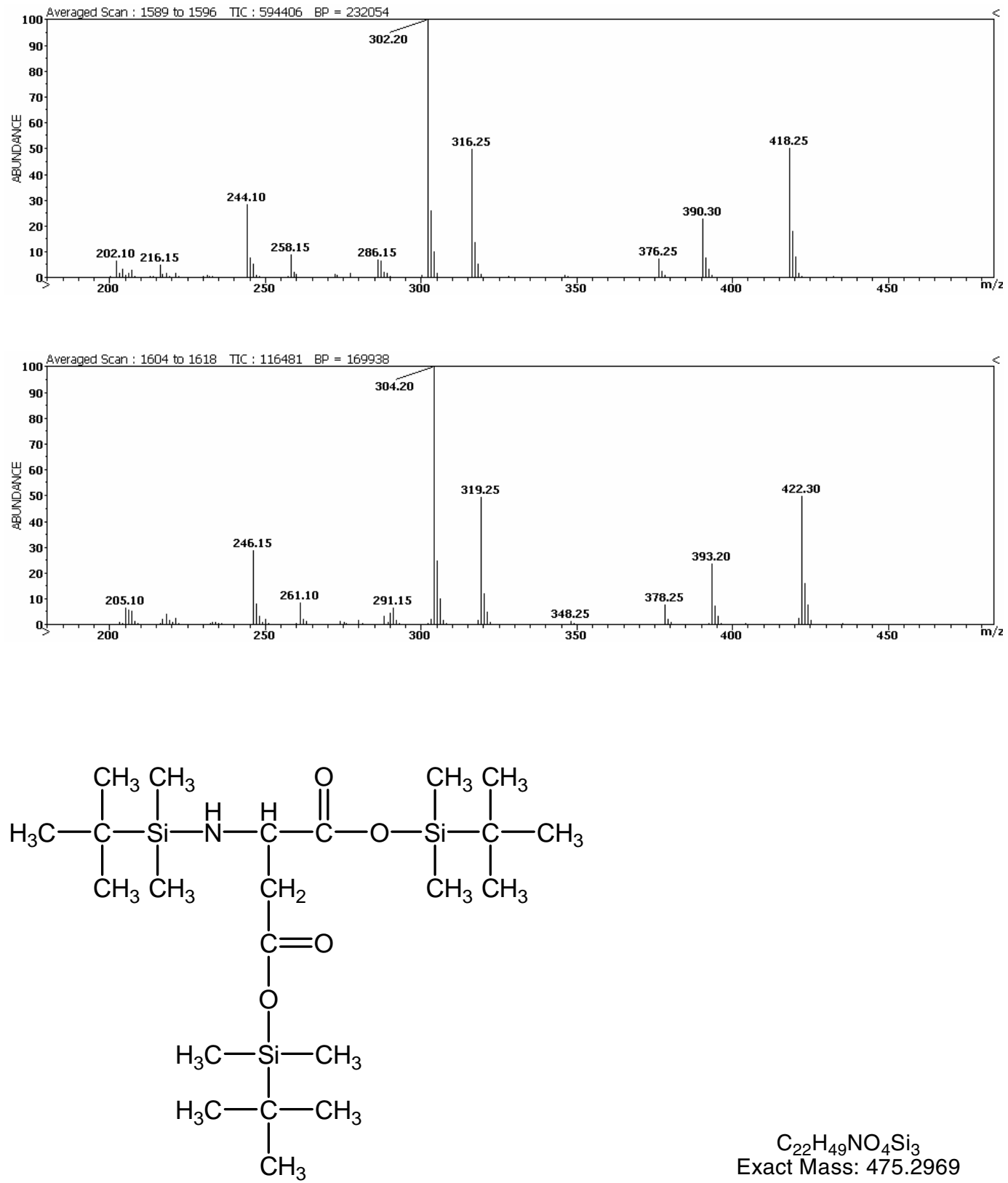

$$
\mathrm{C}_{22} \mathrm{H}_{49} \mathrm{NO}_{4} \mathrm{Si}_{3}
$$

Exact Mass: 475.2969 

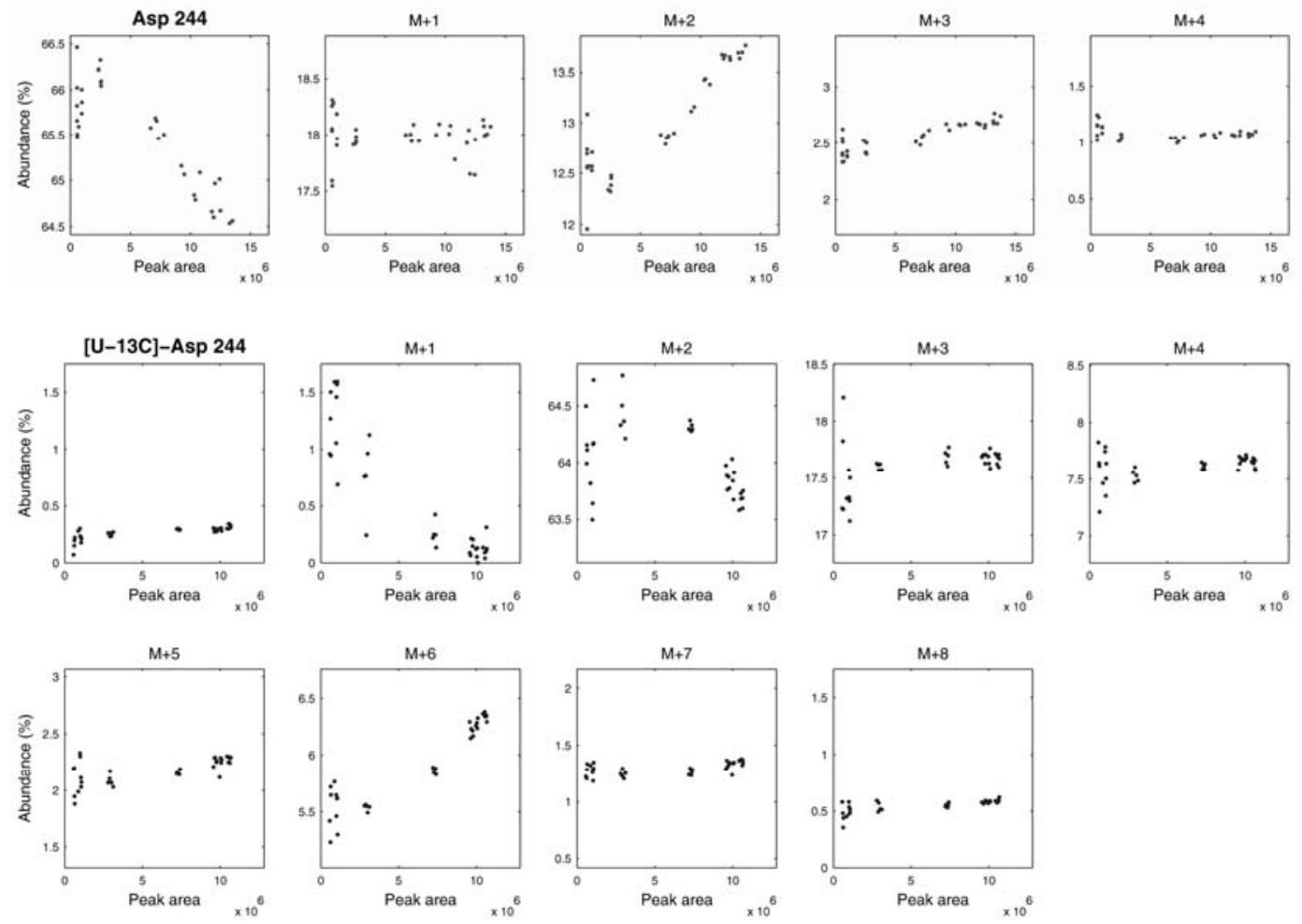

Asp 244

$\underline{\left[\mathrm{U}-{ }^{13} \mathrm{C}\right]-\mathrm{Asp}}$

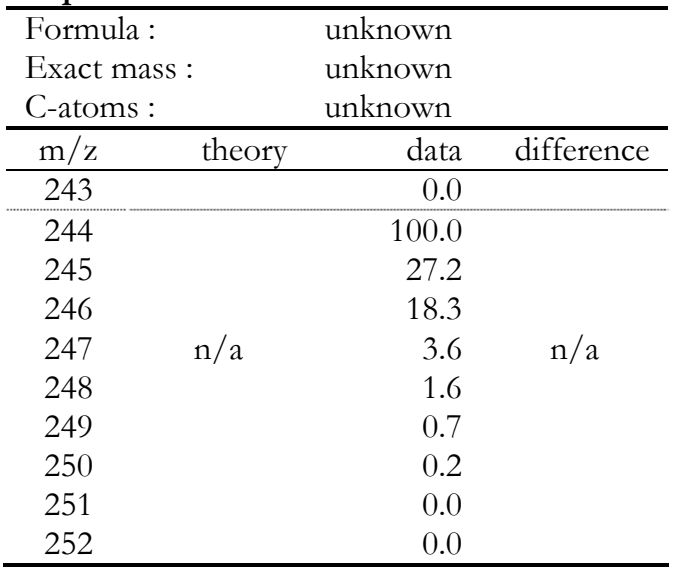

\begin{tabular}{ccrl}
\hline $\mathrm{m} / \mathrm{z}$ & theory & data & difference \\
\hline 243 & & 0.1 & \\
\hline 244 & & 0.3 & \\
245 & & 1.8 & \\
246 & & 100.0 & \\
247 & $\mathrm{n} / \mathrm{a}$ & 27.2 & $\mathrm{n} / \mathrm{a}$ \\
248 & & 11.7 & \\
249 & & 3.2 & \\
250 & & 8.2 & \\
251 & & 1.9 & \\
252 & & 0.8 & \\
\hline
\end{tabular}

This fragment should not be used for flux analysis. The relatively high $\mathrm{M}+2(18 \%$ of $\mathrm{M}+0)$ suggests that two fragments are overlapping. $\left[4-{ }^{13} \mathrm{C}\right]$-aspartate contains peaks at 244 and 247 , which further suggests the presence of two overlapping fragments. 

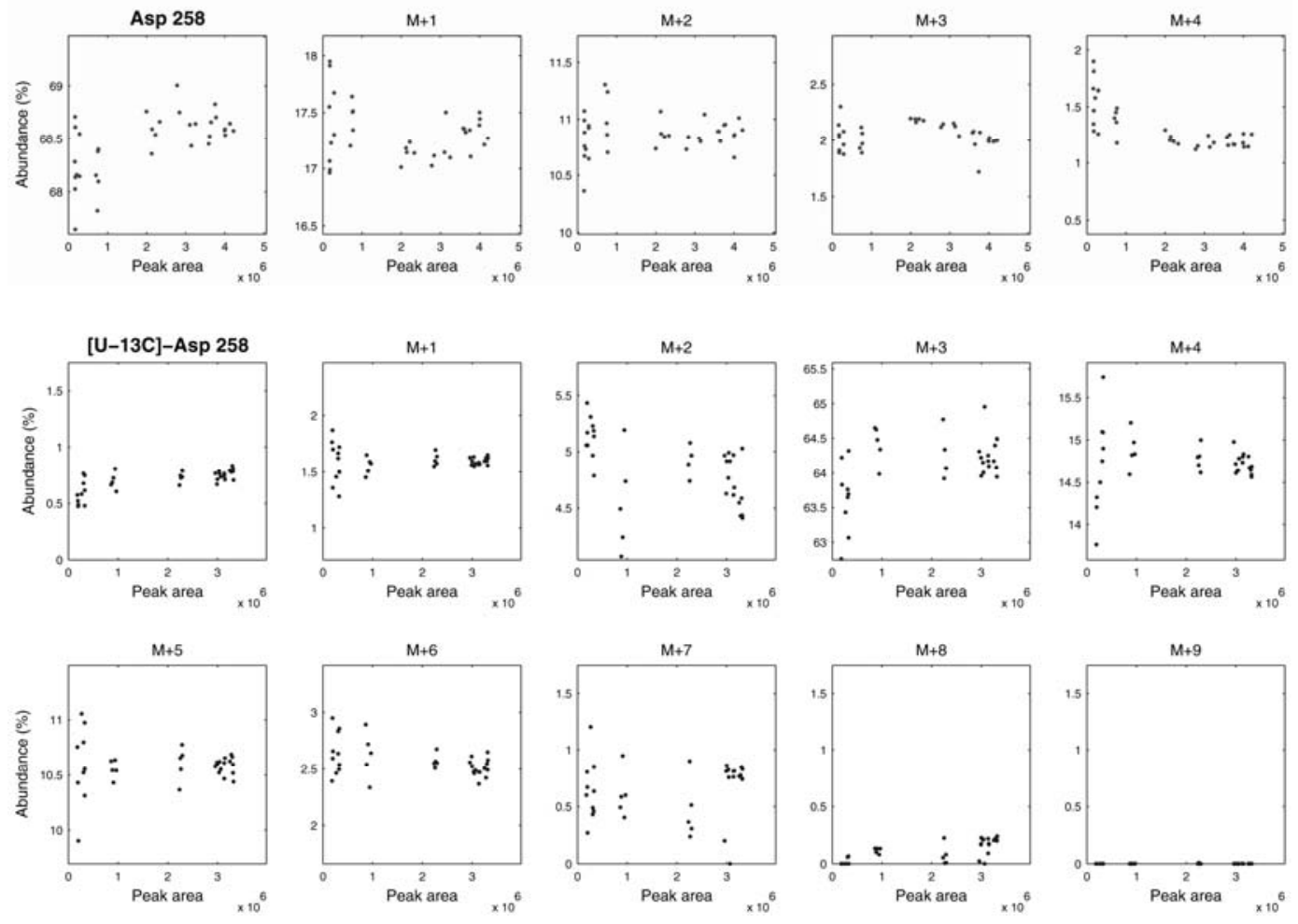

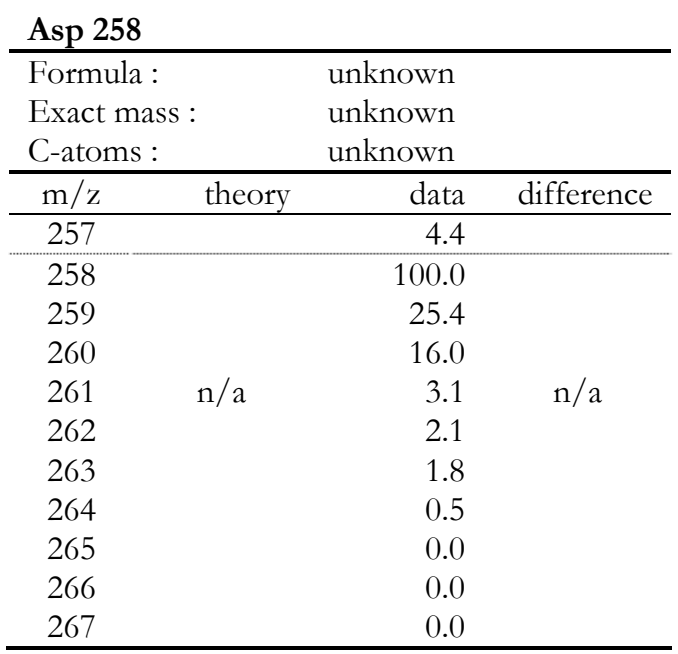

$\left[\mathrm{U}-{ }^{13} \mathrm{C}\right]-\mathrm{Asp}$

\begin{tabular}{ccrc}
\hline $\mathrm{m} / \mathrm{z}$ & theory & data & difference \\
\hline 257 & & 1.8 & \\
\hline 258 & & 1.0 & \\
259 & & 2.4 & \\
260 & & 7.6 & \\
261 & $\mathrm{n} / \mathrm{a}$ & 100.0 & $\mathrm{n} / \mathrm{a}$ \\
262 & & 23.2 & \\
263 & & 16.6 & \\
264 & & 4.2 & \\
265 & & 0.8 & \\
266 & & 0.1 & \\
267 & & 0.0 & \\
\hline
\end{tabular}

This fragment should not be used for flux analysis. M-1 is too high (4.4 mol\%) and the fragmentation is unknown. $\left[4-{ }^{13} \mathrm{C}\right]$-aspartate contains a peak at 259 but not at 258 , thus this fragment contains carbon 4 . 

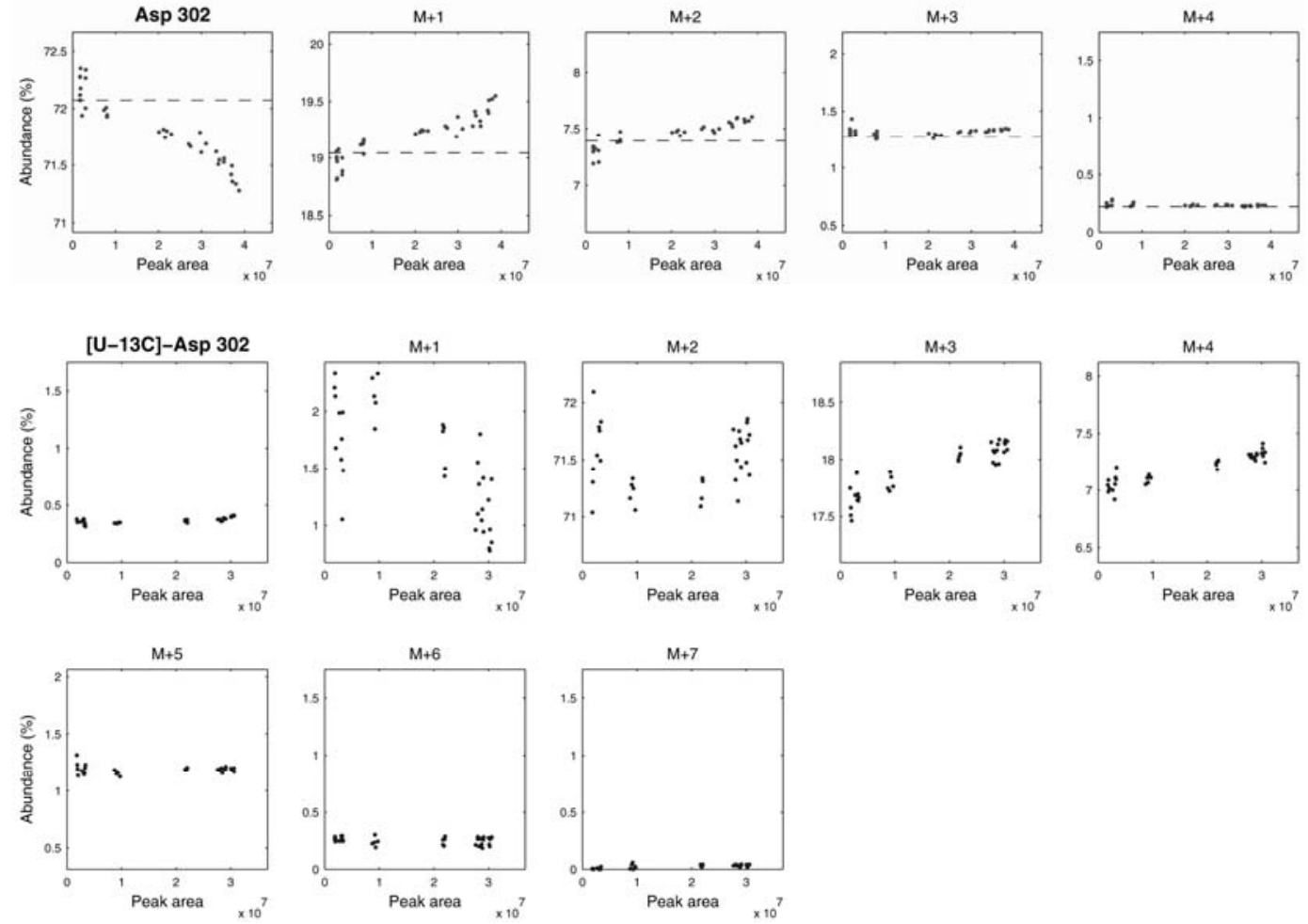

Asp 302

\begin{tabular}{lrrr}
\hline Formula : & \multicolumn{3}{l}{$\mathrm{C}_{14} \mathrm{H}_{32} \mathrm{O}_{2} \mathrm{NSi}_{2}$} \\
Exact mass : & \multicolumn{3}{c}{$1-2.197$} \\
C-atoms : & \multicolumn{3}{c}{} \\
\hline $\mathrm{m} / \mathrm{z}$ & theory & data & difference \\
\hline 301 & 0.0 & 0.0 & 0.0 \\
\hline 302 & 100.0 & 100.0 & 0.0 \\
303 & 26.4 & 26.4 & 0.0 \\
304 & 10.3 & 10.2 & -0.1 \\
305 & 1.8 & 1.8 & 0.0 \\
306 & 0.3 & 0.3 & 0.0 \\
307 & 0.0 & 0.0 & 0.0 \\
308 & 0.0 & 0.0 & 0.0 \\
309 & 0.0 & 0.0 & 0.0 \\
\hline
\end{tabular}

$\left[\mathrm{U}-{ }^{13} \mathrm{C}\right]-\mathrm{Asp}$

This fragment can be used for flux analysis. [4-13 C]-aspartate contains a peak at 302 but not at 303, thus this fragment does not contain carbon 4. 

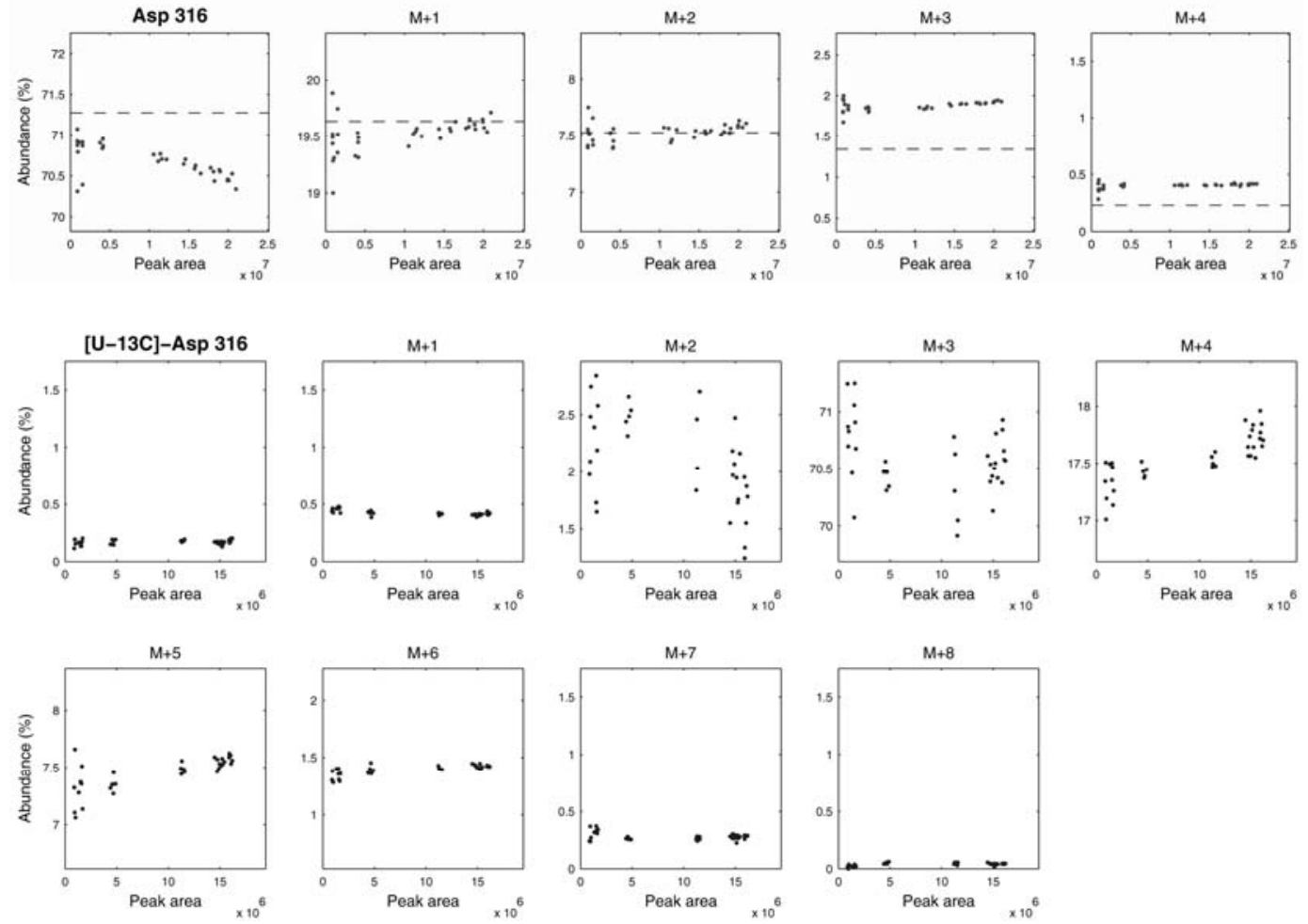

Asp 316

\begin{tabular}{lrrr}
\hline Formula : & \multicolumn{3}{c}{$\mathrm{C}_{15} \mathrm{H}_{34} \mathrm{NO}_{2} \mathrm{Si}_{2}$} \\
Exact mass : & \multicolumn{3}{c}{316.213} \\
C-atoms : & \multicolumn{3}{c}{$2-3-4$} \\
\hline $\mathrm{m} / \mathrm{z}$ & theory & data & difference \\
\hline 315 & 0.0 & 0.0 & 0.0 \\
\hline 316 & 100.0 & 100.0 & 0.0 \\
317 & 27.5 & 27.3 & -0.2 \\
318 & 10.6 & 10.5 & -0.1 \\
319 & 1.9 & 2.5 & 0.6 \\
320 & 0.3 & 0.6 & 0.3 \\
321 & 0.0 & 0.1 & 0.1 \\
322 & 0.0 & 0.0 & 0.0 \\
323 & 0.0 & 0.0 & 0.0 \\
324 & 0.0 & 0.0 & 0.0 \\
\hline
\end{tabular}

$\left[\mathrm{U}-{ }^{13} \mathrm{C}\right]-\mathrm{Asp}$

This fragment should not be used for flux analysis. It has a significant bias, i.e. $\mathrm{M}+0$ is too low $(-0.3$ $\mathrm{mol} \%)$ and $\mathrm{M}+3$ is too high $(+0.7 \mathrm{~mol} \%) \cdot\left[4-{ }^{13} \mathrm{C}\right]$-aspartate contains a peak at 317 but not at 316 , thus this fragment contains carbon 4. 

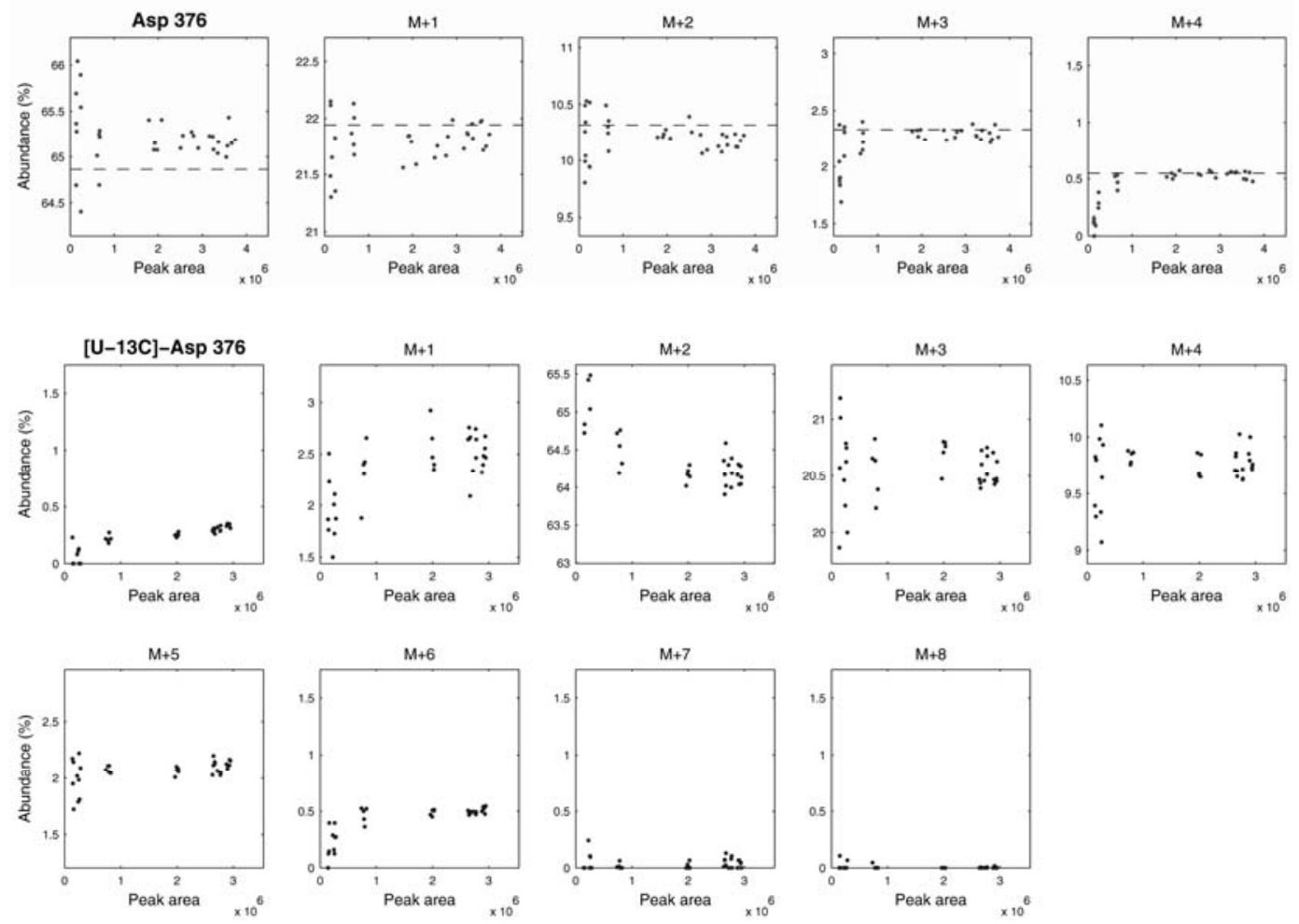

Asp 376

\begin{tabular}{lrrr}
\hline Formula : & \multicolumn{3}{c}{$\mathrm{C}_{16} \mathrm{H}_{38} \mathrm{O}_{3} \mathrm{NSi}_{3}$} \\
Exact mass : & \multicolumn{3}{c}{$1-2.216$} \\
C-atoms : & \multicolumn{3}{c}{} \\
\hline $\mathrm{m} / \mathrm{z}$ & theory & data & difference \\
\hline 375 & 0.0 & 0.1 & 0.1 \\
\hline 376 & 100.0 & 100.0 & 0.0 \\
377 & 33.8 & 33.5 & -0.3 \\
378 & 15.9 & 15.8 & -0.1 \\
379 & 3.6 & 3.5 & -0.1 \\
380 & 0.8 & 0.7 & -0.1 \\
381 & 0.1 & 0.0 & -0.1 \\
382 & 0.0 & 0.0 & 0.0 \\
383 & 0.0 & 0.1 & 0.1 \\
384 & 0.0 & 0.0 & 0.0 \\
\hline
\end{tabular}

$\left[\mathrm{U}-{ }^{13} \mathrm{C}\right]-\mathrm{Asp}$

This fragment can be used for flux analysis. Note that this fragment is noisy at low concentrations, and thus should be measured at higher concentrations only. $\left[4-{ }^{13} \mathrm{C}\right]$-aspartate contains a peak at 376 but not at 377, thus this fragment does not contain carbon 4 . 

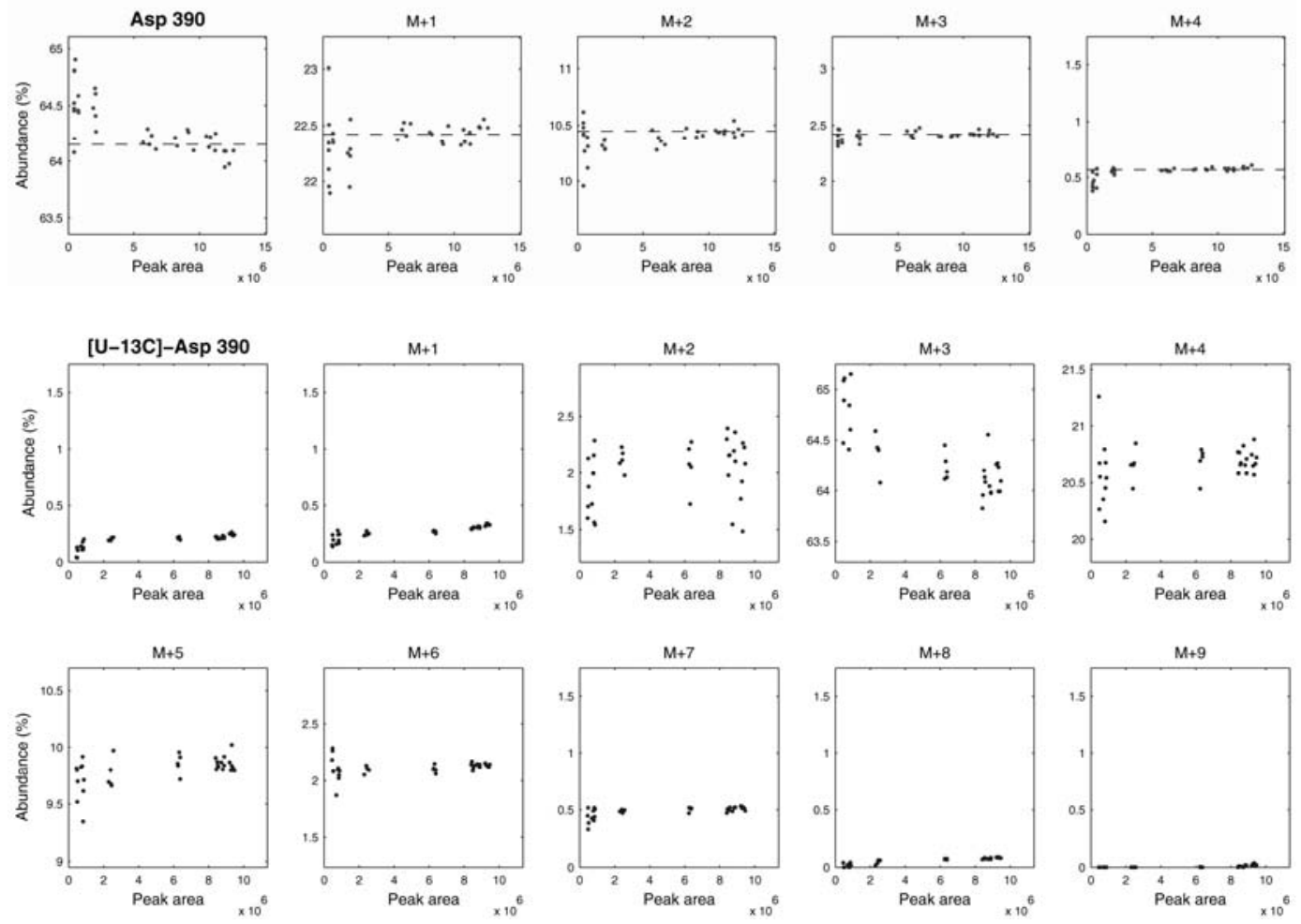

Asp 390

\begin{tabular}{lrrr}
\hline Formula : & \multicolumn{3}{c}{$\mathrm{C}_{17} \mathrm{H}_{40} \mathrm{O}_{3} \mathrm{NSi}_{3}$} \\
Exact mass : & \multicolumn{3}{c}{390.232} \\
C-atoms : & \multicolumn{3}{c}{$2-3-4$} \\
\hline $\mathrm{m} / \mathrm{z}$ & theory & data & difference \\
\hline 389 & 0.0 & 0.0 & 0.0 \\
\hline 390 & 100.0 & 100.0 & 0.0 \\
391 & 35.0 & 34.8 & -0.2 \\
392 & 16.3 & 16.1 & -0.2 \\
393 & 3.8 & 3.7 & -0.1 \\
394 & 0.9 & 0.8 & -0.1 \\
395 & 0.1 & 0.0 & -0.1 \\
396 & 0.0 & 0.0 & 0.0 \\
397 & 0.0 & 0.0 & 0.0 \\
398 & 0.0 & 0.0 & 0.0 \\
399 & 0.0 & 0.0 & 0.0 \\
\hline
\end{tabular}

\section{$\left[\mathrm{U}_{-13}{ }^{13} \mathrm{C}\right]-\mathrm{Asp}$}

\begin{tabular}{rrrr}
\hline $\mathrm{m} / \mathrm{z}$ & theory & data & difference \\
\hline 389 & 0.0 & 0.0 & 0.0 \\
\hline 390 & 0.0 & 0.2 & 0.2 \\
391 & 0.0 & 0.3 & 0.3 \\
392 & 3.1 & 3.1 & 0.0 \\
393 & 100.0 & 100.0 & 0.0 \\
394 & 31.8 & 31.8 & 0.0 \\
395 & 15.2 & 15.0 & -0.2 \\
396 & 3.3 & 3.2 & -0.1 \\
397 & 0.8 & 0.7 & -0.1 \\
398 & 0.1 & 0.0 & -0.1 \\
399 & 0.0 & 0.0 & 0.0 \\
\hline
\end{tabular}

This fragment can be used for flux analysis. Note that this fragment is noisy at low concentrations, and thus should be measured at higher concentrations only. $\left[4-{ }^{13} \mathrm{C}\right]$-aspartate contains a peak at 391 but not at 390, thus this fragment contains carbon 4. 

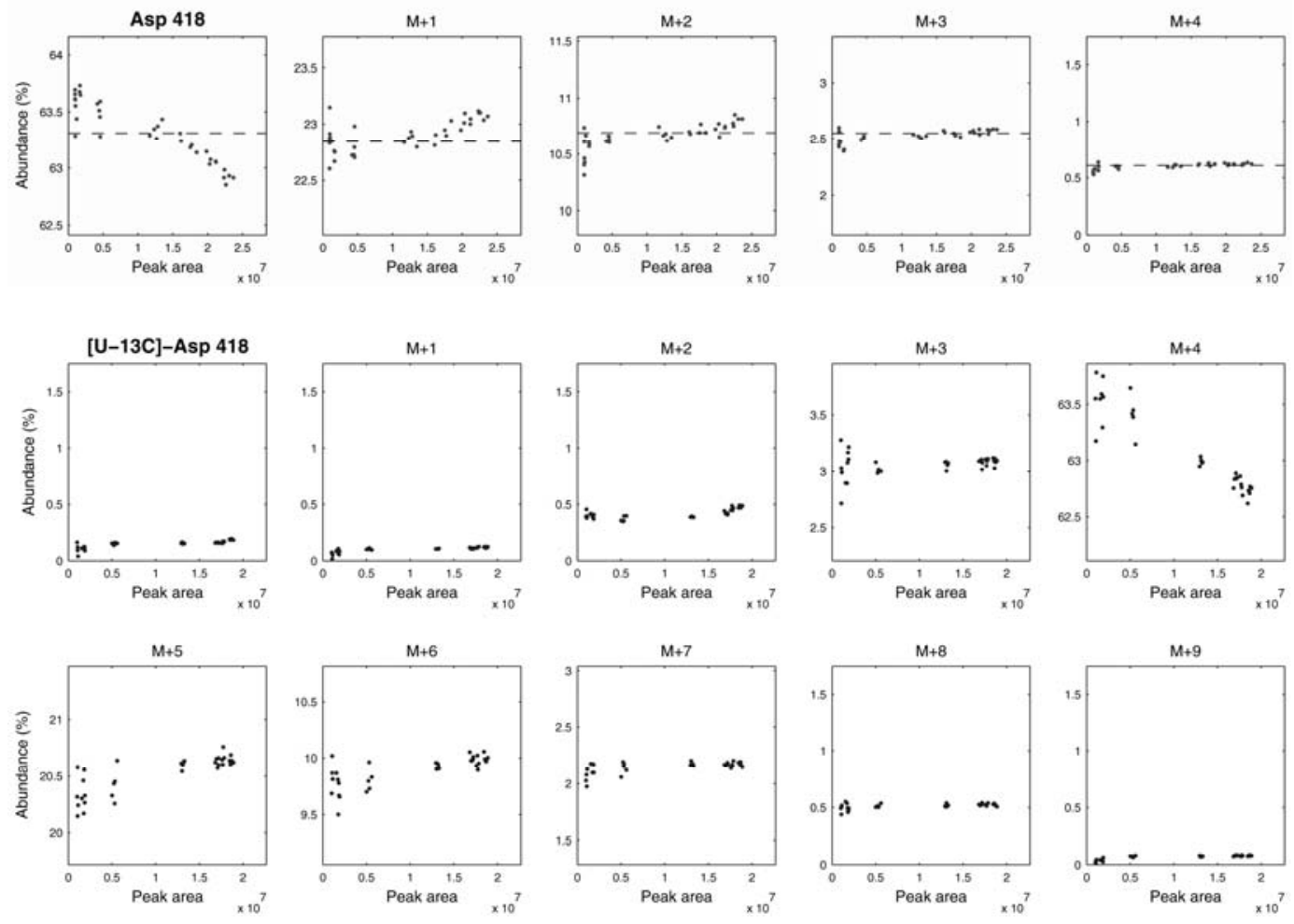

Asp 418

\begin{tabular}{lrrr}
\hline Formula : & \multicolumn{3}{c}{$\mathrm{C}_{18} \mathrm{H}_{40} \mathrm{O}_{4} \mathrm{NSi}_{3}$} \\
Exact mass : & \multicolumn{3}{c}{418.227} \\
C-atoms : & \multicolumn{3}{c}{$1-2-3-4$} \\
\hline $\mathrm{m} / \mathrm{z}$ & theory & data & difference \\
\hline 417 & 0.0 & 0.0 & 0.0 \\
\hline 418 & 100.0 & 100.0 & 0.0 \\
419 & 36.1 & 35.8 & -0.3 \\
420 & 16.9 & 16.7 & -0.2 \\
421 & 4.0 & 3.9 & -0.1 \\
422 & 1.0 & 0.9 & -0.1 \\
423 & 0.2 & 0.1 & -0.1 \\
424 & 0.0 & 0.0 & 0.0 \\
425 & 0.0 & 0.0 & 0.0 \\
426 & 0.0 & 0.0 & 0.0 \\
427 & 0.0 & 0.0 & 0.0 \\
428 & 0.0 & 0.0 & 0.0 \\
\hline
\end{tabular}

$\left[\mathrm{U}-{ }^{13} \mathrm{C}\right]-\mathrm{Asp}$

\begin{tabular}{rrrr}
\hline $\mathrm{m} / \mathrm{z}$ & theory & data & difference \\
\hline 417 & 0.0 & 0.0 & 0.0 \\
\hline 418 & 0.0 & 0.2 & 0.2 \\
419 & 0.0 & 0.1 & 0.1 \\
420 & 0.0 & 0.6 & 0.6 \\
421 & 4.1 & 4.7 & 0.6 \\
422 & 100.0 & 100.0 & 0.0 \\
423 & 31.9 & 31.9 & 0.0 \\
424 & 15.4 & 15.3 & -0.1 \\
425 & 3.3 & 3.3 & 0.0 \\
426 & 0.8 & 0.8 & 0.0 \\
427 & 0.1 & 0.1 & 0.0 \\
428 & 0.0 & 0.0 & 0.0 \\
\hline
\end{tabular}

This fragment can be used for flux analysis. $\left[4-{ }^{13} \mathrm{C}\right]$-aspartate contains a peak at 419 but not at 418 , thus this fragment contains carbon 4. 


\section{Glutamate (Glu)}
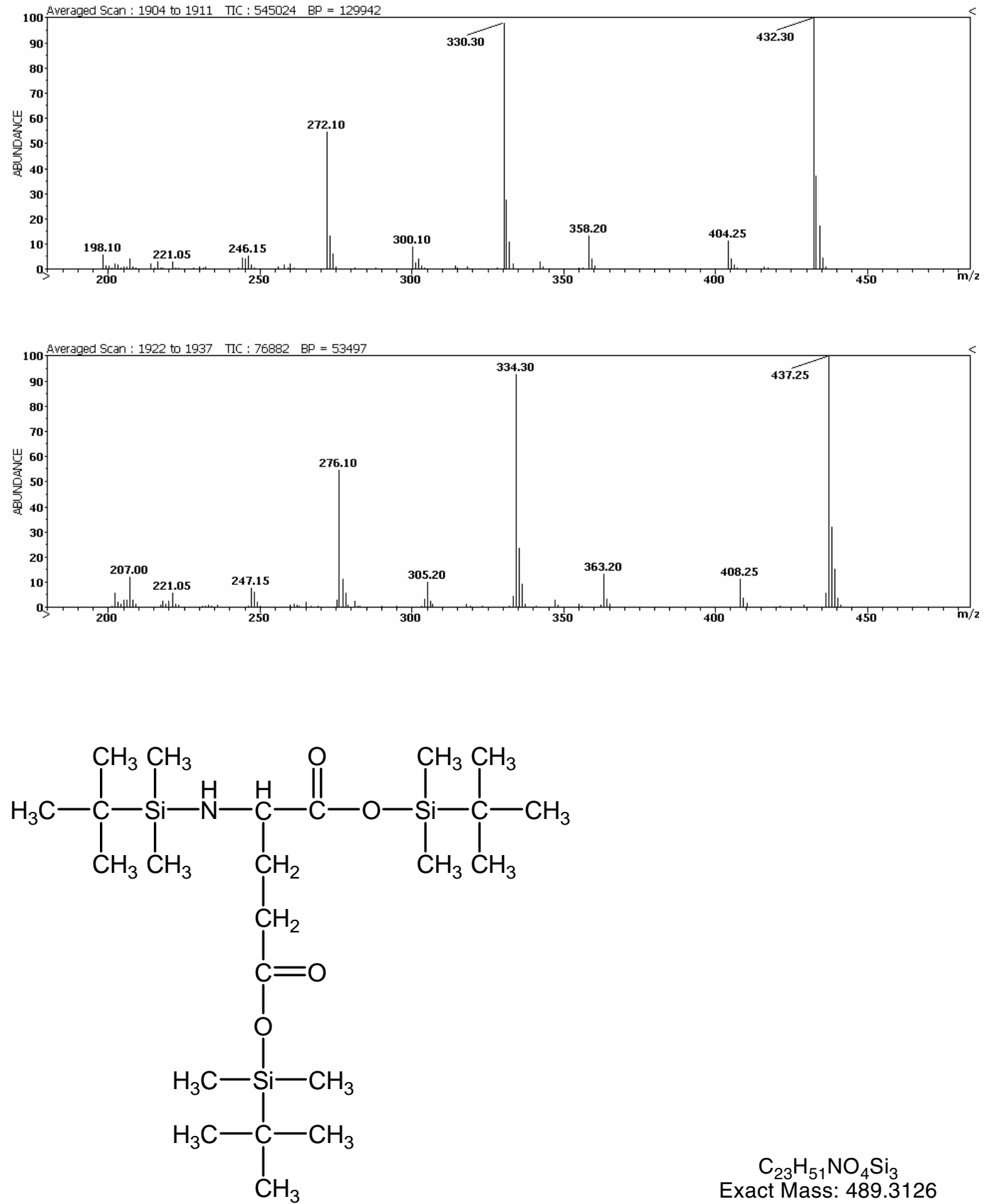

$\mathrm{C}_{23} \mathrm{H}_{51} \mathrm{NO}_{4} \mathrm{Si}_{3}$

Exact Mass: 489.3126 

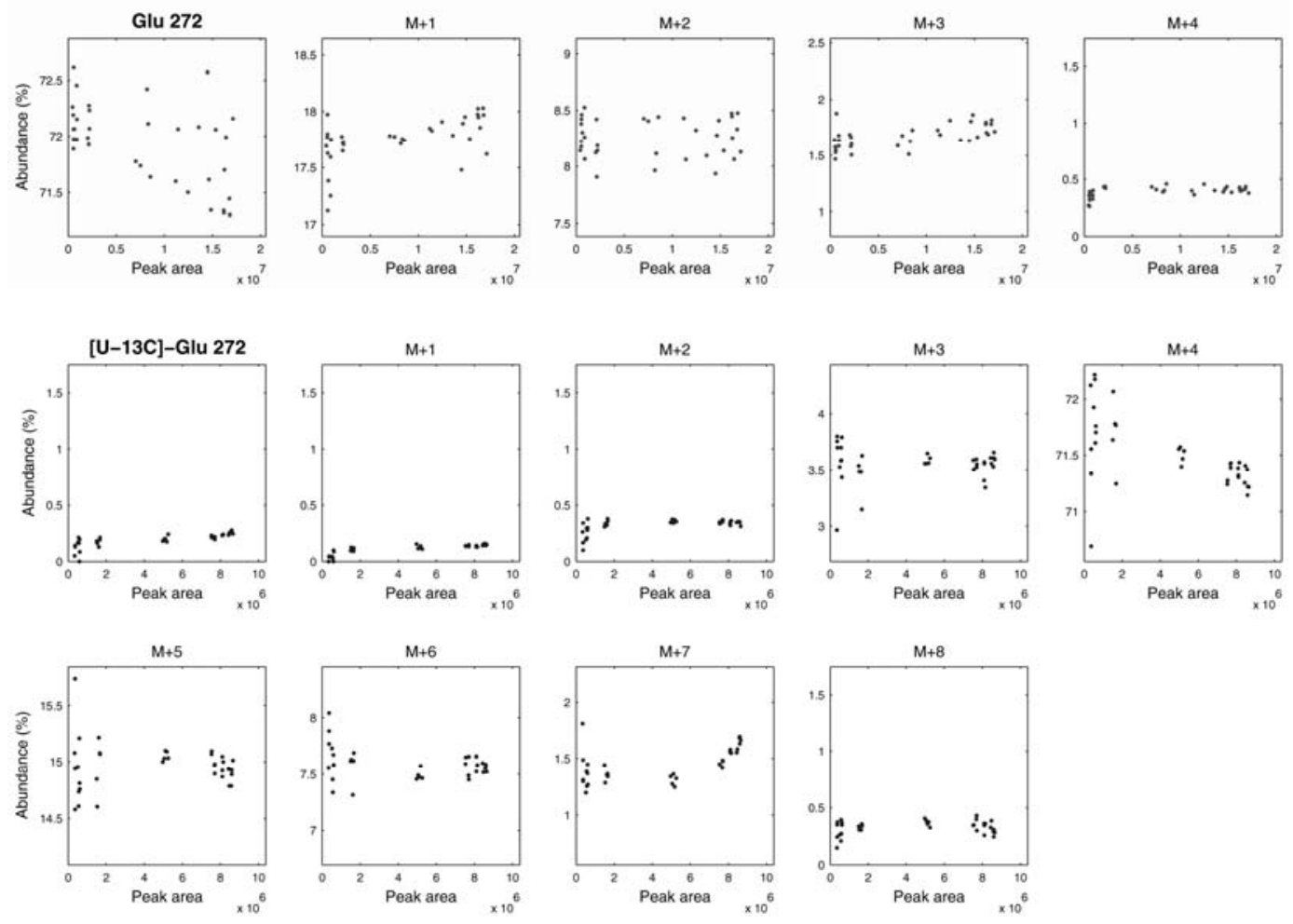

Glu 272

$\left[\mathrm{U}-{ }^{13} \mathrm{C}\right]-\mathrm{Glu}$

\begin{tabular}{|c|c|c|c|}
\hline \multicolumn{2}{|c|}{$\begin{array}{l}\text { Formula : } \\
\text { Exact mass : } \\
\text { C-atoms : }\end{array}$} & \multicolumn{2}{|l|}{$\begin{array}{l}\text { unknown } \\
\text { unknown } \\
\text { unknown }\end{array}$} \\
\hline $\mathrm{m} / \mathrm{z}$ & theory & data & difference \\
\hline 271 & & 0.0 & \\
\hline 272 & & 100.0 & \\
\hline 273 & & 24.4 & \\
\hline 274 & & 11.4 & \\
\hline 275 & $\mathrm{n} / \mathrm{a}$ & 2.2 & $\mathrm{n} / \mathrm{a}$ \\
\hline 276 & & 0.6 & \\
\hline 277 & & 0.1 & \\
\hline 278 & & 0.0 & \\
\hline 279 & & 0.1 & \\
\hline 280 & & 0.0 & \\
\hline
\end{tabular}

\begin{tabular}{crrr}
\hline $\mathrm{m} / \mathrm{z}$ & theory & data & difference \\
\hline 271 & & 0.0 & \\
\hline 272 & & 0.2 & \\
273 & & 0.1 & \\
274 & & 0.4 & \\
275 & $\mathrm{n} / \mathrm{a}$ & 4.9 & $\mathrm{n} / \mathrm{a}$ \\
276 & & 100.0 & \\
277 & & 20.8 & \\
278 & & 10.5 & \\
279 & & 1.7 & \\
280 & & 0.5 & \\
\hline
\end{tabular}

This fragment cannot be used for flux analysis. The fragmentation is unknown. $\left[5-{ }^{13} \mathrm{C}\right]$-glutamine contains a peak at 273 but not at 272, thus this fragment contains carbon 5 . 

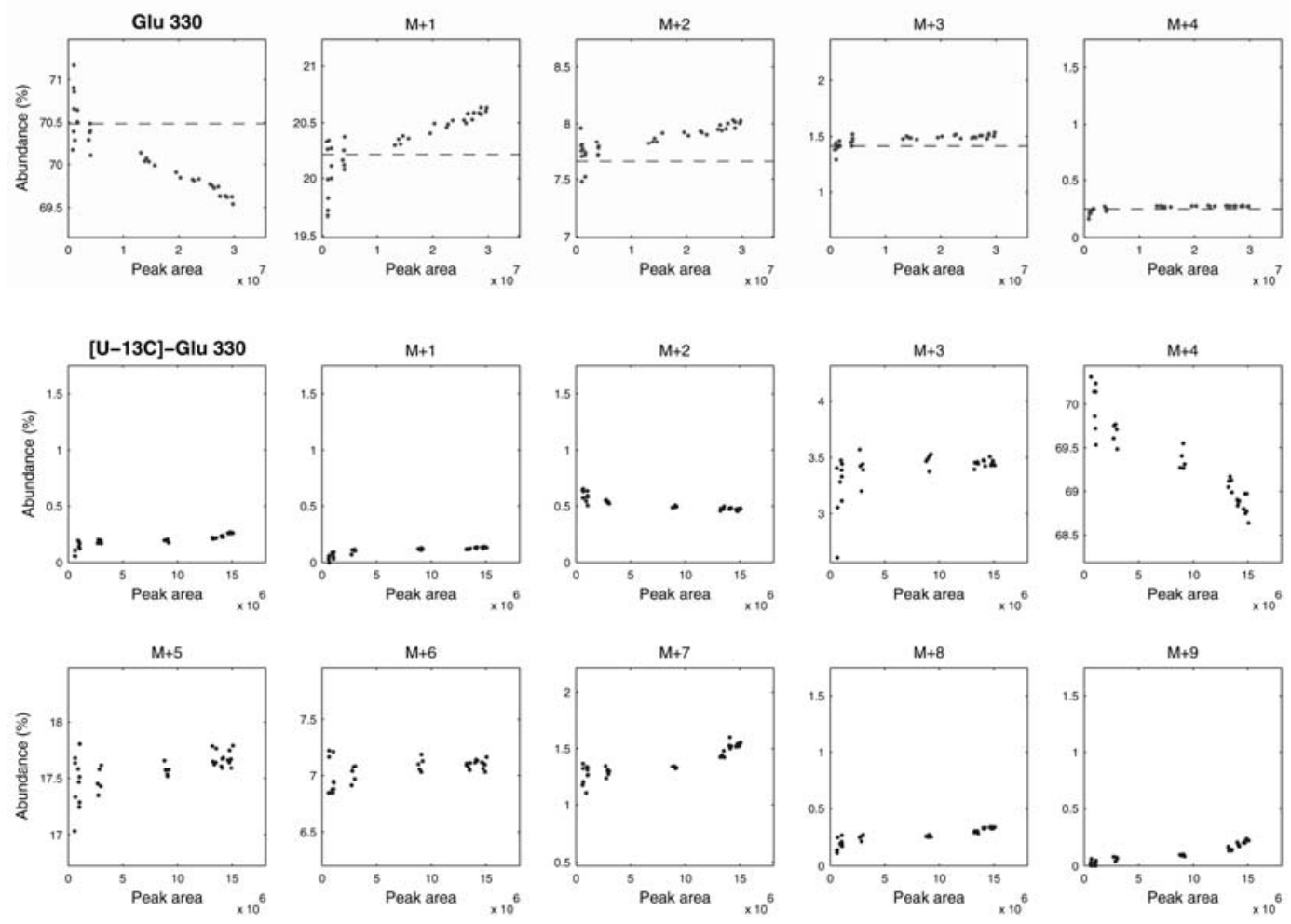

Glu 330

\begin{tabular}{|c|c|c|c|}
\hline \multicolumn{2}{|c|}{$\begin{array}{l}\text { Formula : } \\
\text { Exact mass : } \\
\text { C-atoms : }\end{array}$} & \multicolumn{2}{|c|}{$\begin{array}{l}\mathrm{C}_{16} \mathrm{H}_{36} \mathrm{O}_{2} \mathrm{NSi}_{2} \\
330.228 \\
2-3-4-5\end{array}$} \\
\hline $\mathrm{m} / \mathrm{z}$ & theory & data & difference \\
\hline 329 & 0.0 & 0.0 & 0.0 \\
\hline 330 & 100.0 & 100.0 & 0.0 \\
\hline 331 & 28.7 & 28.5 & -0.2 \\
\hline 332 & 10.9 & 10.9 & 0.0 \\
\hline 333 & 2.0 & 2.1 & 0.1 \\
\hline 334 & 0.3 & 0.3 & 0.0 \\
\hline 335 & 0.0 & 0.0 & 0.0 \\
\hline 336 & 0.0 & 0.0 & 0.0 \\
\hline 337 & 0.0 & 0.4 & 0.4 \\
\hline 338 & 0.0 & 0.1 & 0.1 \\
\hline 339 & 0.0 & 0.1 & 0.1 \\
\hline
\end{tabular}

$\left[\mathrm{U}-{ }^{13} \mathrm{C}\right]-\mathrm{Glu}$

This fragment can be used for flux analysis. Mass isotopomer distributions should be obtained from the mass range $\mathrm{m} / \mathrm{z} 330-336 \cdot\left[5^{-13} \mathrm{C}\right]$-glutamine contains a peak at 331 but not at 330 , thus this fragment contains carbon 5 . 

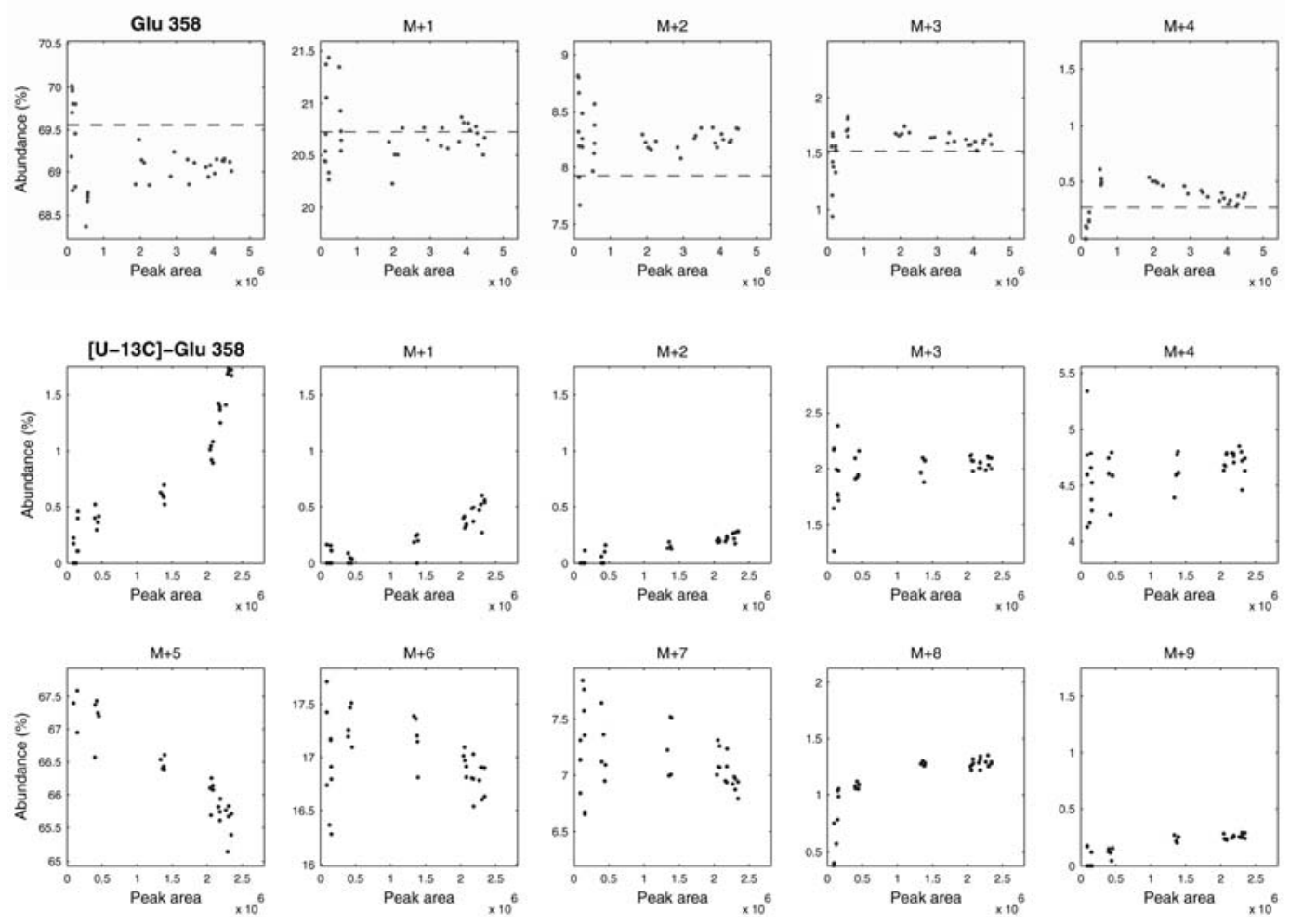

\section{Glu 358}

\begin{tabular}{lrrr}
\hline Formula : & \multicolumn{3}{c}{$\mathrm{C}_{17} \mathrm{H}_{36} \mathrm{NO}_{3} \mathrm{Si}_{2}$} \\
Exact mass : & \multicolumn{3}{c}{358.223} \\
C-atoms : & \multicolumn{3}{c}{$1-2-3-4-5$} \\
\hline $\mathrm{m} / \mathrm{z}$ & theory & data & difference \\
\hline 357 & 0.0 & 1.8 & 1.8 \\
\hline 358 & 100.0 & 100.0 & 0.0 \\
359 & 29.8 & 30.0 & 0.2 \\
360 & 11.4 & 11.9 & 0.5 \\
361 & 2.2 & 2.5 & 0.3 \\
362 & 0.4 & 0.7 & 0.3 \\
363 & 0.0 & 0.1 & 0.1 \\
364 & 0.0 & 0.0 & 0.0 \\
365 & 0.0 & 0.0 & 0.0 \\
366 & 0.0 & 0.0 & 0.0 \\
367 & 0.0 & 0.0 & 0.0 \\
368 & 0.0 & 0.0 & 0.0 \\
\hline
\end{tabular}

\section{$\left[\mathrm{U}-{ }^{13} \mathrm{C}\right]-\mathrm{Glu}$}

\begin{tabular}{crrr}
\hline $\mathrm{m} / \mathrm{z}$ & theory & data & difference \\
\hline 357 & 0.0 & 0.2 & 0.2 \\
\hline 358 & 0.0 & 0.0 & 0.0 \\
359 & 0.0 & 0.0 & 0.0 \\
360 & 0.0 & 0.1 & 0.1 \\
361 & 0.0 & 2.9 & 2.9 \\
362 & 5.2 & 6.6 & 1.4 \\
363 & 100.0 & 100.0 & 0.0 \\
364 & 24.5 & 25.4 & 0.9 \\
365 & 9.9 & 10.9 & 1.0 \\
366 & 1.6 & 1.4 & -0.2 \\
367 & 0.3 & 0.2 & -0.1 \\
368 & 0.0 & 0.0 & 0.0 \\
\hline
\end{tabular}

This fragment should not be used for flux analysis. M-1 is too high (1.8 mol\%). Fragment Glu- 432 is preferred. $\left[5-{ }^{13} \mathrm{C}\right]$-glutamine contains a peak at 359 but not at 358 , thus this fragment contains carbon 5. 

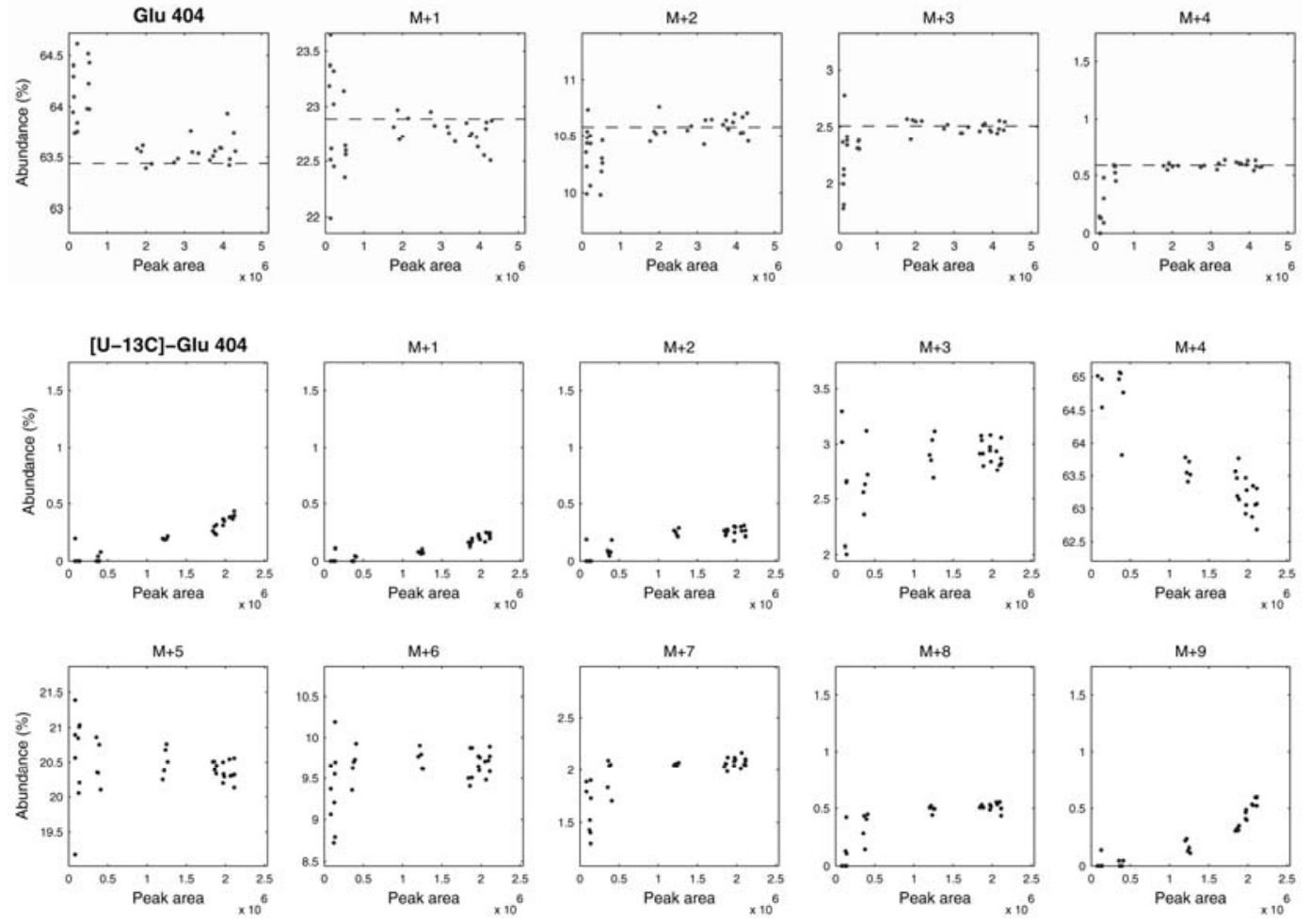

Glu 404

\begin{tabular}{ll}
\hline Formula : & $\mathrm{C}_{18} \mathrm{H}_{42} \mathrm{O}_{3} \mathrm{NSi}_{3}$ \\
Exact mass : & 404.247 \\
C-atoms : & $2-3-4-5$ \\
\hline
\end{tabular}

\begin{tabular}{crrr} 
C-atoms : & \multicolumn{3}{c}{$2-3-4-5$} \\
\hline $\mathrm{m} / \mathrm{z}$ & theory & data & difference \\
\hline 403 & 0.0 & 0.0 & 0.0 \\
\hline 404 & 100.0 & 100.0 & 0.0 \\
405 & 36.1 & 35.8 & -0.3 \\
406 & 16.7 & 16.4 & -0.3 \\
407 & 4.0 & 3.9 & -0.1 \\
408 & 0.9 & 0.8 & -0.1 \\
409 & 0.2 & 1.3 & 1.1 \\
410 & 0.0 & 0.3 & 0.3 \\
411 & 0.0 & 0.0 & 0.0 \\
412 & 0.0 & 0.0 & 0.0 \\
413 & 0.0 & 0.0 & 0.0 \\
\hline
\end{tabular}

\begin{tabular}{rrrr}
\hline $\mathrm{m} / \mathrm{z}$ & theory & data & difference \\
\hline 403 & 0.0 & 0.0 & 0.0 \\
\hline 404 & 0.0 & 0.0 & 0.0 \\
405 & 0.0 & 0.0 & 0.0 \\
406 & 0.0 & 0.1 & 0.1 \\
407 & 4.1 & 3.8 & -0.3 \\
408 & 100.0 & 100.0 & 0.0 \\
409 & 31.9 & 31.7 & -0.2 \\
410 & 15.2 & 14.6 & -0.6 \\
411 & 3.3 & 2.7 & -0.6 \\
412 & 0.8 & 0.5 & -0.3 \\
413 & 0.1 & 0.0 & -0.1 \\
\hline
\end{tabular}

This fragment can be used for flux analysis. Note that this fragment is very noisy at low concentrations, and thus should be measured at higher concentrations only. $\left[5-{ }^{13} \mathrm{C}\right]$-glutamine contains a peak at 405 but not at 404, thus this fragment contains carbon 5 . 

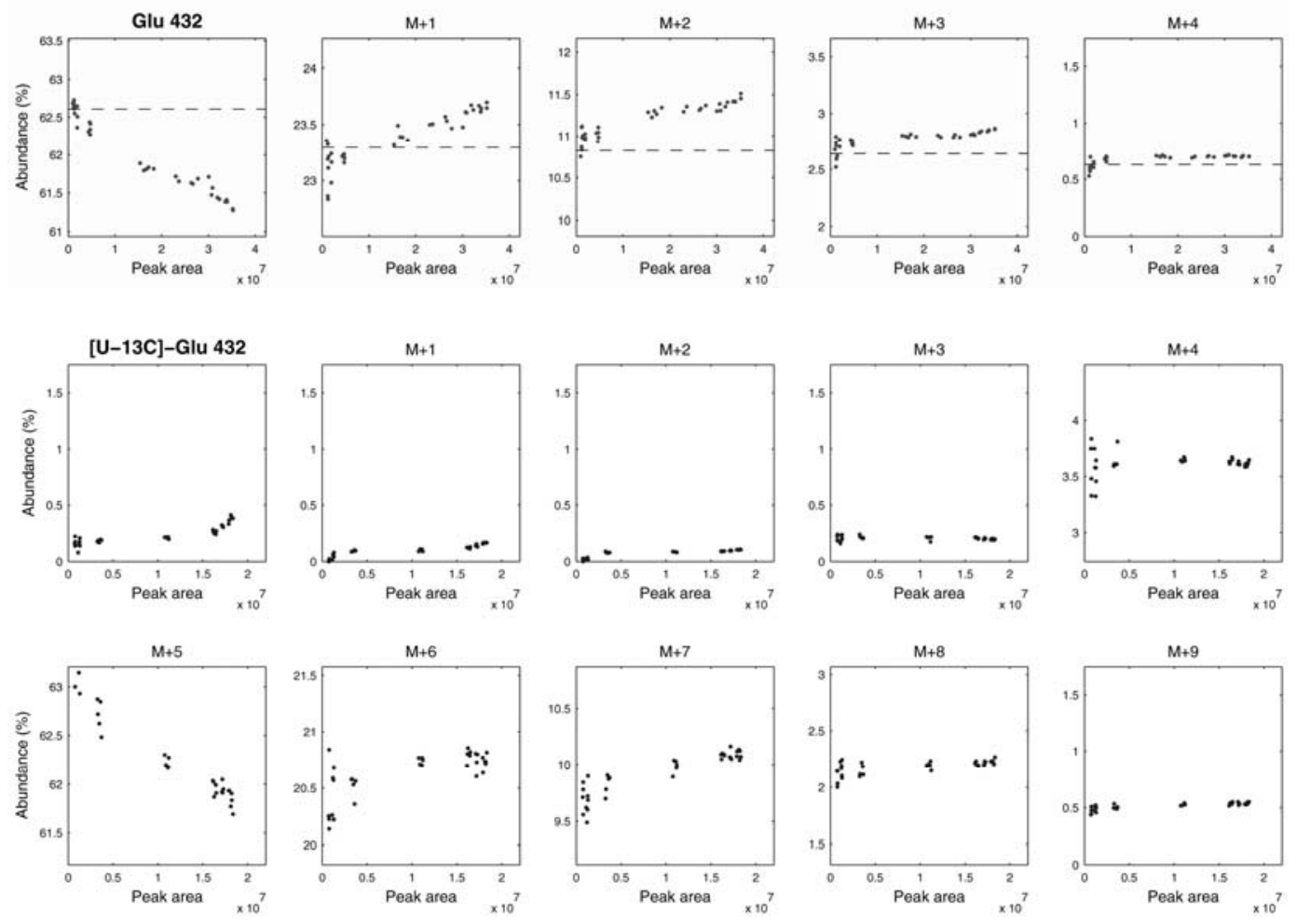

Glu 432

\begin{tabular}{lrrr}
\hline Formula : & \multicolumn{3}{c}{$\mathrm{C}_{19} \mathrm{H}_{42} \mathrm{O}_{4} \mathrm{NSi}_{3}$} \\
Exact mass : & \multicolumn{3}{c}{432.242} \\
C-atoms : & & $1-2-3-4-5$ & \\
\hline $\mathrm{m} / \mathrm{z}$ & theory & data & difference \\
\hline 431 & 0.0 & 0.0 & 0.0 \\
\hline 432 & 100.0 & 100.0 & 0.0 \\
433 & 37.2 & 37.1 & -0.1 \\
434 & 17.3 & 17.5 & 0.2 \\
435 & 4.2 & 4.4 & 0.2 \\
436 & 1.0 & 1.1 & 0.1 \\
437 & 0.2 & 0.2 & 0.0 \\
438 & 0.0 & 0.0 & 0.0 \\
439 & 0.0 & 0.0 & 0.0 \\
440 & 0.0 & 0.0 & 0.0 \\
441 & 0.0 & 0.0 & 0.0 \\
442 & 0.0 & 0.0 & 0.0 \\
443 & 0.0 & 0.0 & 0.0 \\
\hline
\end{tabular}

$\left[\mathrm{U}-{ }^{13} \mathrm{C}\right]-\mathrm{Glu}$

This fragment can be used for flux analysis. $\left[5-{ }^{13} \mathrm{C}\right]$-glutamine contains a peak at 433 but not at 432 , thus this fragment contains carbon 5 . 


\section{Lysine (Lys)}
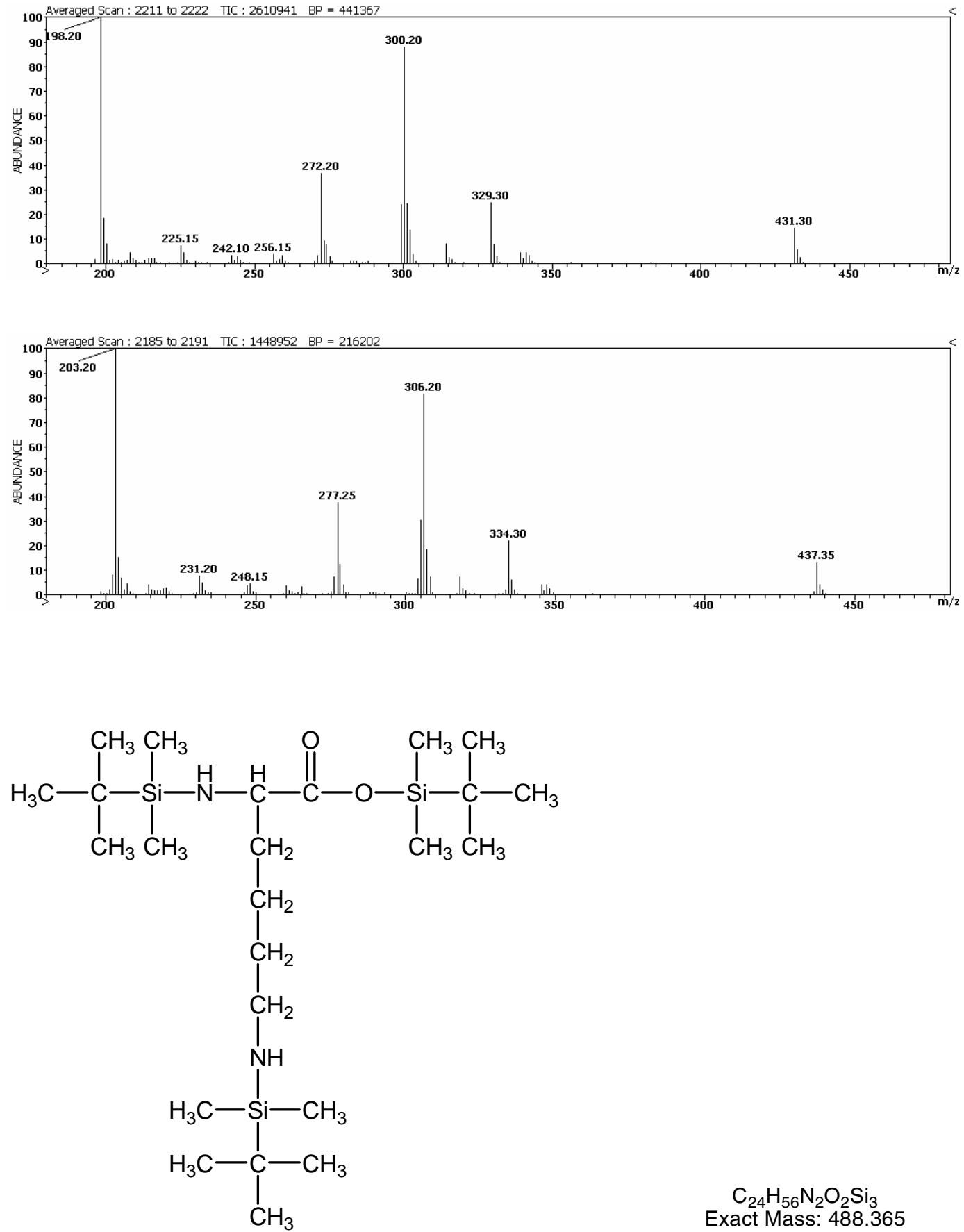

$$
\mathrm{C}_{24} \mathrm{H}_{56} \mathrm{~N}_{2} \mathrm{O}_{2} \mathrm{Si}_{3}
$$

Exact Mass: 488.365 

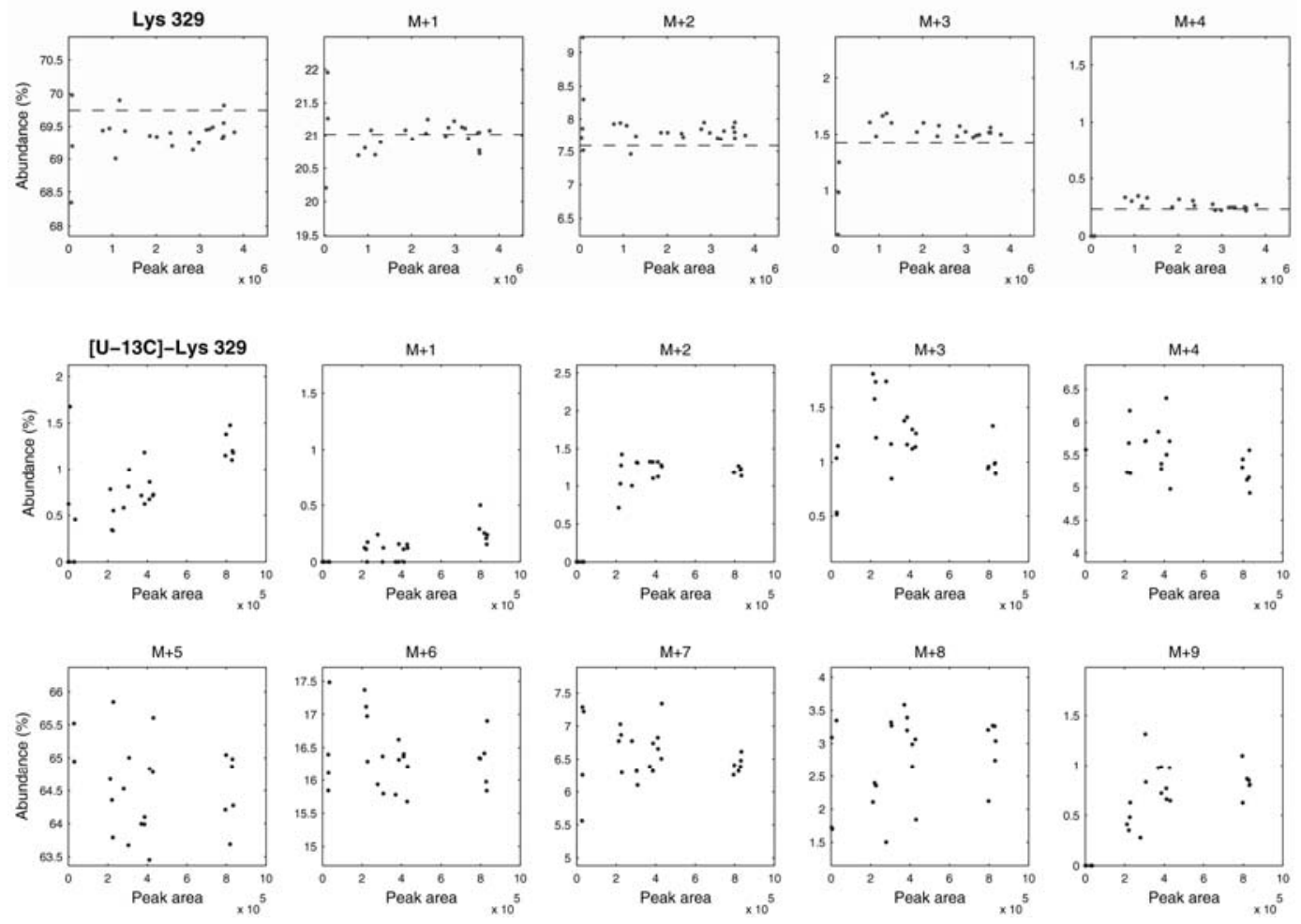

Lys 329

\begin{tabular}{lrrr}
\hline Formula : & \multicolumn{3}{c}{$\mathrm{C}_{17} \mathrm{H}_{41} \mathrm{~N}_{2} \mathrm{Si}_{2}$} \\
Exact mass : & & 329.2808 & \\
C-atoms : & & $2-3-4-5-6$ & \\
\hline $\mathrm{m} / \mathrm{z}$ & theory & data & difference \\
\hline 328 & 0.0 & 0.6 & 0.6 \\
\hline 329 & 100.0 & 100.0 & 0.0 \\
330 & 30.1 & 30.1 & 0.1 \\
331 & 10.9 & 11.2 & 0.3 \\
332 & 2.0 & 2.3 & 0.3 \\
333 & 0.3 & 0.5 & 0.2 \\
334 & 0.0 & 0.0 & 0.0 \\
335 & 0.0 & 0.0 & 0.0 \\
336 & 0.0 & 0.0 & 0.0 \\
337 & 0.0 & 0.6 & 0.6 \\
338 & 0.0 & 0.2 & 0.2 \\
\hline
\end{tabular}

$\left[{ }^{[}{ }^{13} \mathrm{C}\right]-\mathrm{Lys}$

\begin{tabular}{rrrr}
\hline $\mathrm{m} / \mathrm{z}$ & theory & data & difference \\
\hline 328 & 0.0 & 0.0 & 0.0 \\
\hline 329 & 0.0 & 0.5 & 0.5 \\
330 & 0.0 & 0.1 & 0.1 \\
331 & 0.0 & 1.8 & 1.8 \\
332 & 0.0 & 2.5 & 2.5 \\
333 & 5.2 & 9.4 & 4.2 \\
334 & 100.0 & 100.0 & 0.0 \\
335 & 24.8 & 25.7 & 0.9 \\
336 & 9.4 & 10.5 & 1.1 \\
337 & 1.5 & 3.8 & 2.3 \\
338 & 0.2 & 0.9 & 0.7 \\
\hline
\end{tabular}

This fragment should be used with caution. Lysine peak was not very abundant and therefore noisy. 

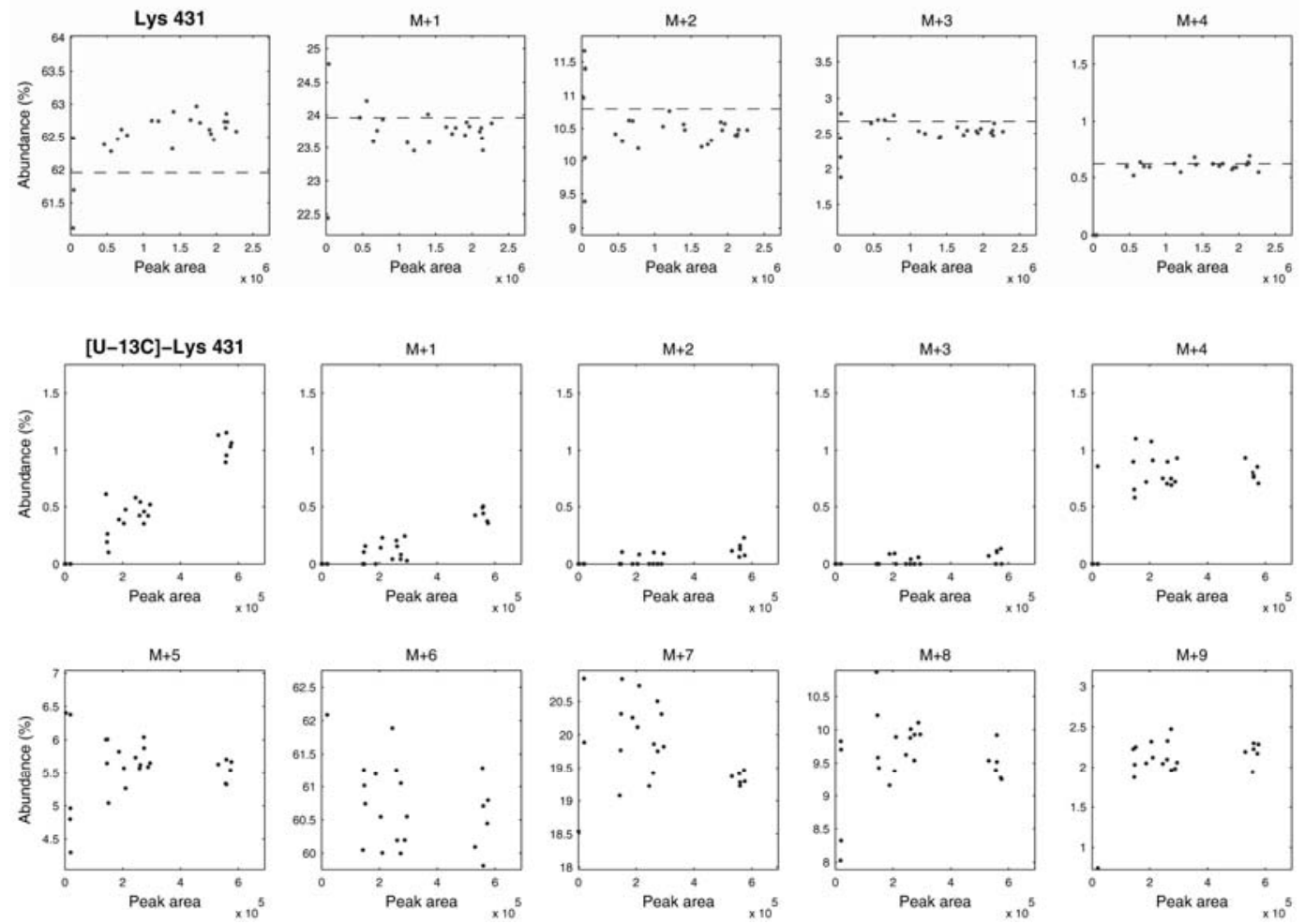

Lys 431

\begin{tabular}{lrrr}
\hline Formula : & \multicolumn{3}{c}{$\mathrm{C}_{20} \mathrm{H}_{47} \mathrm{O}_{2} \mathrm{~N}_{2} \mathrm{Si}_{3}$} \\
Exact mass : & \multicolumn{3}{c}{431.295} \\
C-atoms : & \multicolumn{3}{c}{$1-2-3-4-5-6$} \\
\hline $\mathrm{m} / \mathrm{z}$ & theory & data & difference \\
\hline 430 & 0.0 & 1.7 & 1.7 \\
\hline 431 & 100.0 & 100.0 & 0.0 \\
432 & 38.7 & 38.3 & -0.4 \\
433 & 17.4 & 16.8 & -0.6 \\
434 & 4.3 & 4.3 & 0.0 \\
435 & 1.0 & 1.0 & 0.0 \\
436 & 0.2 & 0.0 & -0.2 \\
437 & 0.0 & 0.0 & 0.0 \\
438 & 0.0 & 0.0 & 0.0 \\
439 & 0.0 & 0.0 & 0.0 \\
440 & 0.0 & 0.1 & 0.1 \\
441 & 0.0 & 0.0 & 0.0 \\
442 & 0.0 & 0.0 & 0.0 \\
443 & 0.0 & 0.0 & 0.0 \\
\hline
\end{tabular}

$\left[\mathrm{U}-{ }^{13} \mathrm{C}\right]-\mathrm{Lys}$

This fragment should not be used for flux analysis. M-1 is too high (1.7 mol\%). Lysine peak is not very abundant and therefore noisy. 


\section{Tyrosine (Tyr)}
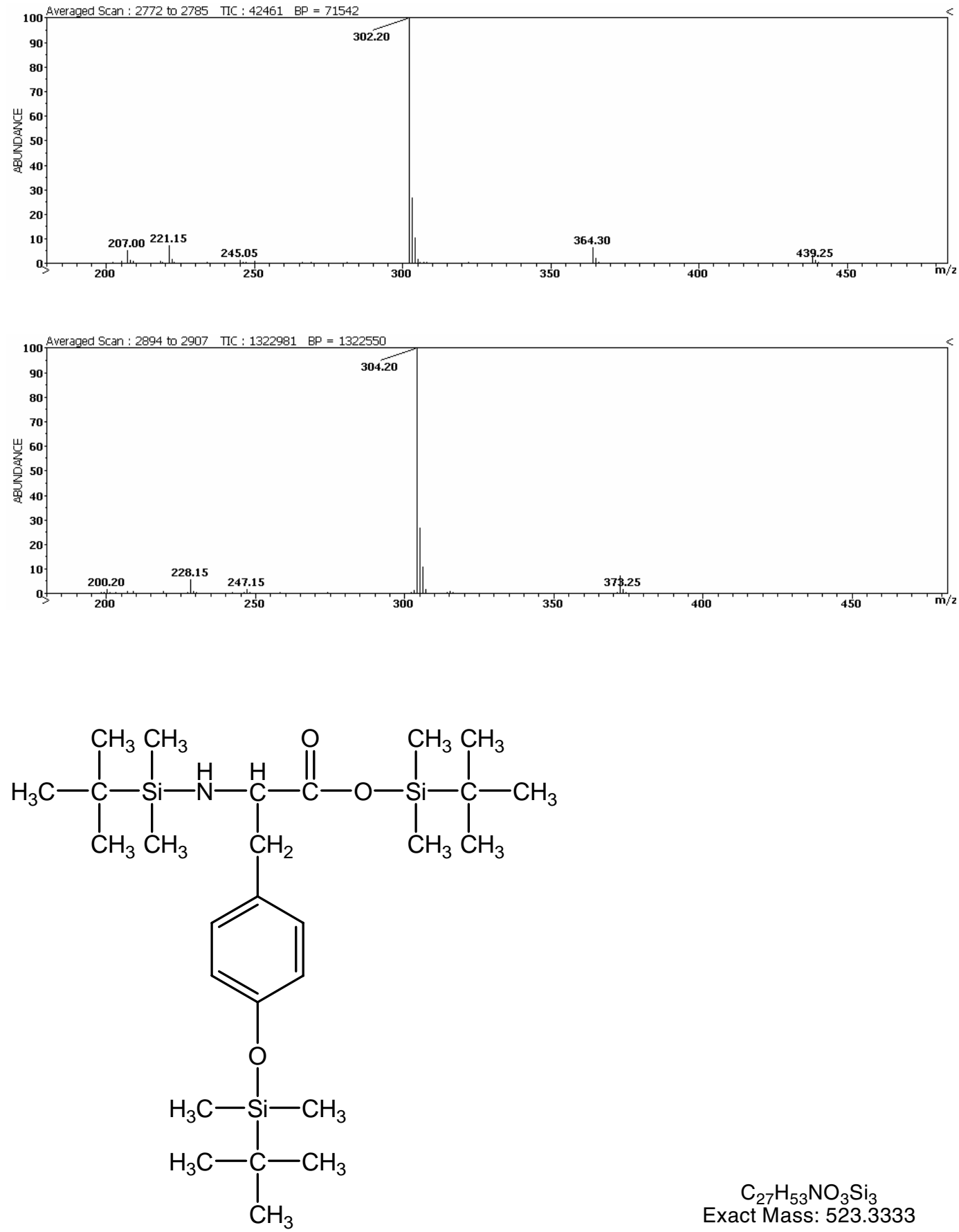

$$
\mathrm{C}_{27} \mathrm{H}_{53} \mathrm{NO}_{3} \mathrm{Si}_{3}
$$

Exact Mass: 523.3333 

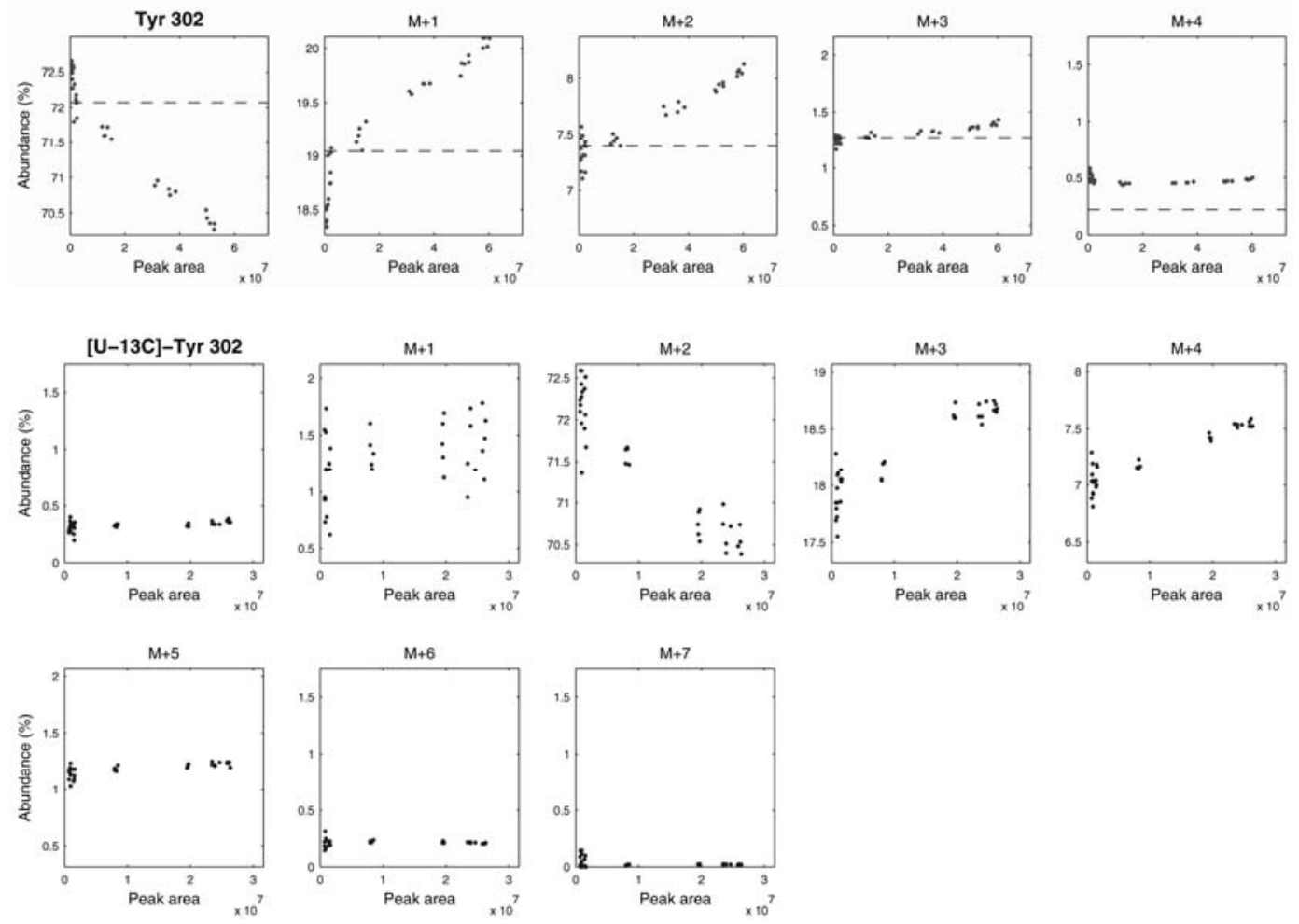

Tyr 302

\begin{tabular}{lrrr}
\hline Formula : & \multicolumn{3}{c}{$\mathrm{C}_{14} \mathrm{H}_{32} \mathrm{O}_{2} \mathrm{NSi}_{2}$} \\
Exact mass : & \multicolumn{3}{c}{302.197} \\
C-atoms : & \multicolumn{3}{c}{$1-2$} \\
\hline $\mathrm{m} / \mathrm{z}$ & theory & data & difference \\
\hline 301 & 0.0 & 0.0 & 0.0 \\
\hline 302 & 100.0 & 100.0 & 0.0 \\
303 & 26.4 & 26.3 & -0.1 \\
304 & 10.3 & 10.1 & -0.2 \\
305 & 1.8 & 1.7 & -0.1 \\
306 & 0.3 & 0.6 & 0.3 \\
307 & 0.0 & 0.8 & 0.8 \\
308 & 0.0 & 0.5 & 0.5 \\
309 & 0.0 & 0.2 & 0.2 \\
\hline
\end{tabular}

$\left[{ }^{-13} \mathrm{C}\right]-\mathrm{Tyr}$

This fragment can be used for flux analysis. Mass isotopomer distributions should be obtained from the mass range $\mathrm{m} / \mathrm{z}$ 302-305. 

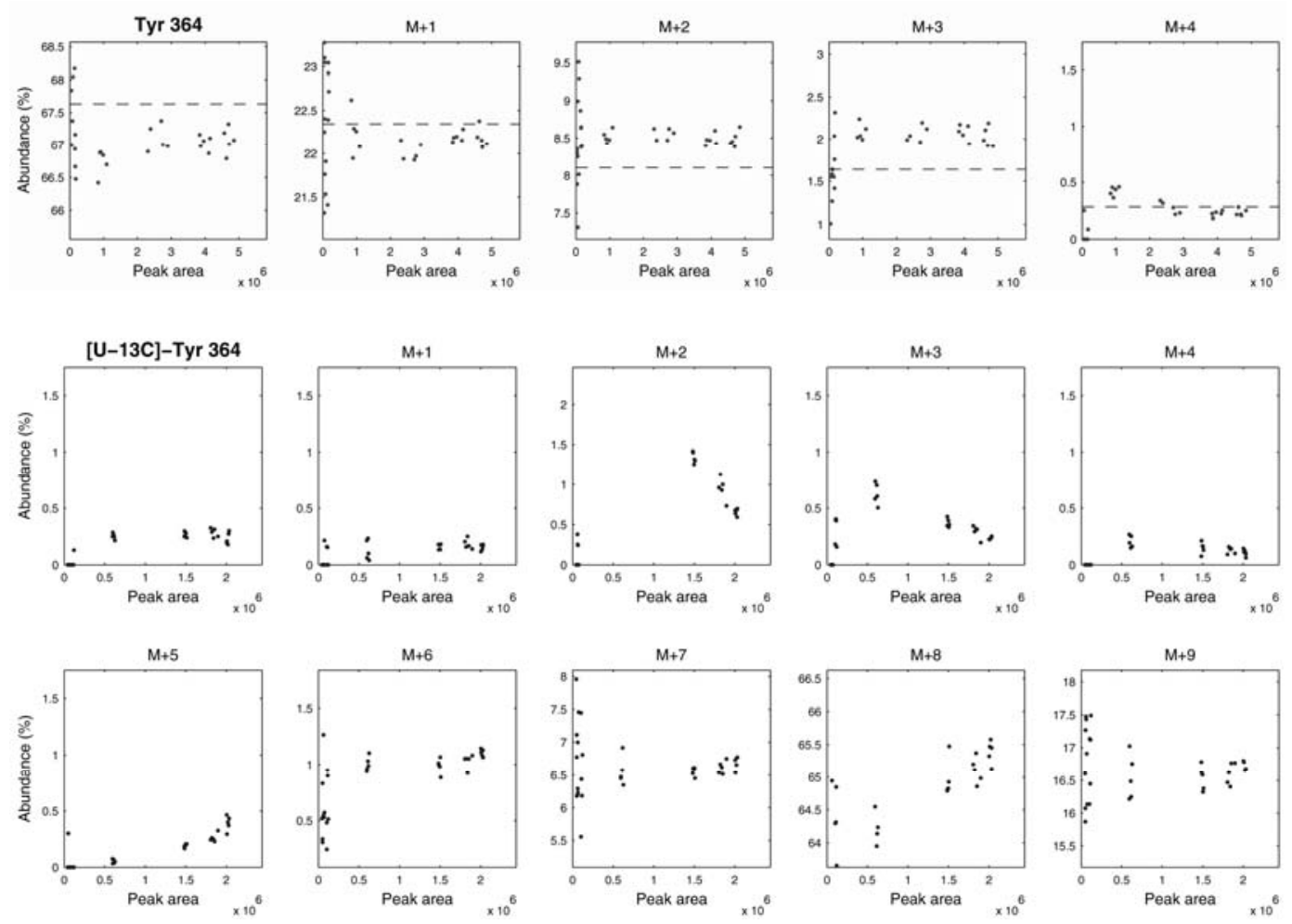

Tyr 364

\begin{tabular}{lrrr}
\hline $\begin{array}{l}\text { Formula : } \\
\text { Exact mass : }\end{array}$ & \multicolumn{3}{c}{$\mathrm{C}_{20} \mathrm{H}_{38} \mathrm{NOSi}_{2}$} \\
$\mathrm{C}$-atoms : & \multicolumn{3}{c}{ 2-3-4-5-6-7-8 } \\
\hline $\mathrm{m} / \mathrm{z}$ & theory & data & difference \\
\hline 363 & 0.0 & 0.5 & 0.5 \\
\hline 364 & 100.0 & 100.0 & 0.0 \\
365 & 33.0 & 33.1 & 0.1 \\
366 & 12.0 & 12.7 & 0.7 \\
367 & 2.4 & 3.0 & 0.6 \\
368 & 0.4 & 0.7 & 0.3 \\
369 & 0.1 & 0.1 & 0.0 \\
370 & 0.0 & 0.0 & 0.0 \\
371 & 0.0 & 0.0 & 0.0 \\
372 & 0.0 & 0.0 & 0.0 \\
373 & 0.0 & 0.0 & 0.0 \\
374 & 0.0 & 0.2 & 0.2 \\
375 & 0.0 & 0.0 & 0.0 \\
376 & 0.0 & 0.8 & 0.8 \\
377 & 0.0 & 0.3 & 0.3 \\
\hline
\end{tabular}

$\left[\mathrm{U}-{ }^{13} \mathrm{C}\right]-\mathrm{Tyr}$

This fragment should not be used for flux analysis. It is not very abundant and therefore noisy and inaccurate. Fragment Phe-308 is preferred. 


\section{Histidine (His)}

Histidine fragments were not investigated in detail because of low signal-to-noise ratio. Moreover, histidine was not consistently found in all samples.
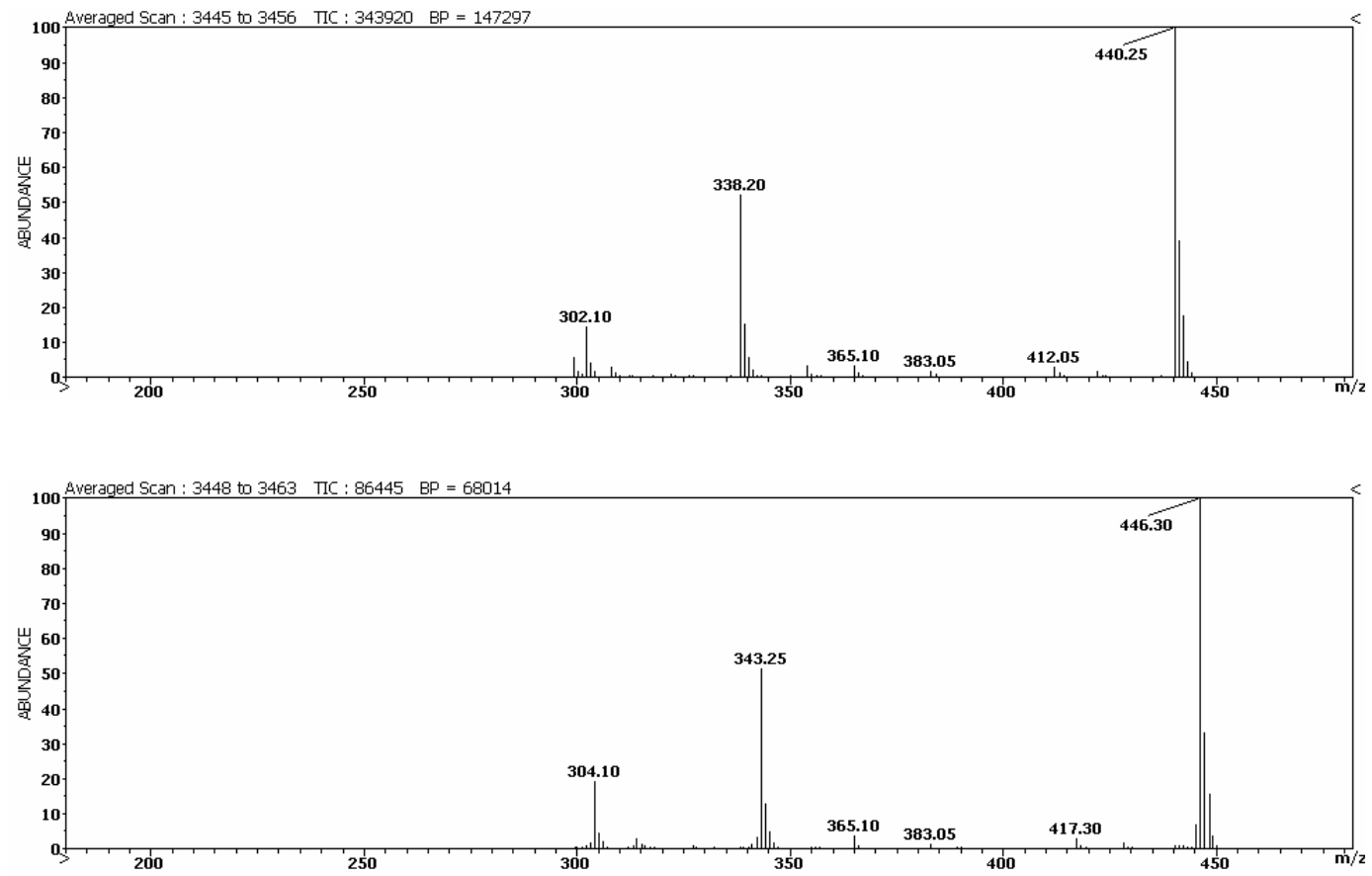<smiles>CC(C)(C)[Si](C)(C)NC(Cc1cncn1[Si](C)(C)C(C)(C)C)C(=O)O[Si](C)(C)C(C)(C)C</smiles> 


\section{Arginine (Arg)}
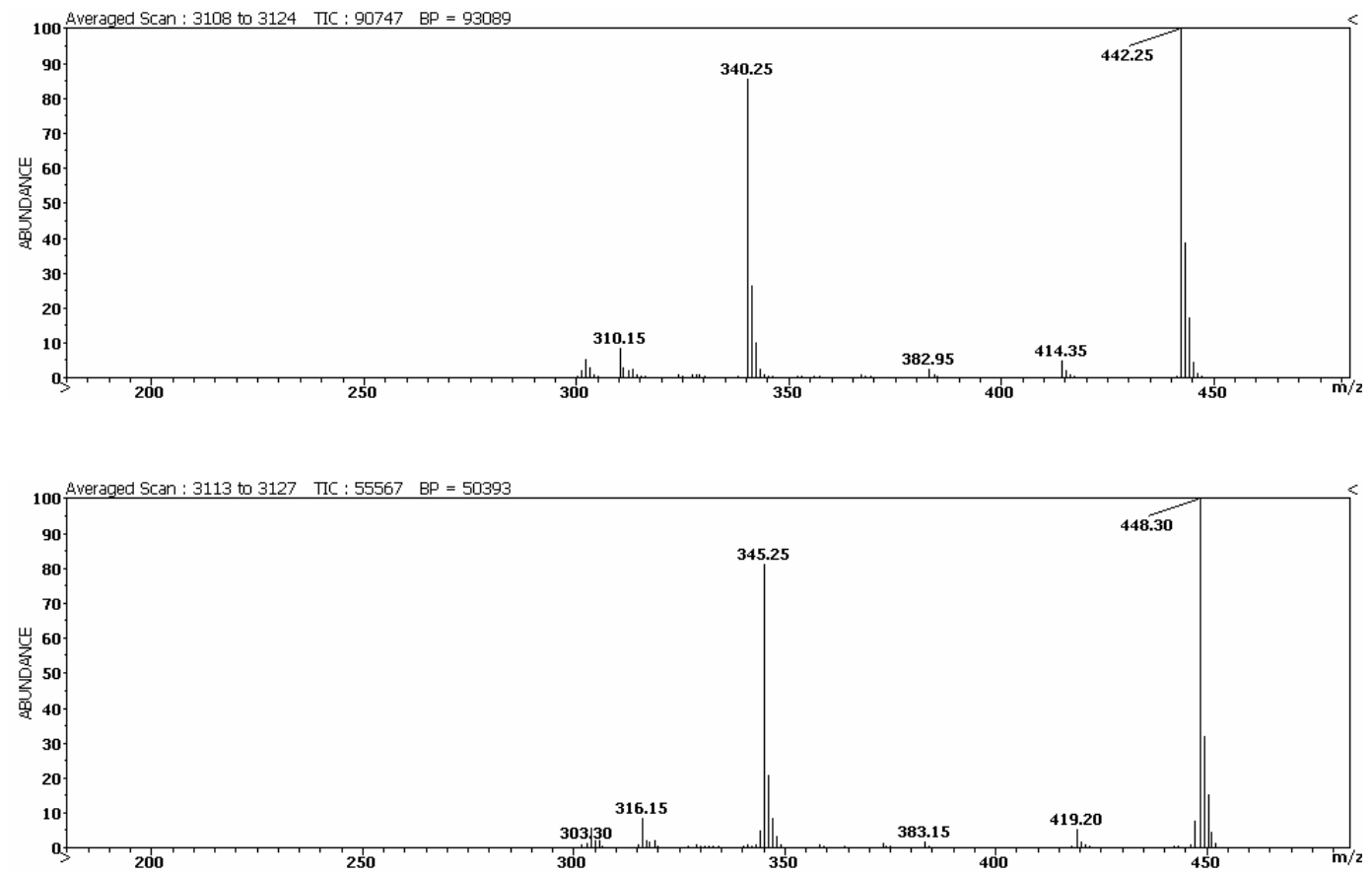<smiles>CC(C)(C)[Si](C)(C)NC(CCCN)C(=O)O[Si](C)(C)C(C)(C)C</smiles><smiles>CC(=N)N[Si](C)(C)C(C)(C)C</smiles>

Arginine peak was found at 22.5 min with peaks at m/z 442 [M-57-16], and m/z 340 [M159-16]. The arginine derivative corresponded to a nitrile form derived from loss of a guanidino nitrogen (see Petterson BW et al., Biol Mass Spectrom, 22(9), 518-523). Arginine fragments were not investigated in detail because of low signal-to-noise ratio. 